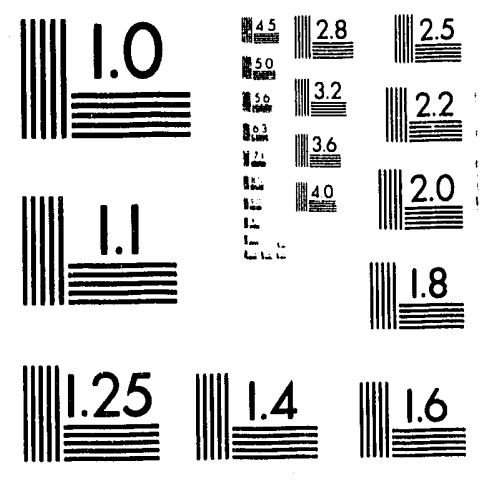



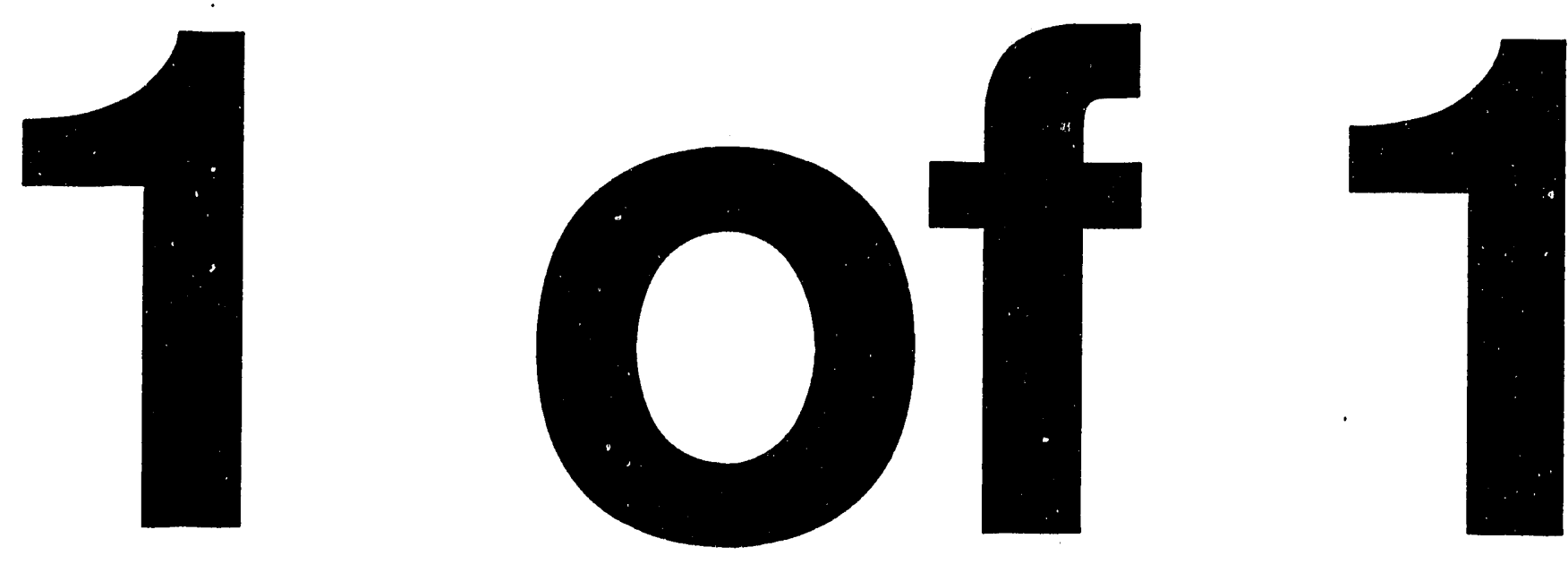


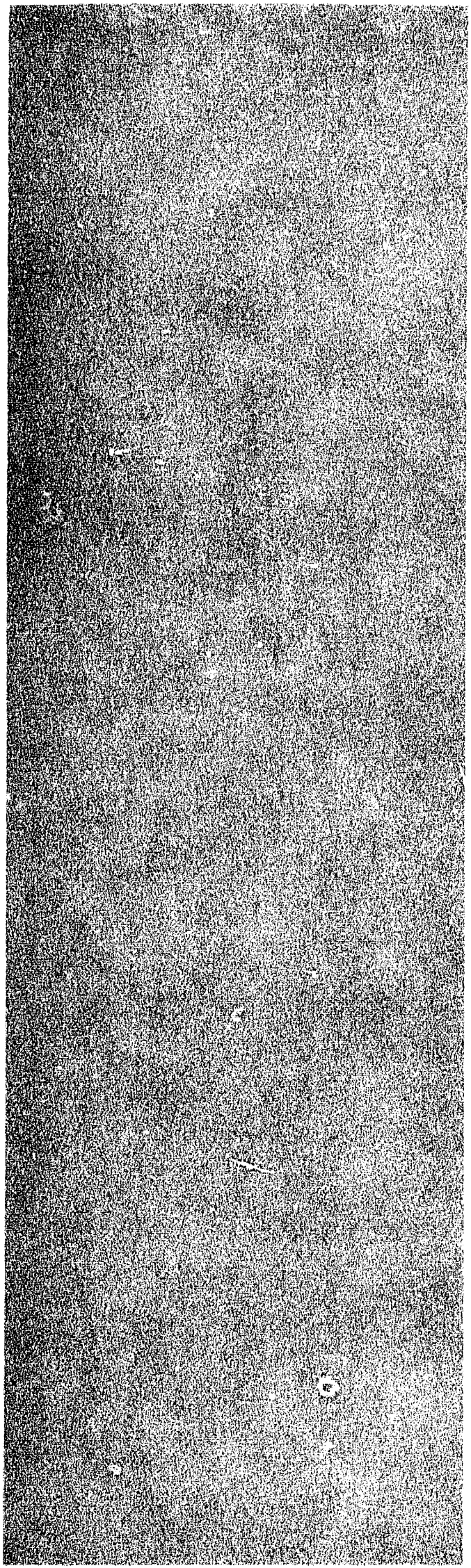

\author{
Kinetic Theory \\ of Runaway Air Breakdown \\ and the Implications for Lightning Initiation
}

\section{Los Alamos}

Los Alamos National Laboratory is operated by the University of California for the United States Department of Energy under contract W-7405-ENG-36. 


\section{(1)}

This work wass supported by the Office of Naval Research, contract \#N0001490K2010 and the US Department of Energy.

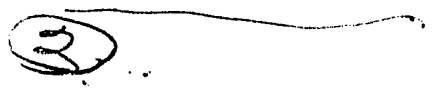

An Affirmative Action/Equal Opportunity Employer

This report was pripared as an account of work sponsored by an agency of the

United States Government. Neither The Regents of the University of California, the

United States Government nor any agency thereof, nor any of their employees, makes any warranty, express or implied, or assumes any legal liability or responsibility for the accuracy, completeness, or usefulness of any information, apparatus, product, or process disclosed, or represents that its use would not infringe privately owned rights. Reference herein to any specific commercial product, process, or service by trade name, trademark, manufacturer, or otherwise, does not necessarily constitute or imply its endorsement, recommendation, or favoring by The Regents of the University of California, the United States Government, or any agency thereof. The views and opinions of authors expressed herein do not necessarily state or reflect those of The Regents of the University of California, the United States Government, or any agency thereof. 
UC-910 and UC-000

Issued: November 1993

Kinetic Theory

of Runaway Air Breakdown

and the Implications for Lightning Initiation

R. A. Roussel-Dupré

A. V. Gurevich*

T. Tunnell**

G. M. Milikh***

- Official Visitor at Los Alamos. P.N. Lebedev Institute of Physics,

** EGEG Energy Measurements, Inc., Los Alamos, NM 87544

*** Astronomy Department, University of Maryland, College Park, MD 29742 


\title{
KINETIC THEORY OF RUNAWAY AIR BREAKDOWN AND THE IMPLICATIONS FOR LIGHTNING INITIATION
}

\author{
by
}

R. A. Roussel-Dupré, A. V. Gurevich, T. Tunnell, and G. M. Milikh

\begin{abstract}
The kinetic theory for a new air breakdown mechanism advanced in a previous paper is developed. The relevant form of the Boltzmann equation is derived and the particle orbits in both velocity space and configuration space are computed. A numerical solution of the Boltzmann equation, assuming a spatially uniform electric field, is obtained and the temporal evolution of the electron velocity distribution function is described. The results of our analysis are used to estimate the magnitude of potential $x$-ray emissions from discharges in thunderstorms and are examined in the context of lightning initiation.
\end{abstract}

\section{INTRODUCTION}

In a previous paper ${ }^{1}$ (hereafter, Paper I) we described a new mechanism for air breakdown in which high-energy electrons in the hundreds of keV range are accelerated by thunderstorm electric fields to relativistic energies (approximating "runaway"), producing secondary electrons (in the tens of $\mathrm{keV}$ range) which in turn runaway in energy and create additional secondaries. This process leads to an avalanche of the high-energy electron population, resulting in the formation of an electron beam, and requires large scale lengths to develop (hundreds of meters) at atmospheric pressure. In the presence of thunderstorm electric fields the beam propagates through the atmosphere over hundreds of meters to kilometer scale lengths and ultimately deposits a significant amount of charge outside of the region where the threshold for initiation of this mechanism is exceeded. While runaway breakdown has obvious implications for lightning initiation, it is clear that this process is a fundamental, new plasma effect that could have its manifestation in many different natural phenomena. Because of the large scale lengths necessary to produce an observable effect, however, laboratory breakdown experiments have not to date identifed the presence of this mechansim. Indeed, it will be necssary to perform experiments at very high atmospheric pressures in order to study this process in the laboratory. In the absence of such experiments, it is instructive to examine runaway breakdown in the context of lightning initiation, to review the initial speculations advanced 
concerning the production of high-energy secondary electrons in thunderstorms, and to mention the recent $x$-ray measurements which clearly suggest that this mechanism is at work in thunderstorms.

The notion that secondary electrons generated by cosmic rays could be accelerated over large distances (kilometers) by thunderstorm electric fields was first proposed by Wilson 2,3 in 1924. Since that time a number of experiments have been performed to look for the presence of high-energy electrons in the electrical discharges of thunderstorms, either by detecting them directly or by measuring the corresponding bremsstrahlung radiation emitted by these electrons. The early results were conflicting. Schonland 4 (1930) found no evidence for high-energy electrons in an ionization chamber placed beneath thunderstorms while Schonland and Viljoen 5 (1933) observed a direct correlation between Geiger counter measurements and lightning flashes occuring at tens of kilometers distance. Halliday ${ }^{6}(1934)$ corroborated the results of Schonland and Viljoen using a cloud chamber synchronized to lightning flashes. In Macky's ${ }^{7}$ (1934) experiments, balloon flights of photographic plates through active thunderstorms produced no blackening attributable to high-energy electrons while in the experiments of Hill ${ }^{8}$ (1963), electron-sensitive emulsions placed on a 300 -m tower where lightning was observed to strike produced no tracks of high-energy electrons. In 1967 Shaw ${ }^{9}$ reported a general increase in count rates associated with nearby thunderstorm activity as measured by a scintillation counter fielded on Mt. Lemmon, Arizona. In 1979 Whitmire $^{10}$ attributed the enhanced radiation measured by dosimeters placed on a $500-\mathrm{m}$ tower to $\mathrm{x}$-rays produced by lightning. More recently, Parks et al. ${ }^{11}$ (1981) and McCarthy and Parks ${ }^{12}$ (1985) have flown x-ray spectrometers through thunderstorms and observed sharp increases in the $x$-ray flux from 3 to $110 \mathrm{keV}$ prior to lightning strikes. Noting that a substantial population of cosmic ray secondary electrons could be maintained by thunderstorm electric fields, McCarthy and Parks ${ }^{13}$ investigated the possibility that bremsstrahlung emission associated with these high-energy electrons could be the source of the enhanced $x$-ray flux. They concluded however, that under the most favorable conditions, this mechanism could not account for the measured level of $x$-rays. A review of this subject was provided by $D^{\prime}$ Angelo ${ }^{14}$ (1987) who also reported observing a pronounced increase in the number of electron tracks in a diffusion cloud chamber coincident with nearby lightning flashes.

In this paper the kinetic theory for the air breakdown mechanism advanced in Paper I is developed. The relevant form of the Boltzmann equation is derived in Sec. II while the particle orbits in both velocity space and configuration space are obtained in Sec. III. A numerical solution of the Boltzmann equation, assuming a spatially uniform electric field, is presented in Sec. IV. In Sec. V, the results of our analysis are used to estimate the magnitude of potential $x$ ray emissions and are examined in the context of lightning initiation. It is shown that the details of electron beam formation and charge deposition could well account for the observed $x$-ray fluxes measured by Shaw and McCarthy and Parks and at the same time provide new insight into the initiation and development of lightning strokes. Concluding remarks are provided in Sec. VI. 


\section{BOLTZMANN EQUATION}

The Boltzmann equation for the electron velocity distribution function at high-energies $(\varepsilon>10 \mathrm{keV})$ with a uniform, externally applied electric field can be written

$$
\frac{\partial \mathrm{f}}{\partial \mathrm{t}}-\left[\frac{\left(1-\mu^{2}\right)}{\mathrm{p}} \frac{\partial \mathrm{f}}{\partial \mu}+\mu \frac{\partial \mathrm{f}}{\partial \mathrm{p}}\right] \mathrm{eE}=\frac{\partial_{\mathrm{e}}}{\partial \mathrm{t}},
$$

where $f$ is the distribution function, $p$ is the magnitude of the electron momentum, $\mu$ is the cosine of the angle between the electric field and the momentum vector (see Fig. 1), $E$ is the magnitude of the applied electric field, and $\frac{\partial_{\mathrm{e}} \mathrm{f}}{\partial \mathrm{t}}$ is the Boltzmann collision integral for electron-air interactions. At high energies, electron-air interactions are primarily Coulomb in nature and the electrons undergo small deflections in both velocity space and configuration space. In this limit, the collision integral can be written

$$
\begin{aligned}
\frac{\partial \mathrm{e}^{f}}{\partial t}= & \frac{1}{\mathrm{p}^{2}} \frac{\partial\left(\mathrm{p}^{2} \mathrm{~F}_{\mathrm{D} f}\right)}{\partial \mathrm{p}}+\frac{\left(\frac{\mathrm{Z}}{2}+1\right) \mathrm{F}_{\mathrm{D}}}{4 \gamma \mathrm{p}} \frac{\partial}{\partial \mu}\left[\left(1-\mu^{2}\right) \frac{\partial \mathrm{f}}{\partial \mu}\right] \\
& +\frac{\mathrm{N}_{\mathrm{m}} \beta \mathrm{c}}{1+(\varepsilon / \alpha)^{2}} \frac{1}{\pi \alpha} \int_{\varepsilon_{\mathrm{L}}}^{\infty} \mathrm{d} \varepsilon^{\prime} \sigma_{\mathrm{tot}}\left(\varepsilon^{\prime}\right)\left(\frac{\gamma^{2}-1}{\gamma^{2}-1}\right)\left[\mathrm{f}\left(\varepsilon^{\prime},-\left(1-\mu^{2}\right)^{1 / 2}\right)+\mathrm{f}\left(\varepsilon^{\prime},\left(1-\mu^{2}\right)^{1 / 2}\right)\right],
\end{aligned}
$$

where $F_{D}$ is the dynamical friction force first obtained by Bethe ${ }^{15}$; namely,

$$
\begin{aligned}
\mathrm{F}_{\mathrm{D}} & =\frac{4 \pi \mathrm{Z \textrm {Z } ^ { 4 } \mathrm { N }} \mathrm{m}}{\mathrm{mc}^{2}} \frac{\gamma^{2}}{\gamma^{2}-1} \\
& -\left\{\ln \left[\frac{\mathrm{mc}^{2}\left(\gamma^{2}-1\right)^{1 / 2}(\gamma-1)^{1 / 2}}{\sqrt{2 I}}\right]-\left(\frac{2}{\gamma}-\frac{1}{\gamma^{2}}\right) \frac{\ln 2}{2}+\frac{1}{2 \gamma^{2}}+\frac{(\gamma-1)^{2}}{16 \gamma^{2}}\right\},
\end{aligned}
$$

$\mathrm{Z}(=14.5)$ is the mean molecular charge for air, $\mathrm{I}=80.5 \mathrm{eV}$ for air, $\mathrm{e}$ is the electron charge, $\mathrm{c}$ is the speed of light, $m$ is the electron mass, $N_{m}$ is the molecular density, $\gamma=\frac{1}{\sqrt{1-\beta^{2}}}, \beta=\frac{v}{c}, v$ is the electron speed, $\varepsilon\left[=(\gamma-1) \mathrm{mc}^{2}\right]$ is the electron kinetic energy, $\alpha=13.85 \mathrm{eV}$, and where we have integrated the doubly differential cross-section for ionization, $\sigma\left(\varepsilon^{\prime}, \varepsilon, \xi\right)$, over the scattering angles assuming that the secondary electron (of energy $\varepsilon$ ) is emitted at $90^{\circ}$ to the incident electron (of energy $\varepsilon^{\prime}$ ) as discussed in Paper I (see also Bethe and Ashkin ${ }^{16}$ ). A plot of the dynamical friction force (normalized to its minimum value) as a function of energy $\varepsilon$ is provided in Fig. 2. Note that a minimum exists at $\varepsilon_{\min }=1.4 \mathrm{MeV}$ corresponding to $\gamma_{\min } \approx 3.8$. 
The doubly differential ionization cross-section is given by 17.19

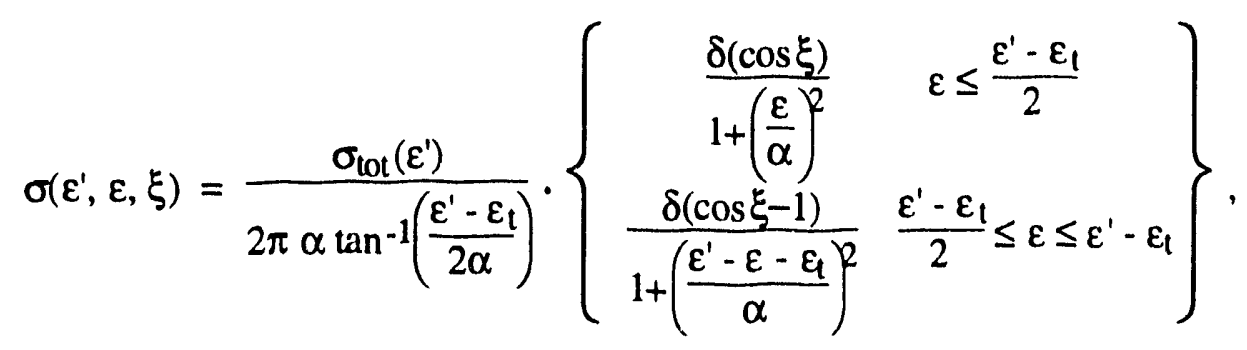

with $\varepsilon^{\prime}$ equal to the energy of the incident electron, $\mu^{\prime}$ equal to the cosine of the angle between the incident electron momentum and the electric field, $\varepsilon_{f}(=15 \mathrm{eV})$ equal to the threshold energy for ionization, $\varepsilon$ equal to the energy of the scattered electron, $\xi$ equal to the angle between the incident electron and the scattered primary electron (see Fig. 3 for a sketch of the collision geometry), and where $\sigma_{t o t}\left(\varepsilon^{\prime}\right)$ is defined as

$$
\sigma_{\mathrm{tot}}\left(\varepsilon^{\prime}\right)=\frac{\pi \mathrm{Z}^{2} \mathrm{aa}}{\mathrm{mc}^{2}} \frac{\gamma^{\prime 2}}{\gamma^{\prime 2}-1} \mathrm{z}_{1}\left\{8.68+\ln \left(\gamma^{\prime 2}-1\right)+\frac{1}{\gamma^{\prime 2}}\right\}
$$

where $a_{0}$ is the Bohr radius and $z_{1}$ is a constant of order unity with the value $z_{1}=0.877$ for air. A plot of the total ionization cross-section as a function of $\varepsilon$ is presented in Fig. 4 . Note that a minimum exists at $\varepsilon_{\min } \approx 1 \mathrm{MeV}$. While Eq. (4) for the doubly differential cross-section is applicable over the entire energy range of secondary electron production, a more accurate representation at high energies $\left(\varepsilon>1 \mathrm{keV}\right.$ ) was derived by Moller ${ }^{20}$ assuming that the dominant ionization mechanism involves Coulomb interactions between pairs of free electrons. In the case of collisions with air molecules the electron can be considered free if it is ejected with an energy that is large compared to its binding energy, which is the situation of interest in our analysis. Moller's result can be written (see Bethe and Ashkin ${ }^{16}$ )

$$
\sigma_{M}\left(\varepsilon^{\prime}, \varepsilon, \xi\right)=\frac{2 \pi Z e^{4}}{m c^{2}} \frac{\gamma^{2}}{\gamma^{2}-1}\left[\frac{1}{\varepsilon^{2}}-\frac{1}{\varepsilon\left(\varepsilon^{\prime}-\varepsilon\right)} \frac{\left(2 \varepsilon^{\prime}+m c^{2}\right) m c^{2}}{\left(\varepsilon^{\prime}+m c^{2}\right)^{2}}+\frac{1}{\left(\varepsilon^{\prime}-\varepsilon\right)^{2}}+\frac{1}{\left(\varepsilon^{\prime}+m c^{2}\right)^{2}}\right] \frac{\delta(\cos \xi)}{2 \pi}
$$


Eq. (6) is valid only for secondary electrons produced within the approximate range $\varepsilon_{\min } \leq \varepsilon \leq \frac{\varepsilon^{\prime}-\varepsilon_{\mathrm{t}}}{2}$ with $\varepsilon_{\min } \approx 1 \mathrm{keV}$. The angular dependence appended to Eq. (6) assumes only small deflections of the incident electron. Both forms of the differential ionization cross-sections were used in our calculations and only minor quantitative differences were noted in the final results.

In order to better comprehend the origin of the right-hand side of Eq. (2), it is necessary to elaborate on the derivation. The general form of the collision integral (not given here) is composed of two parts. One is a source term that accounts for those collisions that populate a small volume element in velocity space around $\varepsilon$ and $\mu$, while the second corresponds to a sink term that accounts for collisions that depopulate this region. The net result (sum of source and sink terms) of small deflections caused hy elastic, inelastic, and ionizing collisions is contained in the first two terms on the right-hand side of Eq. (2) while the production of secondary electrons is contained in the third term. We note that the former terms are consistent with a Fokker-Planck treatment where energy diffusion in velocity space is neglected but angular diffusion is included. The Fokker-Planck equation itself is derivable from the full Boltzmann collision integral (e.g., see Chapman and Cowling ${ }^{21}$ ) in the limit of small deflections. The first term on the right-hand side of Eq. (2) considers energy loss resulting from interactions with the electrons surrounding air atoms while the second term considers angle scattering resulting from electron and nuclear interactions. The detailed form of the scattering term is based on an analysis employed by Longmire and Longley 22 (and references therein) in which the effective nuclear charge seen by the fast elerirui decreases with decreasing electron energy as screening becomes more important. In the case of ionızing collisions, the dynamical friction term incorporates the effect of populating or depopulating the local region of velocity space of interest as a result of energy loss by the primary electron, assuming that it loses only a small fraction of its initial energy and experiences only small deviations from its initial trajectory. The contribution to this term from ionizing collisions therefore originates from the second half of the doubly differential ionization cross-section [Eq. (4)] combined with the loss part of the collision integral. On the other hand, the third term on the right hand side of Eq. (2) includes the contribution of ionizing collisions in which the secondary electron populates the region in velocity space of interest. The secondary electron in this context is defined to be the electron with the lower energy after collision and the appropriate ionization cross-section is given by the first half of Eq. (4) or Eq. (6). Thus, the lower limit on the integral is given by $\varepsilon_{L}=2 \varepsilon+\varepsilon_{\text {. }}$. We note that this third collision term in Eq. (2) is not generally present in a Fokker-Planck treatment and is crucial to our analysis as it accounts for the contribution of the runaway electrons to the population of low-energy electrons.

Finally, we note that the collision terms given by Eq. (2) are all proportional to the neutral density as expected for electron-air interactions. Dividing Eq. (1) through by the ncutral 
density leads to a parameterization of the solution for the electron distribution function in terms of the atmospt.eric pressure, i.e., time will scale as $\mathrm{Pt}$ and the applied field as E/P where $\mathrm{P}$ is the atmospheric pressure. The results presented helow were obtained for an altitude of $5 \mathrm{~km}\left(\mathrm{~N}_{\mathrm{m}}=\right.$ $1.32 \times 10^{19} \mathrm{~cm}^{-3}, \mathrm{P}=372$ torr) for direct comparison with thunderstorm parameters but can be scaled in this way to any altitude (moving to higher pressure will lead to shorter time scales while higher equivalent fields will be necessary to obtain the same distribution functions). This same scaling is used to describe swarm experiments.

\section{PARTICLE ORBITS}

The orbit equations are obtained by first recasting Eqs. (1) and (2) in the form

$$
\begin{aligned}
\frac{\partial f}{\partial t}- & {\left[\frac{\left(1-\mu^{2}\right)}{p} \frac{\partial f}{\partial \mu}+\mu \frac{\partial f}{\partial p}\right] e E-F_{D} \frac{\partial f}{\partial p}=\left(\frac{\partial F_{D}}{\partial p}+\frac{2 F_{D}}{p}\right) f+\frac{\left(\frac{Z}{2}+1\right) F_{D}}{4 \gamma p} \frac{\partial}{\partial \mu}\left[\left(1-\mu^{2}\right) \frac{\partial f}{\partial \mu}\right] } \\
& +\frac{N_{m} \beta c}{1+(\varepsilon / \alpha)^{2}} \frac{1}{\pi \alpha} \int_{\varepsilon_{L}}^{\infty} d \varepsilon^{\prime} \sigma_{t o t}\left(\varepsilon^{\prime}\right)\left(\frac{\gamma^{2}-1}{\gamma^{2}-1}\right)\left[f\left(\varepsilon^{\prime},-\left(1-\mu^{2}\right)^{1 / 2}\right)+f\left(\varepsilon^{\prime},\left(1-\mu^{2}\right)^{1 / 2}\right)\right] .
\end{aligned}
$$

The fourth term on the left-hand side of Eq. (7) corresponds to the effect of the dynamical friction force in slowing down high-energy electrons along their trajectory. The orbit equations are then derived by setting the total differential of the distribution function equal to the left-hand side of Eq. (7) and matching coefficients of the derivatives with the result

$$
\frac{d p}{d t}=-e E \mu-F_{D}
$$

and

$$
\frac{d \mu}{d t}=\frac{-e E\left(1-\mu^{2}\right)}{p} .
$$

Taking the ratio of Eq. (8) to Eq. (9) and expressing momentum in terms of $\gamma$ yields the equation

$$
\frac{d \gamma^{2}}{d \mu}-\frac{2 \mu}{\left(1-\mu^{2}\right)}\left(\gamma^{2}-1\right)-\frac{2 \ln \left[\frac{m c^{2} \sqrt{\left(\gamma^{2}-1\right)(\gamma-1)}}{\sqrt{2 I}}\right]}{a \delta_{0}\left(1-\mu^{2}\right)} \gamma^{2}=0 .
$$


where

$$
a \equiv \frac{\gamma_{\min }^{2}}{\gamma_{\min }^{2}-1} \ln \left[\frac{\mathrm{mc}^{2} \sqrt{\left(\gamma_{\min }^{2}-1\right)\left(\gamma_{\min }-1\right)}}{\sqrt{2}}\right] \approx 11 \text { and } \delta_{0} \equiv \frac{\mathrm{eE}}{\mathrm{F}_{\min }} \text { with } \mathrm{F}_{\min }=\frac{4 \pi \mathrm{Ze}^{4} \mathrm{~N}_{\mathrm{m}} a}{\mathrm{mc}^{2}} \text {. }
$$

In the nonrelativistic limit Eq. (10) reduces identically to Eq. (12) of Paper I with $\delta_{0}$ and $a$ defined in the same way.

The threshold electric field needed to maintain a population of high-energy electrons and initiate the avalanche process is defined to be $E_{t}=\frac{F_{\min }}{e}$. Normalizing out the molecular density (or air pressure $P$ ) we have $\frac{E_{\mathrm{t}}}{\mathrm{P}}=2.87 \mathrm{~V} \mathrm{~cm}^{-1}$ torr $^{-1}$. For comparison, the threshold electric field for air breakdown caused by the acceleration of thermal electrons with initial energy $\approx .03 \mathrm{eV}$ is $\frac{E_{\mathrm{q}}^{*}}{\mathrm{P}}=30 \mathrm{~V} \mathrm{~cm}^{-1} \mathrm{torr}^{-1}$, a factor of ten greater.

In the low-energy limit, i.e., $\gamma<\gamma_{\min }$, the natural $\log$ term is assumed to be a constant over the energy range of interest and it is then possible to obtain an analytic form for the solution to Eq. (10); namely,

$$
\gamma^{2}-1=\frac{\left(\gamma_{i}^{2}-1\right)\left(1-\mu_{i}^{2}\right)\left[\frac{1+\mu_{i}}{1-\mu_{i}}\right]^{\eta}+2 \eta \int_{\mu_{i}}^{\mu}\left[\frac{1+\mu^{\prime}}{1-\mu^{\prime}}\right]^{\eta} d \mu^{\prime}}{\left(1-\mu^{2}\right)\left[\frac{1+\mu}{1-\mu}\right]^{\eta}}
$$

where $\gamma_{i}$ and $\mu_{i}$ are the initial $\gamma$ and $\mu$ values and $\eta=\frac{\ln \left[\frac{m c^{2} \sqrt{\left(\gamma_{i}^{2}-1\right)\left(\gamma_{i}-1\right)}}{\sqrt{2 I}}\right]}{a \delta_{0}}$.

In the high-energy limit, it is not possible to assume that the natural log term is a constant. Nevertheless, an analytic form can be found for the solution to Eq. (10) assuming $\gamma \gg 1$. The result can be written 


$$
\gamma^{2}-1=\frac{1}{b} \exp \left(\frac{1+\mu}{1-\mu}\right)^{\eta_{1}}\left\{\begin{array}{c}
\left(\frac{1-\mu_{i}}{1+\mu_{i}}\right)^{\eta_{1}}\left[\ln \left[b\left(\gamma_{i}^{2}-1\right)\right]+\frac{\mu_{i}}{\eta_{1}}\right]-\frac{1}{\eta_{1}}\left(\frac{1-\mu}{1+\mu}\right)^{\eta_{1}} \mu \\
+\frac{1}{\eta_{1}} \int_{\mu_{i}}^{\mu}\left(\frac{1-\mu^{\prime}}{1+\mu^{\prime}}\right)^{\eta_{1}} d \mu^{\prime}
\end{array}\right\} .
$$

where $b=\left(\frac{\mathrm{mc}^{2}}{\sqrt{2 I}}\right)^{\frac{4}{3}}$ and $\eta_{1}=\frac{3}{4 a \delta_{0}}$.

The equivalent relativistic separatrix or "runaway line" as defined by Eq. (13) in Paper I for the non-relativistic case is obtained by calculating the values of $\gamma_{i}$ and $\mu_{i}$ from Eq. (11) for which $\gamma>\gamma_{\min }$ and $\mu=-1$. We find

$$
\mu_{s}=1-2\left[\frac{\eta}{1-\eta} \frac{1}{\gamma_{s}^{2}-1}\right]^{\frac{1}{1+\eta}}
$$

where $\eta$ was defined previously. The importance of the separatrix is that it separates velocity space into two regimes. Those electrons injected at an angle $\theta_{\mathrm{s}}$ to the applied electric field with an energy greater than $\varepsilon_{\mathrm{s}}\left[=\left(\gamma_{\mathrm{s}}-1\right) \mathrm{mc}^{2}\right]$ possess trajectories that take them ultimately to infinite energies while those with energies below $\varepsilon_{s}$ possess trajectories leading to zero energy. A plot of $\frac{\varepsilon_{\mathrm{s}}}{\mathrm{mc}^{2}}$ vs $\mu_{\mathrm{s}}$ for $\delta_{0}=2$ is provided in Fig. 5. A direct comparison with Fig. 2 of Paper I (which has an ordinate equal to $\frac{2 \varepsilon_{\mathrm{s}}}{\mathrm{mc}^{2}}$ in the nonrelativistic regime) indicates that relativistic effects for $\delta_{0}$ $\leq 2$ are important. In the vicinity of $\mu_{s}=0$, the threshold energy for runaway for $\delta_{0}=2$ is larger by a factor of approximately 1.35 compared with the results obtained in Paper I. Plots of $\frac{\varepsilon_{s}}{\mathrm{mc}^{2}}$ vs $\delta_{0}$ for $\mu_{s}=0$ and $\mu_{s}=-1$ are given in Figs. 6(a) and 6(b), respectively. The former plot identifies the minimum energy necessary for electrons injected perpendicular to the electric field to runaway in energy. These electrons in principle play an important role in defining the avalanche rate as is shown in Paper I because they are produced by the primary population of runaway electrons moving as a beam antiparallel to the electric field and because they ultimately accelerate into the direction of the beam and thereby add to the beam population. The detailed kinetic results presented below indicate, however, that transport across the separatrix is important and that it is the minimum runaway energy associated with those electrons that are moving antiparallel to the electric field [see Fig. $6(\mathrm{~b})$ ] that, for example, defines the ionization rate. 
To further illustrate certain aspects of runaway and particle collimation in configuration space, we have computed electron trajectories in both velocity space and configuration space. Plots of $\gamma-1$ as a function of $\mu$ for two initial energies corresponding to $\varepsilon_{i}=0.05$ and $0.5 \mathrm{MeV}$ $\left(\gamma_{i}=1.10\right.$ and 1.98, respectively) and five initial $\mu$-values corresponding to $\mu_{i}=-0.8,-0.4,0.0$, 0.4 , and 0.8 are shown in Figs. 7(a)-7(b) with $\delta_{0}=2.0$. Time increases along the trajectories in these plots from positive $\mu$-values to negative $\mu$-values and the calculations span a maximum time of approximately $2 \mu \mathrm{s}$. For those particles with initial energy below the separatrix $\left(\varepsilon_{\mathrm{s}}=110 \mathrm{keV}\right.$ for $\mu=-1, \delta_{0}=2$ ) we find that the orbits move toward $\mu=-1$ but that the electrons simply lose energy monotonically. For those electrons with initial energy greater than $\varepsilon_{s}$ and moving counter to the field, the orbits are such that they accelerate and eventually turn into a direction approaching $\mu=-1$. Those electrons moving along the field decelerate initially; however, if their energy is sufficiently high these electrons eventually turn into a direction counter to the field while at lower energies they simply continue to lose energy and leave the energy regime of interest.

The temporal variation of $\gamma$ and $\mu$ along the particle trajectories can be found simply by integrating Eq. (9). Plots of $\gamma-1$ vs $t$ and $\mu$ vs $t$ for the same initial energies and $\mu$-values noted above are shown in Figs. 8(a)-8(b) and 9(a)-9(b) respectively. For initial energies below the threshold $(<110 \mathrm{keV})$ and all corresponding initial $\mu$-values we see that the particle energies decrease monotonically with time. Above the threshold ( $>110 \mathrm{keV}$ ) some or all of the orbits show increasing energy as a function of time. The plots of $\mu$ vs $t$ are straightforward to interpret.

Assuming that the applied field points along the $\mathrm{z}$-direction of a cylindrical coordinate system in configuration space, the electron trajectories can be computed from the results obtained above and the additional equations

$$
\begin{aligned}
& \frac{\mathrm{d} z}{\mathrm{dt}}=\sqrt{\frac{\gamma^{2}-1}{\gamma^{2}} \mu \mathrm{c}} \text { and } \\
& \frac{\mathrm{d} \rho}{\mathrm{dt}}=\sqrt{\frac{\left(\gamma^{2}-1\right)\left(1-\mu^{2}\right)}{\gamma^{2}}} \mathrm{c} .
\end{aligned}
$$

Plots of particle trajectories for an initial energy corresponding to $\varepsilon_{\mathrm{i}}=0.5 \mathrm{MeV}$ and five initial $\mu$-values corresponding to $\mu_{\mathrm{i}}=-0.8,-0.4,0.0,0.4$, and 0.8 are shown in Fig. 10 with $\delta_{0}=2.0$. We find that for this initial energy and $\mu \leq 0.0$. he particles turn rapidly into the direction of the electric field, i.e. within $9 \mathrm{~m}$ or $30 \mathrm{~ns}$ [e.g., see Fig. 9(b)]. Note that particles with initial energy less than $\varepsilon_{s}=110 \mathrm{keV}$ are effectively stationary and their trajectories are not plotted. 


\section{SOLUTION OF BOLTZMANN EQUATION}

\section{A. Numerical technique}

The temporal evolution of the electron distribution is obtained by solving Eq. (7) numerically on a two dimensional mesh of $\ln (\varepsilon)$ and $\mu$. Our numerical treatment evolves around a Taylor series expansion of the distribution function in time written as

$$
f(t+\Delta t, \gamma, \mu)=f(t, \gamma, \mu)+\frac{\partial f}{\partial t} \Delta t+\frac{\partial^{2} f}{\partial t^{2}} \frac{\Delta t^{2}}{2}+\cdots
$$

The Boltzmann equation defines a time derivative operator $L$ which allows us to rewrite Eq. (16) as

$$
f(t+\Delta t, \gamma, \mu)=f(t, \gamma, \mu)+L(f) \Delta t+L[L(f)] \frac{\Delta t^{2}}{2}+\cdots
$$

where

$$
\begin{aligned}
L(f) \equiv & {\left[\frac{\left(1-\mu^{2}\right)}{\sqrt{\gamma^{2}-1}} \frac{\partial \mathrm{f}}{\partial \mu}+\mu \frac{\sqrt{\gamma^{2}-1}}{\gamma} \frac{m c^{2}}{\varepsilon} \frac{\partial \mathrm{f}}{\partial \ln (\varepsilon)}\right] \frac{\mathrm{eE}}{\mathrm{mc}}+\frac{\sqrt{\gamma^{2}-1}}{\gamma} \frac{\mathrm{F}_{\mathrm{D}}}{\mathrm{mc}} \frac{\mathrm{mc} \mathrm{c}^{2}}{\varepsilon} \frac{\partial \mathrm{f}}{\partial \ln (\varepsilon)} } \\
& +\left(\frac{\sqrt{\gamma^{2}-1}}{\gamma \mathrm{mc}} \frac{\mathrm{mc}}{\varepsilon} \frac{\partial \mathrm{F}_{\mathrm{D}}}{\partial \ln (\varepsilon)}+\frac{2 \mathrm{~F}_{\mathrm{D}}}{\mathrm{mc} \sqrt{\gamma^{2}-1}}\right) \mathrm{f}+\frac{\left(\frac{\mathrm{Z}}{2}+1\right) \mathrm{F}_{\mathrm{D}}}{4 \mathrm{mc} \sqrt{\gamma^{2}-1}} \frac{\partial}{\partial \mu}\left[\left(1-\mu^{2}\right) \frac{\partial \mathrm{f}}{\partial \mu}\right] \\
& +\frac{\mathrm{N}_{\mathrm{m}} \beta \mathrm{c}}{\left\{1+[\varepsilon / \alpha]^{2}\right\}} \frac{1}{\pi \alpha} \int_{\ln \left(\varepsilon_{\mathrm{D}}\right)}^{\infty} \mathrm{dln}\left(\varepsilon^{\prime}\right) \varepsilon^{\prime} \sigma_{\mathrm{tot}}\left(\varepsilon^{\prime}\right)\left(\frac{\gamma^{2}-1}{\gamma^{2}-1}\right)\left[\mathrm{f}\left(\varepsilon^{\prime},-\left(1-\mu^{2}\right)^{1 / 2}\right)+\mathrm{f}\left(\varepsilon^{\prime},\left(1-\mu^{2}\right)^{1 / 2}\right)\right],
\end{aligned}
$$

and where we have made use of the fact that $\mathrm{L}$ is independent of time. Although the operator $\mathrm{L}$ in Eq. (18) is defined in terms of the ionization differential cross-section given by Eqs. (4) and (5), we have also performed calculations with $\sigma_{M}$ given by Eq. (6). A fourth order finite difference approximation is used to evaluate the $\varepsilon$ - and $\mu$-derivatives while a trapezoidal approximation is used for the integral. Although the Taylor series expansion can be easily taken to any order, our calculations are generally carried to order two. Given an appropriate velocity- 
space mesh that is capable of resolving the dynamical friction force and the ionization crosssection, the time step $\Delta \mathrm{t}$ is then chosen in such a way as to insure that the Courant condition is satisfied over the entire grid. We note that this numerical technique for solving the Boltzmann equation has been used previously in the modeling of swarm experiments.

The energy of the lower boundary $\left[\varepsilon_{\mathrm{LB}}=\left(\gamma_{\mathrm{B}}-1\right) \mathrm{mc}^{2}\right]$ is chosen to be greater than or equal to $10 \mathrm{keV}$, so that the approximations inherent to the derivation of the dynamical friction force are valid, and less than the lower energy at which the dynamical friction force equals the force exerted by the applied field. The value at the upper boundary $\left[\varepsilon_{U B}=\left(\gamma_{U B}-1\right) \mathrm{mc}^{2}\right]$ is chosen large enough for the value of the distribution function to fall off to zero. The distribution function at the lower energy boundary is found by solving the Boltzmann equation in the same way as outlined above except that the $\varepsilon$-derivative is set equal to the derivative calculated at the midpoint between the boundary point and the next energy value. A similar technique is used to calculate the distribution function at $\mu=1$ and -1 , for all $\varepsilon$. These boundary conditions permit particles to flow by deceleration (acceleration) below (above) the lower (upper) energy boundary and for particles to be produced at the lower boundary by ionization.

\section{B. Results}

Results were obtained for three cases corresponding to three values of the parameter $\delta_{0}\left(\equiv \mathrm{E} / \mathrm{E}_{\mathrm{t}}\right)=2.0,5.0$, and 8.0. The relevant computational parameters used in our calculations for all cases are listed in Table I. The initial distribution function was taken in each case to be isotropic and a Gaussian in energy with a mean energy of $\varepsilon_{\mathrm{m}}=2 \mathrm{MeV}$ and a full-width at half maximum (FWHM) $\varepsilon_{\mathrm{w}}=277 \mathrm{keV}$. The results presented in this paper were obtained with the differential ionization cross-section $\sigma_{M}$ given by Eq. (6). Three-dimensional plots of the distribution function as a function of $\log \varepsilon$ and $\mu$ are shown for six different times in Figs. 11(a)-11(f) for $\delta_{0}=2$. After an initial transient phase (first three plots) the distribution function settles into a self-similar solution (last three plots) where the shape remains approximately constant while the number density continues to grow. The shape of the distribution function at late times is determined effectively by the competition between the electric field force and the dynamical friction and by the production of secondary electrons. Above the threshold energy $\left[\varepsilon_{s}(-1,2)=110 \mathrm{keV}\right]$ electrons accelerate and avalanche. Just below the threshold electrons are decelerating out of that energy range faster than they are produced by ionization and there is a decrease in the electron population. At still lower energies the secondary production rate dominates and the distribution function increases in magnitude. The peak in the distribution function at a $90^{\circ}$ angle to the electric field at low energies $\left[\varepsilon<\varepsilon_{\mathrm{S}}(-1,2)\right]$ is caused by the fact that the avalanching electrons are confined to move along the electric field and are ejecting secondary electrons at $90^{\circ}$ to their direction of motion. 
TABLE I. Computational parameters.

\begin{tabular}{|c|c|c|c|}
\hline Case & $\delta_{0}=2.0$ & $\delta_{0}=5.0$ & $\delta_{0}=8.0$ \\
\hline Altitude $(\mathrm{km})$ & 5.0 & 5.0 & 5.0 \\
$t_{\max }(\mathrm{ns})$ & 150 & 75.0 & 37.5 \\
$\Delta \mathrm{t}(\mathrm{ns})$ & 0.00005 & 0.00005 & 0.00002 \\
$\varepsilon_{\min }(\mathrm{MeV})$ & 0.01 & 0.01 & 0.005 \\
$\varepsilon_{\max }(\mathrm{MeV})$ & 50 & 50 & 100 \\
$\Delta \ln (\varepsilon)$ & 0.08517 & 0.08517 & 0.09903 \\
$\Delta \mu$ & 0.033 & 0.033 & 0.033 \\
\hline
\end{tabular}

A unique population of electrons which we refer to as the "beam" is carved out of the distribution function by requiring that their energies lie above the instantaneous threshold defined by

$$
-\mathrm{eE} \mu \geq \mathrm{FD}_{\mathrm{D}} .
$$

Electrons with energy above this threshold are accelerating and turning into the direction antiparallel to the applied electric field. Scattering has the effect of smoothing out or diffusing the sharp boundaries defined by this condition. Plots of the beam distribution obtained in this way are provided in Figs. 12(a)-12(f) for the same times as presented in Figs. 11(a)-11(f). A more quantitative picture is attained by plotting the beam distribution function vs energy for several values of $\mu$ [see Figs. 13(a)-13(f)]. Note that the bump on the tail of the distribution represents the remnants of the initial Maxwellian distribution function which possessed an initial mean energy of $2 \mathrm{MeV}$.

Plots of the electron density, mean kinetic energy, and energy spread obtained by taking appropriate moments of the distribution function as a function of time are given in Figs. 14(a)-14(c) for the overall distribution function and in Figs. 14(d)-(f) for the beam. The energy spread is defined to be the standard deviation of the distribution function times a factor equal to $\sqrt{\ln 2}$. For a Gaussian distribution the energy spread would be equivalent to the FWHM. From Figs. 14(a) and 14(d) we see that the electron density for both the overall distribution and the beam avalanches after an initial transient phase with an average e-folding time of $27 \mathrm{~ns}$. The mean kinetic energy for the overall distribution [Fig. 14(b)] decays from the initial 2-MeV value to a nearly steady-state value of approximately $0.61 \mathrm{MeV}$. Similarly, the mean energy of the beam reaches a nearly constant value of $0.95 \mathrm{MeV}$. The energy spread for both distributions 
[Figs. 14(c) and 14(f)] increases from the initial value of $277 \mathrm{keV}$ to maximum values of 1.75 $\mathrm{MeV}$ (overall) and $2.05 \mathrm{MeV}$ (beam) and subsequently decreases, as the population of lowenergy secondaries continues to rise, reaching steady-state values of $1.18 \mathrm{MeV}$ (overall) and $1.45 \mathrm{MeV}$ (beam). We note that the energy moments reported here for the overall distribution function are dependent on the energy chosen for the lower boundary. If the range of our calculations was extended to lower energies, we would obtain lower mean energies for the overall distribution function. A summary of the steady-state moments discussed above is provided in Table II.

TABLE II. Steady-state moments of overall and beam distribution functions.

\begin{tabular}{|c|c|c|c|}
\hline Case & $\delta_{0}=2.0$ & $\delta_{0}=5.0$ & $\delta_{0}=8.0$ \\
\hline & Overall & Distribution & \\
\hline $\mathrm{t}_{\mathrm{i}}(\mathrm{ns})$ & 27 & 7.0 & 2.9 \\
$\varepsilon_{\mathrm{m}}(\mathrm{MeV})$ & 0.61 & 1.0 & 0.68 \\
$\varepsilon_{\mathrm{w}}(\mathrm{MeV})$ & 1.18 & 1.8 & 1.3 \\
\hline \multicolumn{2}{|c|}{} & Beam & Distribution \\
\hline $\mathrm{t}_{\mathrm{i}}(\mathrm{ns})$ & 27 & 7.0 & 2.9 \\
$\varepsilon_{\mathrm{m}}(\mathrm{MeV})$ & 0.95 & 1.2 & 0.82 \\
$\varepsilon_{\mathrm{w}}(\mathrm{MeV})$ & 1.45 & 1.9 & 1.4 \\
$\mathrm{~K}$ & 7.4 & 8.6 & 8.6 \\
\hline
\end{tabular}

Similar results were obtained for $\delta_{0}=5$ and 8 . Plots for these cases are not provided; however, a summary of the steady-state results is presented in Table II. The behavior of the distribution function with increasing field strength is tied strongly to the corresponding decrease in threshold energy for beam formation. The latter values for $\delta_{0}=5$ and 8 are $\varepsilon_{\mathrm{s}}\left(-1, \delta_{0}\right)=30.0$ and $16.2 \mathrm{keV}$, respectively. As the threshold energy decreases the beam electrons occupy a larger regime in phase space (both in angle and energy) and the ionization rate increases because the secondary production rate varies as $1 / \varepsilon^{2}$ [cf. Eq. (2)]. For the range of field strengths discussed in this paper, $t_{i}$ is proportional to $\xi_{\xi}^{x}\left(-1, \delta_{0}\right)$ where $x$ is approximately 1.2 . In the non-relativistic regime $\left(\varepsilon_{\mathrm{s}} \ll \mathrm{mc}^{2}\right.$, or $\left.\delta_{0}>2\right)$, $\mathrm{x}$ equals 1.43 . For values of $\delta_{0}$ below 1.5 the ionization rate is controlled primarily by the acceleration of electrons into and out of the phase space occupied by the electron beam and as a result the ionization rate decreases substantially relative to that predicted by the latter proportionality. When $\delta_{0}$ is increased from 2 to 5 we see an increase in both the mean energy and energy spread of the beam distribution function. This effect results not only from the fact that at higher fields the beam electrons occupy a larger energy range but also 
because the electrons can accelerate to higher energies on the same time scale as production of the low-energy population proceeds. At higher field strengths $\left(\delta_{0}=8\right)$ the production rate of lowenergy electrons proceeds at a faster rate than does acceleration to higher energy and the mean energy and energy spread of the distribution function decreases. The details of these effects can be seen in the plots of the self-similar beam distribution functions shown for the three values of $\delta_{0}$ in Figs. 15(a)-(c).

The ionization rate discussed above can be written in terms of the ionization length obtained in Paper I; namely.

$$
v_{i}\left(\delta_{0}\right)=\frac{\beta_{m} c K\left(\delta_{0}\right)}{\lambda_{\text {ion }}}:: \frac{4 \pi N_{m} z^{4} \beta_{m} K\left(\delta_{0}\right)}{m c \varepsilon_{s}\left(0, \delta_{0}\right)} \frac{\gamma_{m}^{2}}{\gamma_{m}^{2}-1},
$$

where $\varepsilon_{\mathrm{s}}\left(0, \delta_{0}\right)$ is the separatrix value of the minimum energy for runaway evaluated at $\mu_{\mathrm{s}}=0$ and a field of strength $\delta_{0}, \gamma_{\mathrm{m}}$ is the relativistic factor for an electron with the mean energy of the beam, $\beta_{\mathrm{m}}$ is the corresponding speed normalized to the speed of light, and $K\left(\delta_{0}\right)$ is a correction factor that incorporates all of the kinetic effects not included in the analysis of Paper $I$. The kinetic correction factor accounts for the details of transport in velocity space over a broad energy range, scattering of electrons across the separatrix, and the competition between acceleration of electrons in the runaway regime and the rate of production of these electrons by ionization in determining the shape of the distribution function and ultimately the net avalanche rate. Inverting Eq. (20) one can solve for $\mathrm{K}$ with the result

$$
K\left(\delta_{0}\right)=\frac{\operatorname{mc} \varepsilon_{s}\left(0, \delta_{0}\right) v_{i}\left(\delta_{0}\right)}{4 \pi N_{m} \mathrm{ze}^{4} \beta_{m}} \frac{\gamma_{m}^{2}-1}{\gamma_{m}^{2}}
$$

Given the results plotted in Fig. 6 for $\varepsilon_{s}\left(0, \delta_{0}\right)$ and the values computed for $v_{i}\left(\delta_{0}\right)$, we can calculate $\mathrm{K}\left(\delta_{0}\right)$. The results are presented in Table II and indicate that kinetic effects are extremely important, yielding a factor of eight enhancernent in the ionization rate compared to analytic estimates. The source of this enhancement can be traced to two effects. First, electrons at $90^{\circ}$ to the applied electric field and energies greater than twice the minimum value along the separatrix produce particles near the minimum value of the separatrix, antiparallel to the electric field. The latter electrons then accelerate in the field and produce additional secondary electrons at $90^{\circ}$ to the applied field and energies greater than twice their initial energy. The latter secondary electrons again feed the population of low-energy runaway electrons. Thus, the correct energy at which to evaluate the analytic form for the ionization rate is $\varepsilon_{s}\left(-1, \delta_{0}\right)$ instead of $\varepsilon_{s}\left(0, \delta_{0}\right)$. This effect alone accounts for a factor of three. Second, electrons in the energy range $2 \varepsilon_{\mathrm{S}}\left(-1, \delta_{0}\right) \leq \varepsilon \leq \varepsilon_{\mathrm{s}}\left(0, \delta_{0}\right)$ and in a sizeable angular range around $90^{\circ}$ to the electric field are just 
below the separatrix and are not part of the pool of runaway electrons but contribute to their production by direct ionization and by scattering into the runaway regime. This effect is not considered in the analytic formula and can easily account for an additional factor of two. The remaining discrepancy is left to the details of the calculations. To illustrate the validity of this interpretation we plot in Figs. 16(a)-16(c) for $\delta_{0}=2,5$, and 8 respectively, the ratio of the computed ionization rate for the overall population of electrons and separately for the runaway electrons to the analytic estimate as a function of time. We find that initially the ionization rate for the overall population of electrons is approximately equal to that predicted by the analytic form but that the runaway electrons are avalanching at an enhanced rate. This result indicates that the numerics are accurate and that the kinetic effects outlined above are operating on the runaway population. At later times, after reaching a self-similar solution, the overall population of electrons is forced to avalanche at the same rate as the runaway electrons.

\section{BREMSSTRAHLUNG EMISSION AND LIGHTNING INITIATION}

In order to calculate the $x$-ray emission from the electron beam described above and determine its role in initiating lightning strikes, it is first necessary to estimate the physical dimensions of the beam.

\section{A. Spatial dimensions}

The kinetic calculations discussed in the previous section apply to the case of a spatially uniform electric field and the results therefore describe the electron distribution function over all space. In a more realistic picture we focus on a single secondary electron produced by cosmic ray ionization and accelerating in a localized thunderstorm electric field. For spatial regions where the latter field exceeds the threshold for relativistic air breakdown, an electron beam, whose length along the field and whose diameter grow in time, is formed.

The length $L$ of the beam at time $\tau$ is estimated to be the dispersion in velocity $\Delta v$ along the field (obtained from the distribution function) times $\tau, L=\Delta v \tau$. A cartoon of this process is shown in Fig. 17(a). We start with a population of fast particles whose energy is above the threshold energy where the dynamical friction force equals the applied force. These electrons produce a population of slowly accelerating electrons with energies near the threshold energy and intermediate electrons that accelerate rapidly. By the next ionization time the intermediate electrons have accelerated to become fast electrons while additional slow electrons are produced in the spatial proximity of the fast and intermediate electrons. This process continues with time and the beam grows in size according to its dispersion in velocity and with a fairly uniform density profile along the direction of propagation. 
The diameter $\mathrm{D}$ of the beam grows in time both because of angle scattering and because the secondary electrons produced by primary ionization are ejected perpendicular to the beam direction of motion. In the latter case the secondary electrons eventually accelerate into the direction of the heam, producing tertiary electrons perpendicular to the direction of acceleration and these electrons in turn accelerate into the direction of the beam. The net result is a random walk that leads to a diffusive growth of the beam diameter with time [see Fig. 17(b)]. For this process alone, the diameter after a time $\tau$ is estimated to be $D=2 \sqrt{N_{\text {steps }}} L_{\text {step }}$, where $N_{\text {steps }}=\tau / t_{i}, L_{\text {step }}$ is the distance perpendicular to the heam for an electron of mean energy to turn in the direction of acceleration, and $t_{i}$ is an ionization time scale. The step size is estimated from the coefficient of the second term on the left-hand side of Eq. (1) to be $L_{s t e p}=\frac{m_{c}^{2} \gamma_{T} \beta_{T}{ }^{2}}{e E}$, where $\gamma_{\mathrm{T}}$ ' and $\beta_{\mathrm{T}}$ ' are determined at an energy equal to three times the beam threshold energy $\left[\varepsilon_{\mathrm{s}}\left(-1, \delta_{0}\right)\right]$.

The diffusive growth of the beam diameter resulting from angle scattering is treated in a report by Longmire and Longley. 22 Including the effects of collimation by an applied field whose magnitude is larger than the dynamical friction force, the heam diameter after a time $\tau$ can be approximated as

$$
D=2 \Delta v \tau \sqrt{\frac{\frac{Z}{2}+1}{\gamma_{m}\left(\delta_{0} F_{D \min } / F_{D}\right)-1}}
$$

where all energy dependent terms are evaluated at the mean energy of the beam. An additional effect that has not been considered in the preceeding treatment is the fact that in the process of producing secondary electrons, twice as many contribute to the growth in population of the central part of the beam as seen in Fig. 17(b). After many e-foldings the beam develops a density profile with a peak near the location of the initiating seed electron. To illustrate this effect we numerically simulated the random walk depicted in Fig. 17(b), starting with a single particle and extending the calculation to $N$ e-foldings, where $N$ was varied from 20 to 100 . The resulting density profile plotted as a function of the average step taken by the electron in an ionization time is shown in Fig. 18 for the case $N=40$. Note that for this case the FWHM is approximately 10 times the step size and that electrons have scattered out as far as 30 steps in rough agreement with Eq. (22). In general we also find that the FWHM increases as the square root of the number of e-foldings (or time). On the basis of this calculation we estimate the final beam diameter to be $D=\sqrt{\frac{t}{40 t_{i}}} 10 L_{\text {step, }}$, where the step-length is taken to be the square root of the sum of the squares of the steps resulting from angular scattering and secondary electron ejection in a single ionization time, i.e., 


$$
D=1.58 \sqrt{\left[\left(\frac{m^{2} \gamma_{T}^{\prime} \beta_{T^{\prime 2}}}{e E}\right)^{2}+\frac{\Delta v^{2} t_{i}^{2}\left(\frac{Z}{2}+1\right)}{\gamma_{m}\left(\delta_{0} F_{D \min } / F_{D}\right)-1}\right] \frac{t}{t_{i}}} .
$$

Given the kinetic results, the maximum beam dimensions can now be estimated from the above analysis by specifying a time for the duration of the beam. There are two mechanisms that lead to termination of the electron beam; namely, 1) the applied electric field falls below the threshold for the avalanche process to proceed and 2) the electrostatic force, resulting from charge accumulation as the beam density increases, becomes comparable to the applied field and causes the beam to breakup. For strong thunderstorm electric fields $\left(\delta_{0} \geq 2\right)$, the range of the fields is generally sufficient to cause 2 ) to occur before 1 ). In these cases, one can estimate the typical dimensions of the beam by calculating the time at which the radial electrostatic field becomes equal to the thunderstorm electric field. In the latter case careful consideration must also be given to the return current of cold electrons which could neutralize the electrostatic field of the beam and allow it to continue growing along the applied field. In order to incorporate this effect, however, a detailed self-consistent calculation would have to be performed. We will neglect the effect of the return current in our initial estimates and then comment on its relative importance a posteriori.

The radial Lorentz force exerted on an electron by a moving cylindrical distribution of charge of diameter $D$ and lengt' $L$ at radius $D / 2$ is approximately

$$
F_{r}=\frac{4 Q e}{D L \gamma_{m}^{2}}
$$

where $Q$ is the total accumulated charge. By equating expression (24) to the applied force, substituting our expressions for $\mathrm{D}$ and $\mathrm{L}$, and assuming that the total population of beam electrons exponentiates in time with a time scale $t_{i}$, we obtain the following expression for the time $\tau_{b}$ for the beam to breakup,

$$
\frac{\tau_{b}}{t_{i}}=\ln \left\{\frac{D L^{2} \gamma_{m}^{2} E}{4 e \beta_{m} c t_{i}}\right\} .
$$

Equation (25) can be solved iteratively for $\tau_{b}$, yielding a rough estimate for the maximum dimensions of the electron beam prior to breakup. The results are summarized in Table III for three values of the electric field, $\delta_{0}=2,5$, and 8 . Note that the breakup time ranges from 1.2-0.12 $\mu \mathrm{s}$, that the diameter ranges from 64-4.5 meters and that the length is less than $150 \mathrm{~m}$. The amount of charge accumulated prior to breakup ranges from 0.38-0.017 $\mathrm{C}$. The energetics associated with the beam at time $\tau_{b}$ is summarized in Table IV. For the three cases considered a 
total of approximately $15-0.46 \mathrm{MJ}$ is expended in creating the beam while an additional 8.7$0.097 \mathrm{MJ}$ is stored in the self-consistent electrostatic field of the beam. The latter corresponds effectively to the energy necessary to collimate the beam. The total power generated in creating the beam ranges from 13-5.0 TW. The implications of these results for lightning initiation are discussed below.

TABLE III. Spatial dimensions of electron beam.

\begin{tabular}{|c|c|c|c|}
\hline Case & $\delta_{0}=2.0$ & $\delta_{0}=5.0$ & $\delta_{0}=8.0$ \\
\hline$\tau_{b}(\mathrm{~ns})$ & 1200 & 300 & 124 \\
$\mathrm{~L}(\mathrm{~m})$ & 150 & 58.5 & 26.8 \\
$\mathrm{D}(\mathrm{m})$ & 64 & 11.5 & 4.5 \\
$\mathrm{Q}(\mathrm{C})$ & 0.38 & 0.021 & 0.017 \\
\hline
\end{tabular}

TABLE IV. Energetics of electron beam.

\begin{tabular}{|c|c|c|c|}
\hline Case & $\delta_{0}=2.0$ & $\delta_{0}=5.0$ & $\delta_{0}=8.0$ \\
\hline $\begin{array}{c}\text { Total Kinetic } \\
\text { Energy } \\
(\mathrm{J})\end{array}$ & $7.5 \times 10^{6}$ & $7.4 \times 10^{5}$ & $4.6 \times 10^{5}$ \\
$\begin{array}{c}\text { Total Frictional } \\
\text { Energy Expended } \\
(\mathrm{J})\end{array}$ & $7.5 \times 10^{6}$ & $7.4 \times 10^{5}$ & $4.6 \times 10^{5}$ \\
$\begin{array}{c}\text { Total Power } \\
(\mathrm{W})\end{array}$ & $1.3 \times 10^{13}$ & $5.0 \times 10^{12}$ & $7.4 \times 10^{12}$ \\
$\begin{array}{c}\text { Energy Stored in } \\
\text { Deposited Charge } \\
(\mathrm{J})\end{array}$ & $8.7 \times 10^{6}$ & $6.8 \times 10^{4}$ & $9.7 \times 10^{4}$ \\
\hline
\end{tabular}

In the process of relativistic air breakdown, a large number of low-energy secondary electrons are created. In air approximately $34 \mathrm{eV}$ is expended per ion pair produced.23,24 Therefore, we can estimate the total number of low-energy secondary electrons produced per primary as $r_{s}=\varepsilon_{m} / 34$, where $\varepsilon_{m}$ is the mean kinetic energy of the beam. For the case $\delta_{0}=2, \mathrm{r}_{\mathrm{s}} \approx 29000$ electrons per primary. Initially, the secondary electrons will respond to the applied electric field but as the beam charge builds up and the magnitude of the radial electric 
field of the beam approaches that of the applied field, the secondaries will produce a radial current that attempts to neutralize the beam. Given that the equilibration time for low-energy electrons is small compared to the heam ionization time, the secondary electrons will form a swarm whose kinetic properties are defined by the applied field. For $\delta_{0}=2$ $\left(E / P \approx 6 \mathrm{~V} \mathrm{~cm}^{-1}\right.$ torr $\left.^{-1}\right)$, the electron swarm has a characteristic energy $T_{e} \approx 1 \mathrm{eV}$ and a drift velocity $v_{d}=3.4 \times 10^{4} \mathrm{~m} \mathrm{~s}^{-1}$. The time required to neutralize the beam charge is approximately $\tau_{N} \approx D v_{m} t_{i} / \sqrt{2} L r_{s} v_{d}$, which yields a value of $\tau_{N}=2.3 n s$ for $\delta_{0}=2$. On the other hand, the time $\tau_{\mathrm{r}}\left(=\gamma_{\mathrm{m}}{ }^{2} \varepsilon_{\mathrm{m}} / \mathrm{eE} v_{\mathrm{m}}\right)$ for the beam electrons to respond to the radial electric field is approximately $142 \mathrm{~ns}$. On the basis of these results we would conclude that the beam is neutralized by the return current of cold electrons and that it will not break up due to the buildup of its own electrostatic charge. However, we note that our estimate of the neutralization time is optimistic particularly as the radial electric field hegins to exceed the thunderstorm field because the secondary electrons must also respond to the accumulated positive charge left behind by the beam. Clearly, our calculations are only approximate and a more detailed analysis is needed to accurately address this issue.

\section{B. Bremsstrahlung radiation}

The doubly-differential cross-section for bremsstrahlung emission by a relativistic electron moving through air was initially derived by Bethe and Heitler ${ }^{25}$ and is given by 26,27

$$
\frac{d^{2} \chi_{R}}{d v d \Omega^{\prime}}=\sigma_{B} \frac{p}{p_{0}}\left\{\phi_{1}+L \phi_{2}\right\} \frac{3}{16 \pi}\left[\frac{1}{\gamma_{0}^{2}\left(1-\beta_{0} \cos \psi\right)^{2}}\right]\left[1+\frac{\left(\cos \psi-\beta_{0}\right)^{2}}{\left(1-\beta_{0} \cos \psi\right)^{2}}\right] \text {, }
$$

with $\sigma_{\mathrm{B}}=\frac{\frac{\mathrm{Z}}{2}\left(\frac{\mathrm{Z}}{2}+1\right)}{137 \mathrm{v}}\left(\frac{\mathrm{e}^{2}}{\mathrm{mc}^{2}}\right)^{2}$.

$$
\begin{aligned}
& \phi_{1}=\frac{4}{3}-2 E_{0} \frac{p^{2}+p_{0}^{2}}{p^{2} p_{0}^{2}}+\zeta^{2}\left(\frac{\eta_{0} E}{p_{0}^{3}}+\frac{\eta E_{0}}{p^{3}}-\frac{\eta \eta_{0}}{p o p}\right), \\
& \phi_{2}=\left[\frac{8}{3} \frac{E_{0}}{p_{0} p}+\frac{(h v)^{2}}{p_{0}^{3} p^{3}}\left(E_{0}^{2} E^{2}+p_{0}^{2} p^{2}\right)+\frac{\zeta^{2} h v}{2 p_{0} p}\left(\frac{E_{0} E+p_{0}^{2}}{p_{0}^{3}} \eta_{0}-\frac{E_{0} E+p^{2}}{p^{3}} \eta+\frac{2 h v E_{0} E}{p^{2} p_{0}^{2}}\right)\right], \\
& L=2 \log \left(\frac{E_{0} E_{0}+p_{0} p-(h v)^{2}}{\zeta h v}\right),
\end{aligned}
$$




$$
\begin{aligned}
& \eta=2 \log _{\mathrm{e}}\left(\frac{E+\mathrm{p}}{\zeta}\right), \\
& \eta_{0}=2 \log _{\mathrm{e}}\left(\frac{E_{0}+\mathrm{p}_{0}}{\zeta}\right),
\end{aligned}
$$

where $v$ is the frequency of the emitted radiation, $h$ is Planck's constant, $E_{0}$ is the total energy of the incident electron with corresponding momentum $\mathrm{p}_{0}, \mathrm{E}\left(=\mathrm{E}_{0}-\mathrm{hv}\right)$ is the energy of the scattered electron with corresponding momentum $p, \zeta=m c^{2}, \beta_{0}$ and $\gamma_{0}$ are determined from $E_{0}$. and $\psi$ is the angle between the incident particle momentum and the emitted photon. The specific emissivity (number of photons radiated per unit volume per unit frequency per steradian per second) is then obtained by integrating over the electron distribution function; namely.

$$
\varepsilon_{v}=2 N_{m} \int d p p^{2} \int d \Omega f(p, \mu) \frac{d^{2} \chi_{R}}{d v d \Omega^{\prime}} \beta c
$$

With these results, the specific intensity at a distance $R$ from the source and an angle $\theta^{\prime}$ relative to the applied electric field, can then be approximated as

$$
I_{V}\left(R, \theta^{\prime}\right)=\frac{\varepsilon_{v} V_{s}}{R^{2}}
$$

where $V_{S}$ is the volume of the source region. A three-dimensional plot of the specific intensity calculated for $\delta_{0}=2$ and normalized to a distance of $1 \mathrm{~km}$ as a function of the photon energy and emission angle measured relative to the electric field direction is shown in Fig. 19(a). Twodimensional plots of the intensity vs angle for several photon energies are provided in Fig. 19(b). The relativistic effects that cause forward peaking of the radiation field are readily discemible at high photon energies (e.g., $1 \mathrm{MeV}$ ) where the electrons responsible for this emission have high energies $(>1 \mathrm{MeV}$ ) and are confined to a narrow angular range around the direction antiparallel to the electric field. At low energies the angular dependence hecomes less forward peaked in part because the electron distribution function is broader in angle with a maximum at $90^{\circ}$ to the electric field. 
The results presented in Figs. 19(a) and 19(b) were determined for a time equal to 150 ns after initiation of the kinetic calculations. Extrapolating these results to time $\tau_{b}$ $\left(=1200 \mathrm{~ns}\right.$ for $\left.\delta_{0}=2\right)$ assuming that the distribution function is self-similar and exponentiating in magnitude with an ionization time of $27 \mathrm{~ns}$ yields a multiplication factor equal to $7.75 \times 10^{16}$. The absorption scale length for $100-\mathrm{keV}$ photons in air is approximately $150 \mathrm{~m} 28$ so that the peak intensity observed at $1 \mathrm{~km}$ from the breakdown region is approximately $I_{100 \mathrm{keV}}$ (at $1 \mathrm{~km}$ ) $\approx 4.9 \times 10^{5}$ photons $/ \mathrm{cm}^{2} / \mathrm{s} / \mathrm{sr} / \mathrm{eV}$. Comparison of these results with measurements can only be made in the general context of lightning initiation as discussed below.

\section{Lightning initiation}

Given the results obtained above it is now possible to discuss the role played by the proposed relativistic breakdown mechanism in initiating lightning and other related discharge phenomena. Electrical discharges that occur in thunderstorms have the net effect of neutralizing the positive and negative charge layers formed in the process of cloud electrification. Neutralization occurs by depositing negative charge in the positive charge layers and by leaving positive charge behind in the negative charge layers. Intracloud discharges directly neutralize the dipole structure of the cloud while cloud-to-ground discharges neutralize the negative charge layers.

In the specific case of relativistic air breakdown, the following qualitative picture of the neutralization process emerges. As the negative and positive charge layers grow, the electric field in the vicinity of these layers eventually exceeds the local threshold field value. A highenergy secondary electron produced by a cosmic ray shower accelerates in this field and cascades into an electron beam that carries negative charge away from the negative charge layer either towards the positive charge layer or towards the ground. The positive charge that is left behind is partially neutralized by a "return" current of the less energetic electrons produced in this process. The return current permits the beam electrons to travel longer distances and the net result is that the positive charge is distributed along the length of the breakdown channel. When the strength of the electric field falls below threshold (along the propagation path), the beam electrons lose energy and deposit their charge in the form of negative ions that are effectively immobile over the millisecond time scales of interest. Overall, this process is more effective in neutralizing the positive charge layers simply because the negative charge is deposited locally within these layers whereas positive charge is distributed over larger distances outside of the negative charge layers. One interesting consequence of this form of charge transport is that localized negative charge layers can continue to grow producing stronger discharges that manifest themselves as steppedleaders and ultimately lead to cloud-to-ground lightning. In fact it is conceivable that neutralization of the negative charge layer may require a cloud-to-ground discharge that includes a return stroke which is very effective in transporting negative charge to the ground. 
If the neutralization process discussed above was effective it would limit the field strengths to values near the threshold. At $5 \mathrm{~km} \mathrm{E}$ is approximately $100 \mathrm{kV} / \mathrm{m}$ whereas the value at $10 \mathrm{~km}$ is $50 \mathrm{kV} / \mathrm{m}$. Thus, the negative charge layers would naturally have larger field strengths associated with them than the positive layers during the active phase of thunderstorms. Verti. 1 soundings of the electric field in thunderstorms tend to substantiate this qualitative result. ${ }^{29-31}$ Additional effects are associated with the magnitude with which the fields exceed threshold and the range of the fields. For example, to the extent that the charge configuration in a thundercloud can be treated as that of a capacitor, the range of the electric field between the charge layers in a thunderstorm is likely to be larger than that outside of the cloud. Thus, for a given field strength the electron beam will avalanche over longer distances in the cloud producing a more continuous discharge process with branching (provided electrostatic blowout of the beam occurs or additional breakdown channels are initiated by the radial field of the beam). If the field strengths are also larger in the cloud, then strong, luminous discharges that transport substantial charge can occur. The self-consistent field configuration in the cloud will undoubtedly be determined by a combination of the cloud electrification process and the details of the discharge mechanism. In general it will be necessary to derive such a model in order to make definitive statements about the details of electrical discharges in thunderclouds.

Nevertheless, given a rough model for the field configuration in a cloud and a value for the cosmic ray produced secondary electron flux, it is possible to obtain a more quantitative picture. The precise role played by relativistic air breakdown in initiating cloud-to-ground lightning and other electrical discharge phenomena occurring in thunderstorms depends to a large extent on the magnitude and range of the thunderstorm electric fields. In the regions where the electric field strength exceeds the threshold $\left(E_{l}=1(0) \mathrm{kV} / \mathrm{m}\right.$ at $\left.5 \mathrm{~km}\right)$, breakdown will proceed at a rate defined by the field strength while the density of the electron beam formed in this process will depend on the range of the field. The amount of current carried by the beam formed by a single secondary electron after cascading over a distance $\Delta s$ is given approximately by

$$
I=\frac{e^{2} t_{i} \exp \left(\frac{v_{i} \Delta s}{c}\right)}{L^{2}} .
$$

where $L$ is the length of the beam, e is the electron charge, $v_{i}$ is the ionization rate, and $c$ is the speed of light. At $5 \mathrm{~km}$ altitude, the cosmic ray produced secondary electron flux integrated for energies above

$1 \mathrm{MeV}$ is approximately $200-700$ electrons $/ \mathrm{m}^{2} / \mathrm{s}$. Assuming that a charge layer of several Coulombs occupies a spherical region in a thunderstorm $1 \mathrm{~km}$ in diameter with a field strength of magnitude $E=150 \mathrm{kV} / \mathrm{m}\left(\delta_{0}=1.5\right)$ and range $R=300 \mathrm{~m}$, the total current generated by cosmic ray induced breakdown in this region would range from 0.34-1.2 $\mathrm{A}$. If this process were $100 \%$ 
efficient in neutralizing the existing charge layer than the magnitude of the current would be sufficient to limit the field strengths on time scales of seconds. Interestingly, the overall current measured flowing over a thunderstorm cell ranges from 0.1-6 A with a mean of approximately 0.5-i.0 A. ${ }^{32}$ While the efficiency of neutralization may be high in the positive charge layers, it is not very high in the negative regions. Furthermore, the above calculation assumes that every cosmic ray produced secondary electron finds an electric field above threshold over a surface area corresponding to a $1-\mathrm{km}$ diameter sphere and that it initiates a breakdown event that cascades over the full 300-m range of the field. As a result neutralization may occur over time scales much longer than a few seconds. On time scales of minutes or tens of minutes the field strength may well grow in the vicinity of negative charge layers and allow the development of stronger breakdown events that resemble stepped-leaders.

The cases $\delta_{0} \geq 2$ discussed above represent strong breakdown events. A comparison of the measured mean properties of stepped-leaders against the calculated parameters for the case $\delta_{0}=2$ is shown in Table $\mathrm{V}$. We find that many of the properties of individual steps in the stepped-leader process are reproducible under the appropriate conditions by the relativistic air breakdown mechanism. When the thunderstorm electric field drops below threshold, the beam deposits its charge over dimensions corresponding to the length of the beam (tens to one hundred meters) and several mean free paths (tens of meters) and on time scales corresponding to the energy loss rate (tens of nanoseconds) and the three-body attachment rate (50-400 ns, depending on the electron energy). The electric field associated with the newly formed charge layer will in general exceed the breakdown threshold, e.g., $0.38 \mathrm{C}$ of charge contained in a spherical volume of radius $75 \mathrm{~m}$ yields a field strength of $608 \mathrm{kV} / \mathrm{m}$. Thus, a high-energy electron would in the presence of this field initiate another avalanche. Assuming that the electron energy loss rate is faster than the charge deposition (electric field formation) rate, then the next avalanche will be initiated on a time scale corresponding to the local production of a high-energy secondary electron by cosmic rays. The flux of secondary electrons with energy above $1 \mathrm{MeV}$ at $5-\mathrm{km}$ altitude ranges from $200-700$ electrons $/ \mathrm{m}^{2} / \mathrm{sr} / \mathrm{s} .{ }^{33}$ A high-energy electron therefore crosses a 10-m diameter surface every 50-14 $\mu \mathrm{s}$. This analysis suggests that a stepping process would occur in which a beam first forms near the negative charge layer of the cloud, propagates several hundred meters from this layer and deposits its charge over a volume with average dimensions of one hundred meters in a time of one to two microseconds followed by the formation of a second beam after a 14-50 $\mu$ s pause, with the process repeating itself and having the net effect of moving charge towards the ground. The path to the ground would be dictated by the configuration of the electric field which in turn depends on the charge distribution defined by beam deposition plus the thunderstorm electric field. A sketch of this process is shown in Fig. 20. In general it is possible for thousands of small breakdown events to occur along a channel in the manner described above, depending on the self-consistent field magnitude and range, and in this way produce a field configuration that then allows a strong stepped-leader process to propagate from 
the cloud to the ground. While somewhat qualitative this picture of the stepped-leader process is in general agreement with optical and rf measurements. Clearly, a more quantitative model that includes a more realistic thunderstorm electric field, that incorporates the spatial transport of the electron beam and the details of charge deposition, and that includes a statistical treatment of the cosmic ray produced secondary electron flux all over a two-dimensional region between the cloud and ground is needed in order to better address the observational constraints.

TABLE V. Stepped-leader phenomenology vs calculations.

\begin{tabular}{|c|c|c|}
\hline Case & Measurements & $\delta_{0}=2.0$ \\
\hline Length $(\mathrm{m})$ & $10-200$ & 150 \\
Diameter $(\mathrm{m})$ & $1-10$ & 64 \\
Pause Interval $(\mu \mathrm{s})$ & $5-20$ & 15 \\
Current $(\mathrm{A})$ & $100-50(0)$ & 25000 \\
Line Charge Density & $10^{-3}$ & $2.5 \times 10^{-3}$ \\
$\left(\mathrm{C} \mathrm{m}^{-1}\right)$ & & \\
Rise Time $(\mu \mathrm{s})$ & $<0.1$ & 0.027 \\
\hline
\end{tabular}

Assuming a geometrical factor of $40 \mathrm{~cm}^{2} \mathrm{sr}$, the intensity of $\mathrm{x}$-rays in a $16-\mathrm{keV}$ energy bin around $100 \mathrm{keV}^{\prime}$ at $1 \mathrm{~km}$ from our electron beam source with $\delta_{0}=2$ would be $\mathrm{I}_{100 \mathrm{keV}}$ $\approx 3.1 \times 10^{4}$ photons/event and at $2 \mathrm{~km}$ from the source $I_{100} \mathrm{keV}$ (at $2 \mathrm{~km}$ ) $\approx 10$ photons/event. For photon counting systems with sufficient time resolution $(<1 \mu \mathrm{s})$, a single strong event of this type occuring within $1 \mathrm{~km}$ of the detector would more than account for the enhanced count rates observed in thunderstorms. ${ }^{11-13}$ It is also possible, however, for 1000 much weaker events occuring within $1 \mathrm{~km}$ of the detector per second and driven by thunderstorm fields such that $\delta_{0}=1.5$ over a $1 \mathrm{~km}$ diameter region with a range of $300 \mathrm{~m}$ to account for the observed count rates. The only remaining issue in this regard is a more precise identification of the $\mathrm{x}$-ray source. Simultaneous triggered and gated, high-time resolution $(<15 \mu \mathrm{s})$ measurements of $x$-ray, optical, and $\mathrm{rf}$ emissions would provide the necessary data for association of the $\mathrm{x}$-ray source with a specific lightning mechanism.

\section{CONCLUSIONS}

The single-particle analysis adopted in Paper I was based on orbit calculations and a simplified one-particle expression for secondary electron production. A more detailed analysis based on the exact kinetic theory was needed. The kinetic results presented in this paper have 
yielded some new insights into the details of the runaway breakdown mechanism. First, it is clear that the kinetic effects associated with electron transport in velocity space around the separatrix play an essential role in defining the avalanche rate. In particular, the critical energy which marks the transition to the runaway regime and defines the avalanche rate is found to be $\varepsilon_{s}\left(-1, \delta_{0}\right)$. In addition we find that angle scattering across the separatrix can also be important. Second, the detailed shape of the distribution function yields a more quantitative picture for the extent of the runaway electron beam in both energy and angle and provides a basis for estimating the spatial dimensions of the electron beam as a function of time. The latter information was used to estimate the $x$-ray flux expected from thunderstorm discharges.

The quantitative results and qualitative discussion presented in this paper clearly suggest that the relativistic breakdown mechanism first proposed in Paper I can be one of the primary processes by which electrical discharges occur in thunderstorms. The arguments in favor of this conclusion are summarized as follows: 1) the threshold electric field for this process to develop is less than the magnitude of large scale electric fields measured in active thunderstorms, 2) measurements of $x$-ray fluxes in thunderstorms are indicative of the presence of a substantial population of high-energy electrons, 3) for typical thunderstorm fields, a high-energy electron beam that propagates over hundreds of meters to kilometers is produced and accompanied by large scale breakdown of the air, 4) a substantial amount of charge is transported, 5) sufficient charge is deposited to permit formation of a new beam and to allow development of a channel to the ground, 6) many observational constraints deduced from field change meters, rf, and optical measurements of stepped-leaders are satisfied such as spatial and temporal scales, currents, and charge deposited per unit volume, and 7) the energetics of thousands of discharges (consistent with $\mathrm{rf}$ interferometric measurements ${ }^{34-36}$ ) of this type fit well within the constraints of the overall electrical energy stored in thunderstorms.

While we have provided strong quantitative and qualitative arguments supporting the notion that relativistic air breakdown plays an essential role in thunderstorm electrical discharges, it is clear that the theoretical development of our model lags substantially behind the existing observational data base. A more detailed analysis of the spatial beam transport and charge deposition for realistic thunderstorm electric field configurations in at least two dimensions is necessary in order to develop a more realistic model. Calculations of the expected optical and rf emissions from such a model would then permit a more detailed comparison with existing data. It would also be useful to re-examine existing observations in the light of this new theory and to field new diagnostics to look for the presence of high-energy electrons in the stepped-leader process. Measurements of the x-ray flux within 1 to $2 \mathrm{~km}$ of a developing stepped-leader to corroborate the measurements of Shaw 8 would be useful, for example. Flights of $x$-ray spectrometers with $\mu$ s time resolution through thunderclouds would help to resolve individual discharges. Sub-microsecond photometry of lightning discharges in specific nitrogen lines such as $\mathrm{N}_{2} \lambda 3371$ and $\mathrm{N}_{2}+\lambda 3914$ when compared with measured spectra of electron beam 
fluorescence in air ${ }^{37-38}$ may provide additional evidence for the existence of high-energy electrons. One essential ingredient in any model that attempts to reproduce lightning discharges is an accurate specification of the thunderstorm electric field configuration. Development of reliable remote sensing techniques to map out the field strength over large scales (kilometers) is extremely important. Radar mapping of polarized ice crystals in thunderstorms may be useful for this purpose. ${ }^{39}$

Finally, we hope that the results presented in this paper and Paper I will provide new incentive for further examination of lightning discharges as a whole occurring both on earth and in other planetary systems and that corroborating laboratory measurements of this new breakdown mechanism will be performed in the near future.

\section{ACKNOWLEDGMENTS}

The authors would like to thank Dr. C. Rhodes for helpful discussions regarding lightning phenomenology and Dr. G. Shaw for providing a historical perspective on the measurements of runaway electrons. 


\section{REFERENCES}

${ }^{1}$ A.V. Gurevich, G.M. Milikh, R.A. Roussel-Dupré, Phys. Lett. A 165, 463 (1992).

${ }^{2}$ C.T.R. Wilson, Proc. Cambridge Philos. Soc. 22, 534 (1924).

${ }^{3}$ C.T.R. Wilson, Proc. Phys. Soc. London 37, 320 (1925).

${ }^{4}$ B.F.J. Schonland, Proc. R. Soc. London A 130, 37 (1930).

${ }^{5}$ B.F.J. Schonland and J.P.T. Viljoen, Proc. R. Soc. London A 140, 314 (1933).

6E.C. Halliday, Proc. Cambridge Philos. Soc. 30, 206 (1934).

7W.A. Macky, Proc. Cambridge Philos. Soc. 30, 70 (1934).

8R.D. Hill, J. Geophys. Res. 68, No. 23, 6261 (1963).

${ }^{9}$ G.E. Shaw, J. Geophys. Res. 72, No. 18, 4623 (1967).

10D.P. Whitmire, Lett. Nuovo Cimento 26, 497 (1979).

${ }_{11}$ G.K. Parks, B.H. Mauk, R. Spiger, and J. Chin, Geophys. Res. Lett. 8, No. 11, 1176 (1981).

12M.P. McCarthy and G.K. Parks, Geophys. Res. Lett. 12, No. 6, 393 (1985).

13M.P. McCarthy and G.K. Parks, J. Geophys. Res. 97, No. D5, 5857 (1992).

${ }^{14}$ N. D'Angelo, Ann. Geophysicae 5B, (2), 119 (1987).

${ }^{15}$ H. A. Bethe, Ann. Phys. 5, 325 (1930).

${ }^{16}$ H.A. Bethe and J. Ashkin, in Experimental Nuclear Physics, edited by E. Segre, 1, (John Wiley and Sons, New York, 1953), p. 277.

${ }^{17}$ T. Murphy, Los Alamos National Laboratory Report no. LA-11288-MS (1988).

${ }^{18}$ C. B. Opal, W.K. Peterson, and E.C. Beaty, J. Chem. Phys. 55, 4100 (1971).

${ }^{19}$ C. B. Opal, E.C. Beaty, and W.K. Peterson, At. Data 4, 210 (1972).

${ }^{20} \mathrm{C}$. Moller, Ann. Phys. (Leipzig), 14, 531 (1932).

${ }^{21}$ S. Chapman and T.G. Cowling, The Mathematical Theory of Non-Uniform Gases, (Cambridge University Press, London, 1970), p. 400.

${ }^{22}$ C.L. Longmire and H.J. Longley, Defense Nuclear Agency report no. DNA 3192T (1973).

${ }^{23}$ B.M. Isaev, I.V. Gordeev, Yu. I Bregadze, Yu.S. Gerasimov, and G.N. Antipenhova, Izmeritel'naya Teknika English Trans., 389 (1967).

${ }^{24}$ H.A. Bethe and J. Ashkin, in Experimental Nuclear Physics, edited by E. Segrè, 1, (John Wiley and Sons, New York, 1953), p. 233.

${ }^{25}$ H.A. Bethe and W. Heitler, Proc. R. Soc. London A 146, 83 (1934).

26W. Heitler, The Quantum Theory of Radiation, (Clarendon Press, Oxford, 1954), p. 245.

27J.D. Jackson, Classical Electrodynamics, Second Edition (John Wiley and Sons, New York, 1975), p. 713.

${ }^{28}$ In American Institute of Physics Handbook, edited by D.E. Gray, Third Edition (McGraw Hill, New York, 1972), p. 8-203.

${ }^{29}$ W.P. Winn, C.B. Moore, and C.R. Holmes, J. Geophys. Res. 86, No. C2, 1187 (1981).

30W.P. Winn, G.W. Schwede, and C.B. Moore, J. Geophys. Res. 79, No. 12, 1761 (1974). 
31P.R. Krehbiel, in Th: Earth's Electrical Environment, (National Academy Press, Washington DC, 1986), p. 90.

32R.R. Roble and Israel Tzur, in The Earth's Electrical Environment, (National Academy Press, Washington DC, 1986), p. 206.

${ }^{33}$ R. R. Daniel and S.A. Stephens, Rev, of Geophys. and Spa. Phys. 12, No, 2, 233 (1974).

${ }^{34}$ C.O. Hayenga and J.W. Warwick, J. Geophys. Res. 86, No. C8, 7451 (1981).

${ }^{35}$ D.E. Proctor, J. Geophys. Res. 88, No. C9, 5421 (1983).

36C.T. Rhodes and P.R. Krehbiel, Geophys. Res. Lett. 16, 1169 (1989).

${ }^{37}$ G. Davidson and R. O'Neil, J. Chem. Phys. 41, 3946 (1964).

${ }^{38}$ R.R. Karl, Jr., Appl. Opt. 28, No. 3, 446 (1989).

${ }^{39}$ A. Hendry and G.C. McCormick, J. Geophys. Res. 81, No. 30, 5353 (1976). 


\section{FIGURE CAPTIONS}

FIG. 1. Configuration space geometry. The applied electric field is assumed to be in the $\mathrm{z}$-direction. An electron with momentum $\mathrm{P}$ is directed at an angle $\theta$ to the field.

FIG. 2. Scaled dynamical friction. The magnitude of the dynamical friction force is plotted as a function of electron energy.

FIG. 3. Ionizing collision geometry. In Figure 3(a) an incident electron of energy $\varepsilon$ ' collides with an air molecule and scatters with energy $\varepsilon_{s}$. A secondary electron of energy $\varepsilon$ is produced at an angle $\xi$ relative to the incident electron. The azimuthal angle $\alpha$ is measured in the plane perpendicular to that containing the incident and scattered electrons. Conservation of energy requires that $\varepsilon_{s}=\varepsilon^{\prime}-\varepsilon-\varepsilon$, where $\varepsilon_{t}$ is the ionization energy. The equivalent collision geometry in the lab frame is depicted in Figure 3(b). The Z-axis corresponds to the direction of the applied electric field.

FIG. 4. Total ionization cross-section. The total ionization cross-section is plotted as a function of electron energy.

FIG. 5. Relativistic Separatrix for $\delta_{0}=2$. The minimum initial electron energy needed for runaway for $\delta_{0}=2$ is plotted as a function of the initial electron direction of motion relative to the applied electric field. The parameter $\mu_{s}$ is defined to be the cosine of the angle between the initial momentum of the electron and the electric field.

FIG. 6. Minimum energy for runaway. The minimum runaway energy necessary for an electron whose initial momentum is perpendicular [Fig. 6(a)] or antiparallel [Fig. 6(b)] to the electric field is plotted as a function of $\delta_{0}$.

FIG.7. Particle trajectory in velocity space. The particle kinetic energy normalized to $\mathrm{mc}^{2}$ is plotted as a function of $\mu$ ( $=$ cosine of the angle between the particle momentum and the electric field) for an initial electron kinetic energy of $50 \mathrm{keV}$ [Fig. 7(a)] and 50) keV [Fig. 7(b)] and for five initial $\mu$-values. The electric field strength was taken to be $\delta_{0}=2$.

FIG. 8. Particle orbits in velocity space vs time. The particle kinetic energy normalized to mc ${ }^{2}$ is plotted as a function of time (in ns) for an initial electron kinetic energy of $50 \mathrm{keV}$ [Fig. 8(a)] and $500 \mathrm{keV}$ [Fig. $8(\mathrm{~b})$ ] and for five initial $\mu$-values. The electric tield strength was taken to be $\delta_{0}=2$. 
FIG. 9. Particle orbits in velocity space vs time. The cosine of the angle between the particle momentum and the electric field is plotted as a function of time (in ns) for an initial electron kinetic energy of $50 \mathrm{keV}$ [Fig. 9(a)] and $500 \mathrm{keV} \mathrm{[Fig.} \mathrm{9(b)]} \mathrm{and} \mathrm{for} \mathrm{five} \mathrm{initial} \mu$-values. The electric field strength was taken to be $\delta_{0}=2$.

FIG. 10. Particle trajectory in configuration space. The electron trajectory in the presence of an electric field applied along the minus z-direction is plotted. Note that the scale in the direction perpendicular to the applied field is much smaller than that along the field. This has the effect of distorting the particle orbit so that the angle between the particle motion and the electric field looks much larger than in reality. The electric field strength was taken to be $\delta_{0}=2$.

FIG. 11. Time history of the overall electron distribution function. The amplitude of the overall electron distribution function obtained for $\delta_{0}=2$ is plotted as a function of $\log \varepsilon$ and $\mu$ for various times including (a) $t=0.0$, (b) $t=1 .($, (c) $t=1.5$, (d) $t=20$, (c) $t=5($, and $(\mathrm{f}) \mathrm{t}=15($ ) ns.

FIG. 12. Time history of the beam distribution function. The amplitude of the beam distribution function obtained for $\delta_{0}=2$ is plotted as a function of $\log \varepsilon$ and $\mu$ for various times including (a) $t=0.0$, (b) $t=1.0$, (c) $t=1.5$, (d) $t=20$, (e) $t=50$, and (f) $t=150$ ns.

FIG. 13. Time history of the heam distribution function. The amplitude of the beam distribution function obtained for $\delta_{0}=2$ is plotted as a function of log $\varepsilon$ for five angles measured relative to the electric field and for various times including (a) $t=0 .()$, (b) $t=1.0$, (c) $t=1.5$, (d) $t=2($ ), (e) $\mathrm{t}=50$, and $(\mathrm{f}) \mathrm{t}=150 \mathrm{~ns}$.

FIG. 14. Moments of the overall and beam distribution functions. The overall electron density, mean kinetic energy, and kinetic energy spread are plotted as a function of time in (a)-(c), respectively. The beam electron density, mean kinetic energy, and kinetic energy spread are plotted as a function of time in (d)-(f), respectively.

FIG. 15. Self-similar beam distribution function. The self-similar beam distribution function is plotted as a function of electron energy for five ungles measured relative to the applied electric field and for three values of the field including, (a) $\delta_{0}=2.0$, (b) $\delta_{0}=5.0$, and (c) $\delta_{0}=8 .($ ).

FIG. 16. Ratio of computed ionization rates to analytic estimates of rates. The ratio of the computed ionization rate for the overall population of electrons (solid curve) and separately for the runaway electrons (dashed curve) to the corresponding analytic estimate is plotted as a function of time for three values of the applied field, (a) $\delta_{0}=2 .(),(b) \delta_{0}=5 .()$, (c) $\delta_{0}=8 .($ ). 
FIG. 17. Spatial dimension of electron beam. A cartoon of the beam evolution along the applied electric field is shown in (a). The length of the beam after a time $t$ is given as the dispersion in beam velocity $\Delta v$ multiplied by the time, $L=\Delta v \mathrm{l}$. The evolution of the beam perpendicular to the applied field is shown in (b). The beam diameter is depicted as a random walk in which secondary electrons ejected perpendicular to the beam direction are accelerated back into the direction of the beam within a perpendicular distance $L_{\text {step. }}$. The number of steps taken in a time $t$ is defined as $v_{t i}$ where $t_{i}$ is an ionization time.

FIG. 18. Beam profile after 40 e-foldings. The beam density profile is plotted as a function of the number of step lengths from the position of the initial seed electron. The average step-length is taken to be the lateral distance travelled hy an electron in an ionization time including the effects of angular scattering and perpendicular ejection of secondary electrons. These results were obtained by numerically simulating the random walk depicted in Fig. 17(b), starting with a single particle and extending the calculation to $4(0)$-foldings.

FIG. 19. Bremsstrahlung emission. The specific intensity calculated for $\delta_{0}=2$ and normalized to a distance of $1 \mathrm{~km}$ is plotted in (a) as a function of the log of the photon energy and the emission angle measured relative to the applied field. The specific intensity calculated for $\delta_{0}=2$ and normalized to a distance of $1 \mathrm{~km}$ is plotted in (b) as a function of the emission angle for five photon energies. Atmospheric attenuation is not included in these calculations.

FIG. 20. Sketch of a potential model for the stepped-leader process. The basic geometry envisioned for the electrical structure of a cumulonimbus cloud is shown on the left. Intracloud discharges are sketched along with a completed stepped-leader from cloud-to-ground. A blowup of a small section (corresponding to tens to hundreds of meters) of the leader is shown on the right. The old beam originated from above, left positive charge behind along its ionizing path, and deposited its charge in the form of negative ions. The electric field resulting from this layer of charge then accelerates a new secondary electron originating from a cosmic ray shower and produces a new electron beam that generates additional ionization along its path and deposits charge farther down stream in a direction dictated by the overall electric field patterns. This process is repeated many times, proceeding down to the ground where the return stroke is initiated. 


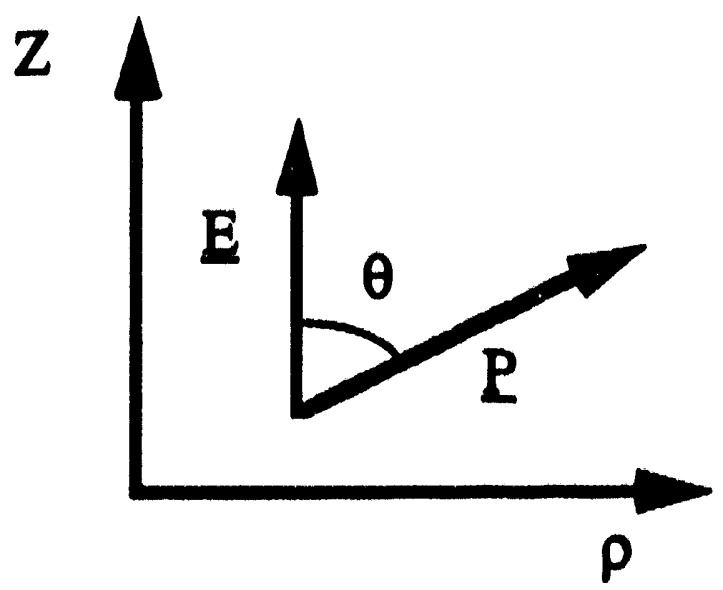

Figure 1. Configuration space geometry. 


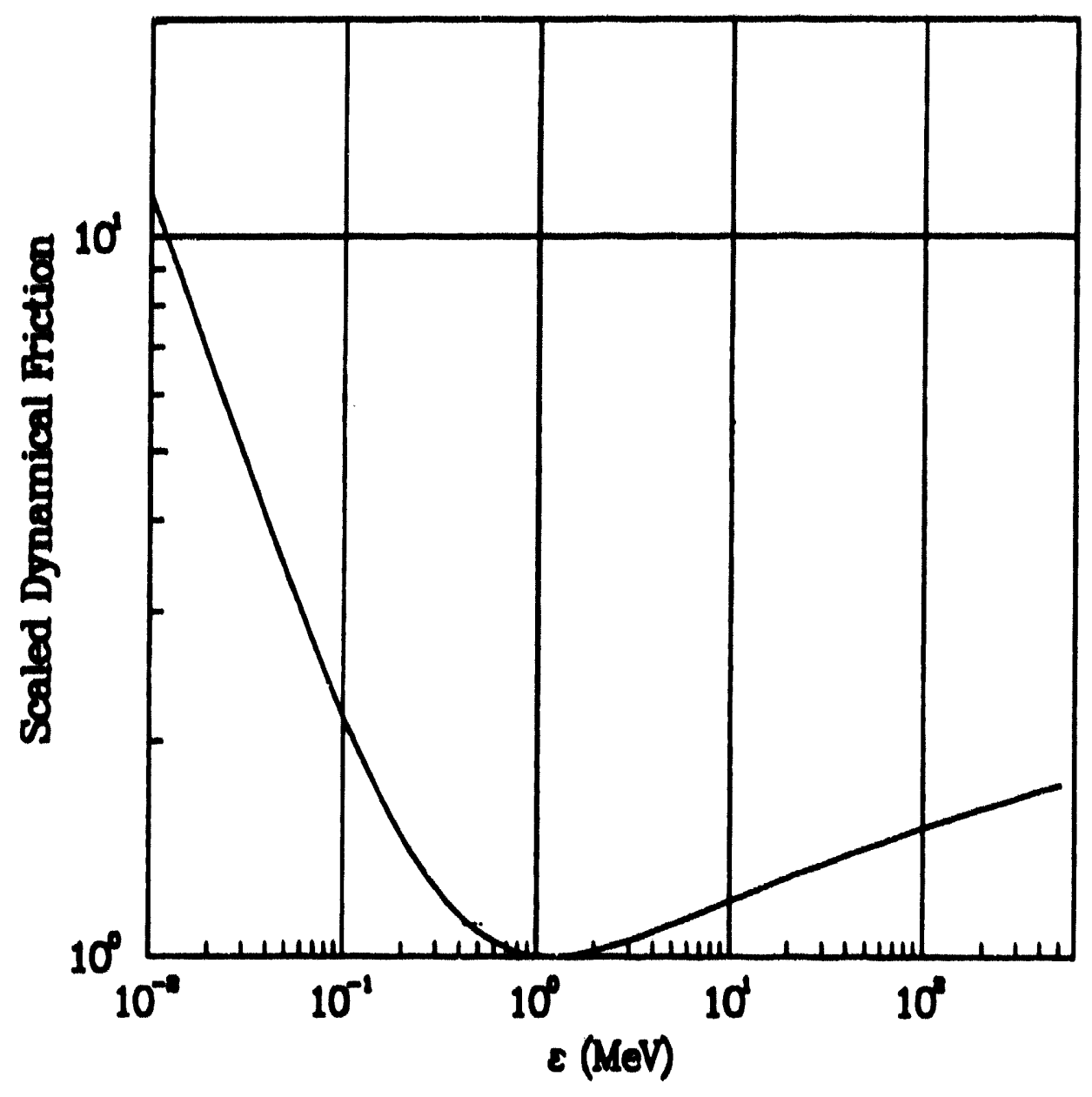

Figure 2. Scaled dynamical friction. 


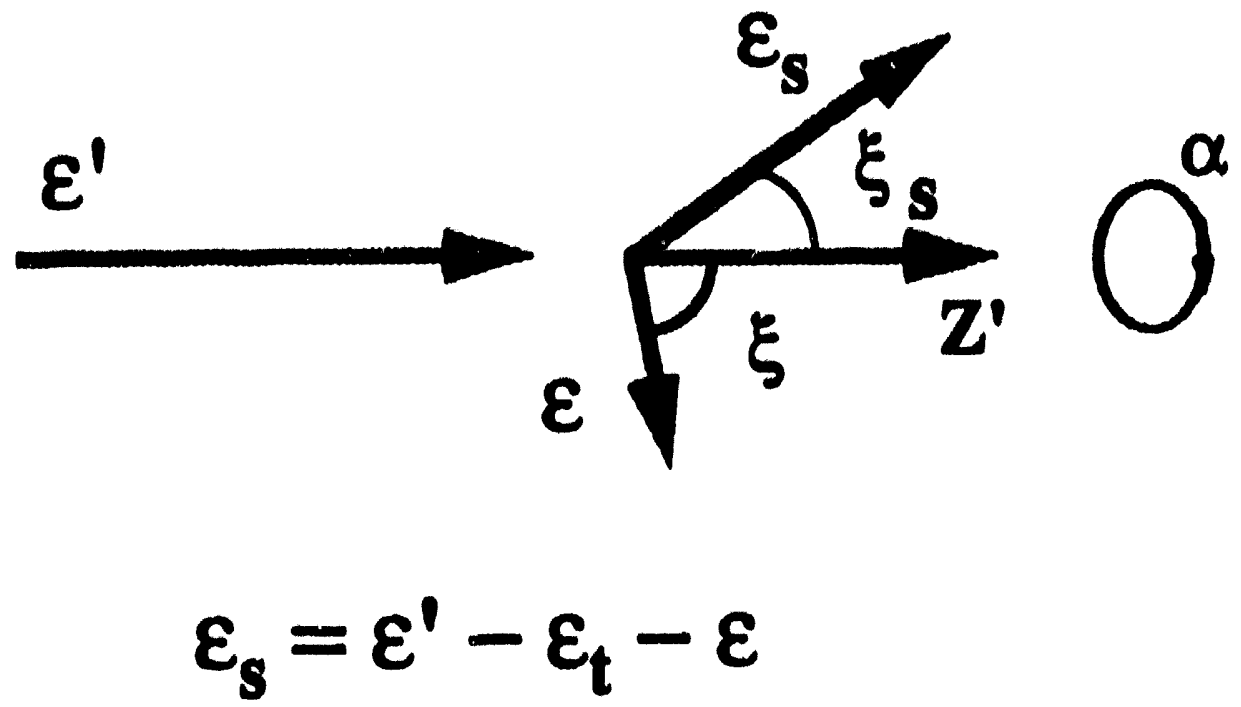

Figure 3a. lonizing collision geometry, center of mass coordinates.

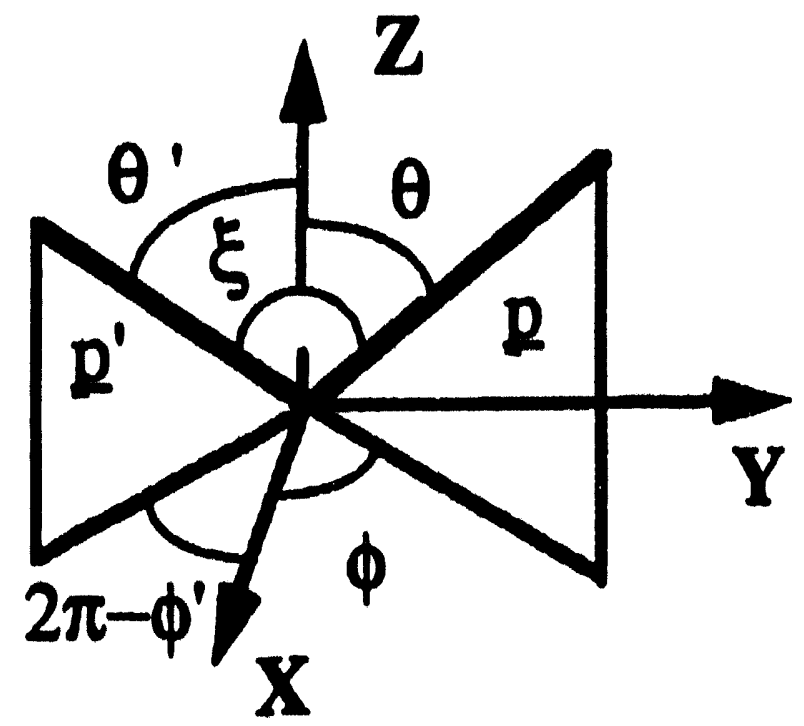

Figure 3b. Ionizing collision geometry, lab frame coordinates. 


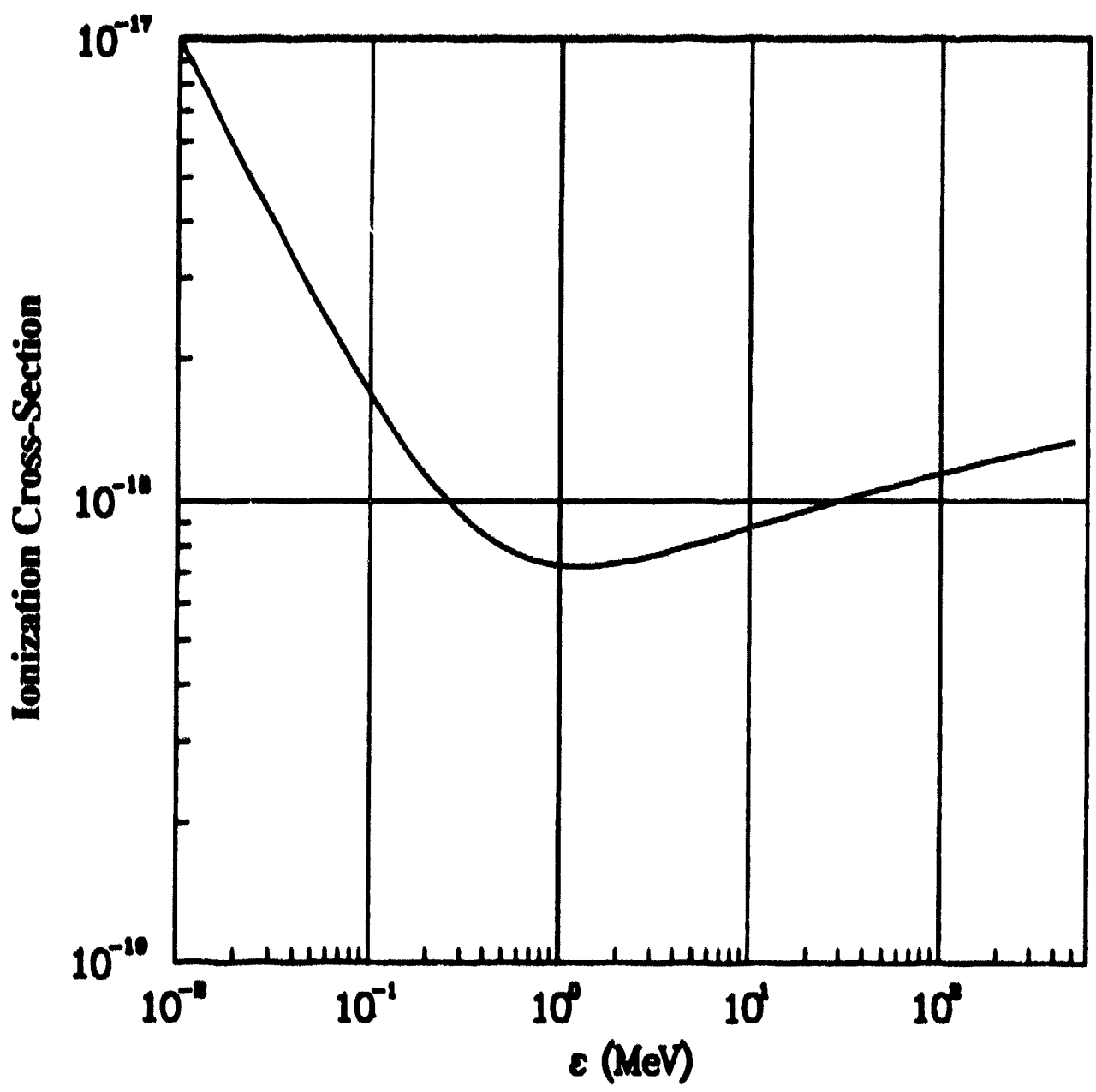

Figure 4. Ionization cross-section. 


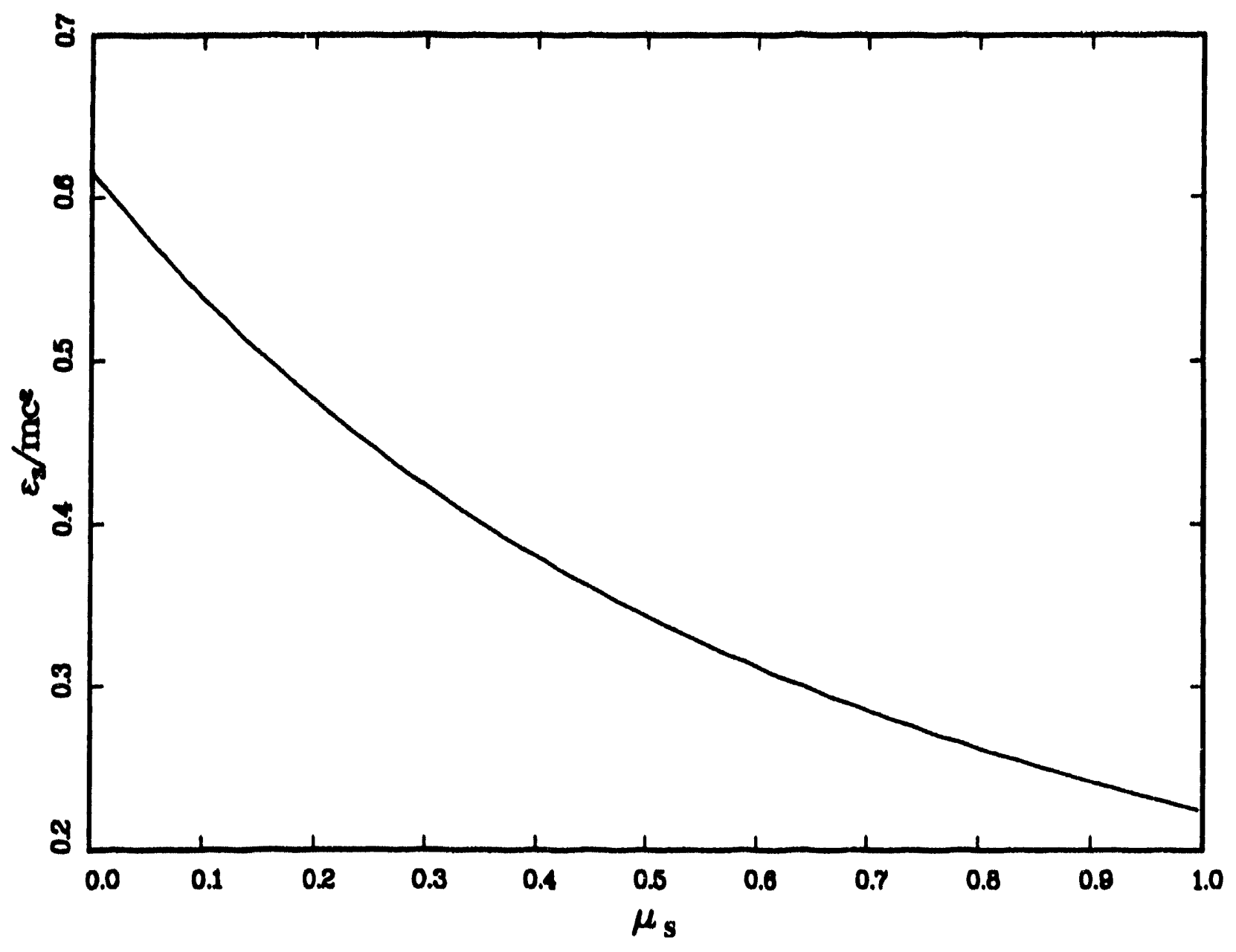

Figure 5. Relativistic Separatrix $\delta_{0}=2$. 


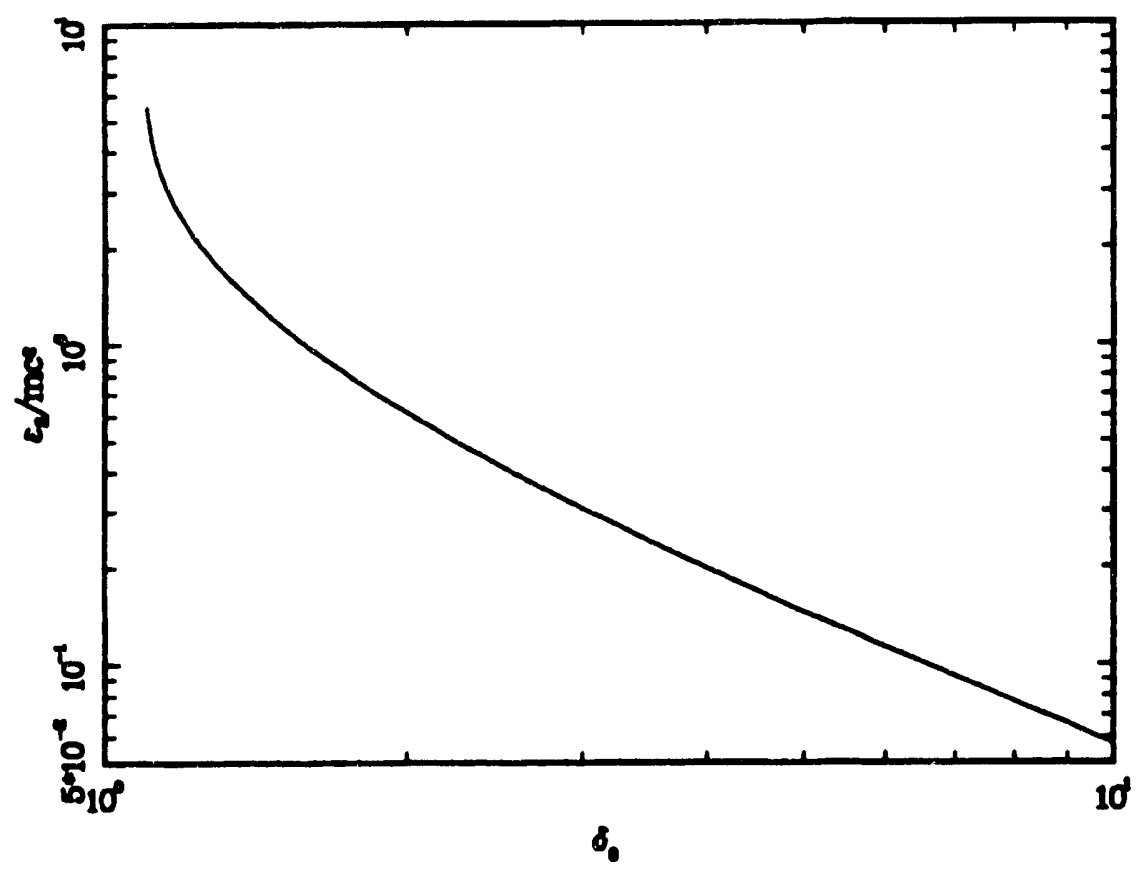

Figure 6a. Minimum energy for electron injected perpendicular to the applied field to runaway.

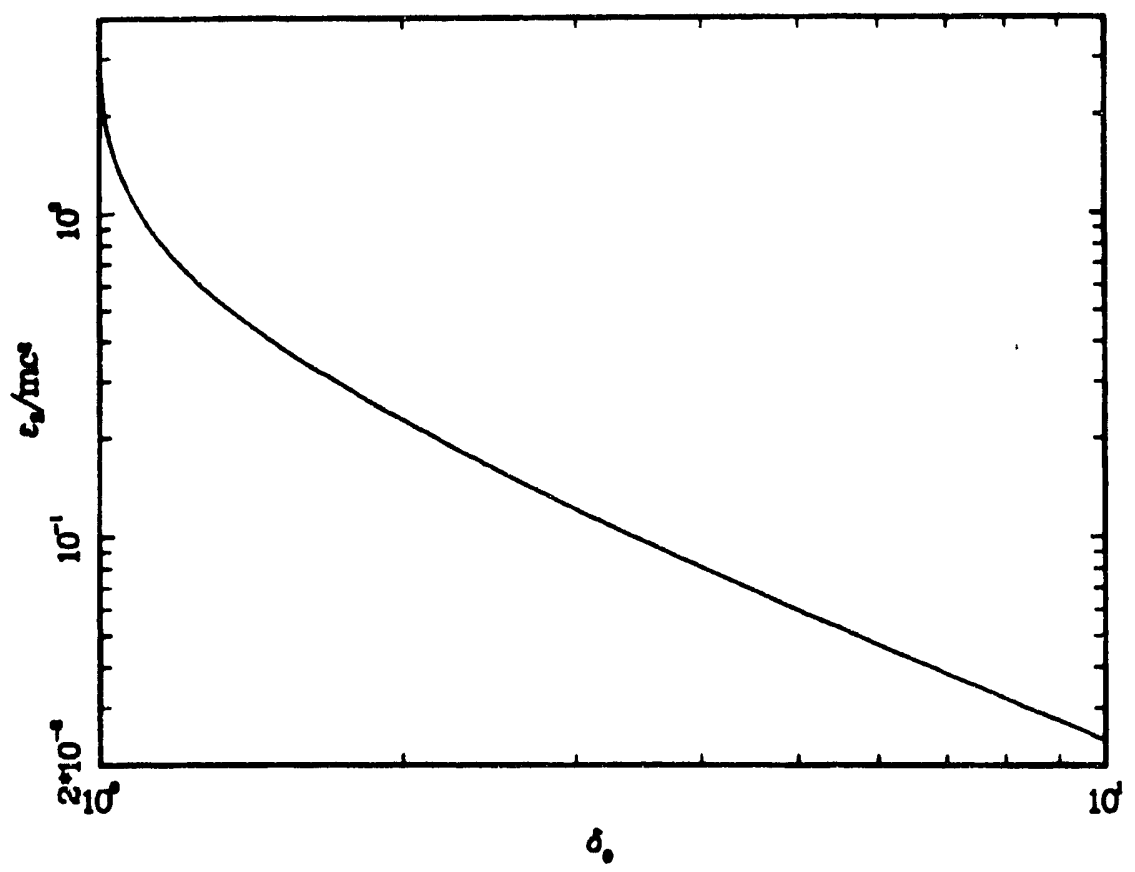

Figure 6b. Minimum energy for electron injected antiparallel to the applied field to runaway.

Figure 6. Minimum energy for "runaway". 


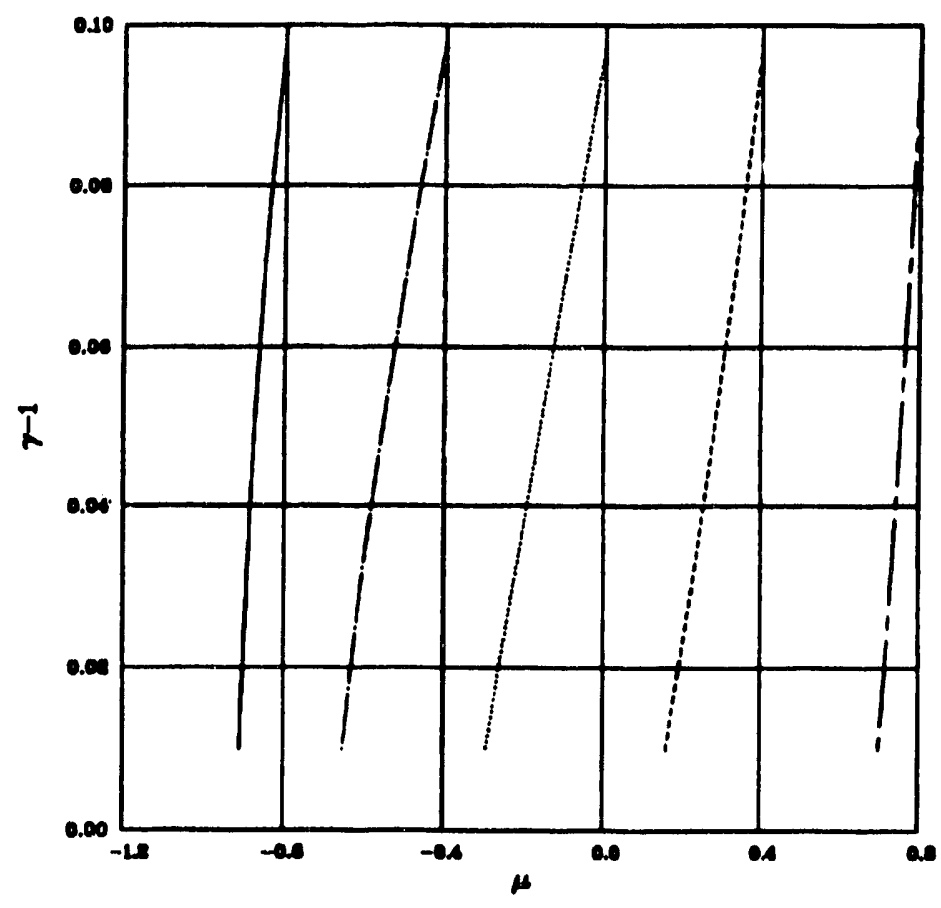

Solld $\quad: \mu_{1}=-0.80$

Chain-dot : $\mu_{1}=-0.40$

Dotted $: \mu_{1}=0.00$

Danhed $: \mu_{1}=0.40$

Chain-danh : $\mu_{1}=0.80$

Figure 7a. $\varepsilon_{\mathrm{i}}=50 \mathrm{keV}$.

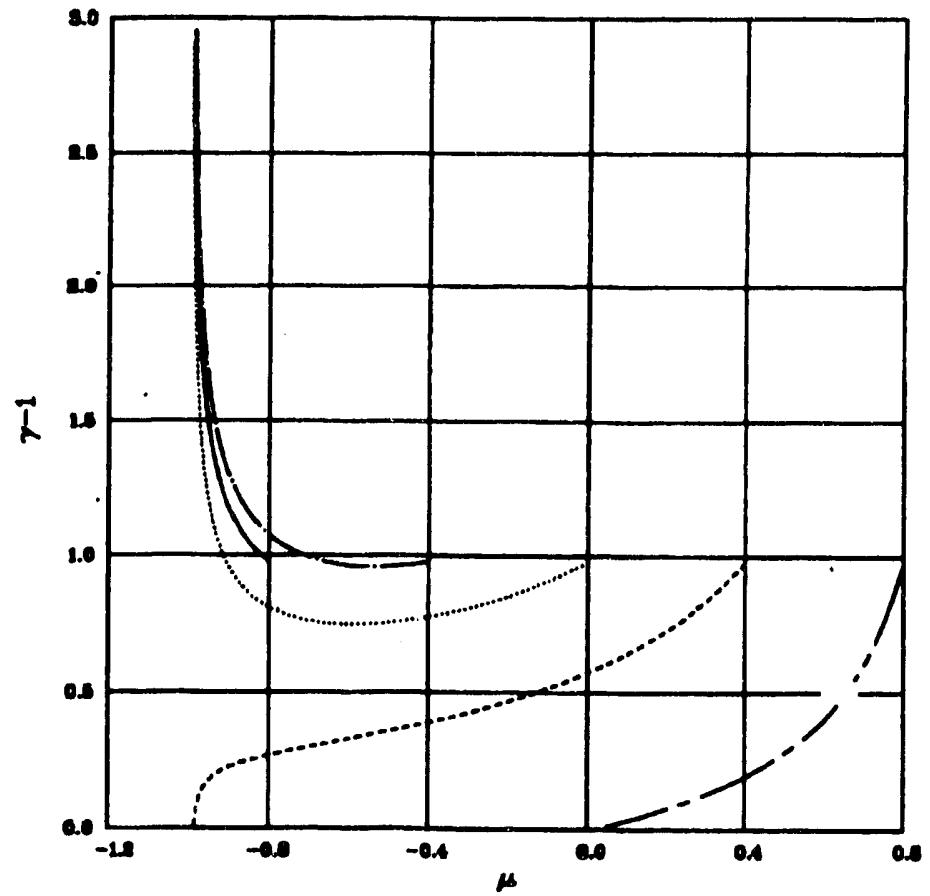

$$
\begin{array}{ll}
\text { Solid } & : \mu_{1}=-0.80 \\
\text { Chain-dot } & : \mu_{1}=-0.40 \\
\text { Dotted } & : \mu_{1}=0.00 \\
\text { Danbed } & : \mu_{1}=0.40 \\
\text { Chaln-dash }: \mu_{1}=0.80
\end{array}
$$

Figure $7 \mathrm{~b} . \varepsilon_{\mathrm{i}}=500 \mathrm{keV}$.

Figure 7. Particle trajectory in velocity space. 


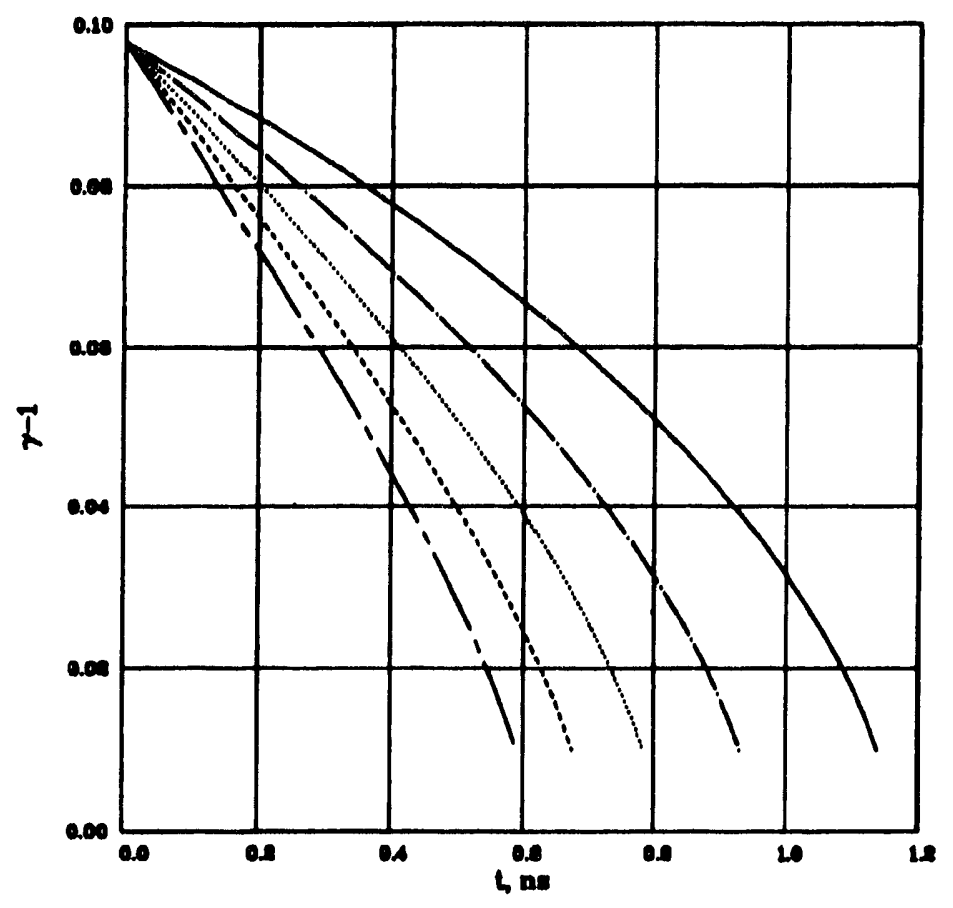

$\begin{array}{ll}\text { Solld } & : \mu_{1}=-0.80 \\ \text { Chain-dot } & : \mu_{1}=-0.40 \\ \text { Dotted } & : \mu_{1}=0.00 \\ \text { Daghed } & : \mu_{1}=0.40 \\ \text { Chain-danh } & : \mu_{1}=0.80\end{array}$

Figure $8 \mathrm{a} . \varepsilon_{\mathrm{i}}=50 \mathrm{keV}$.
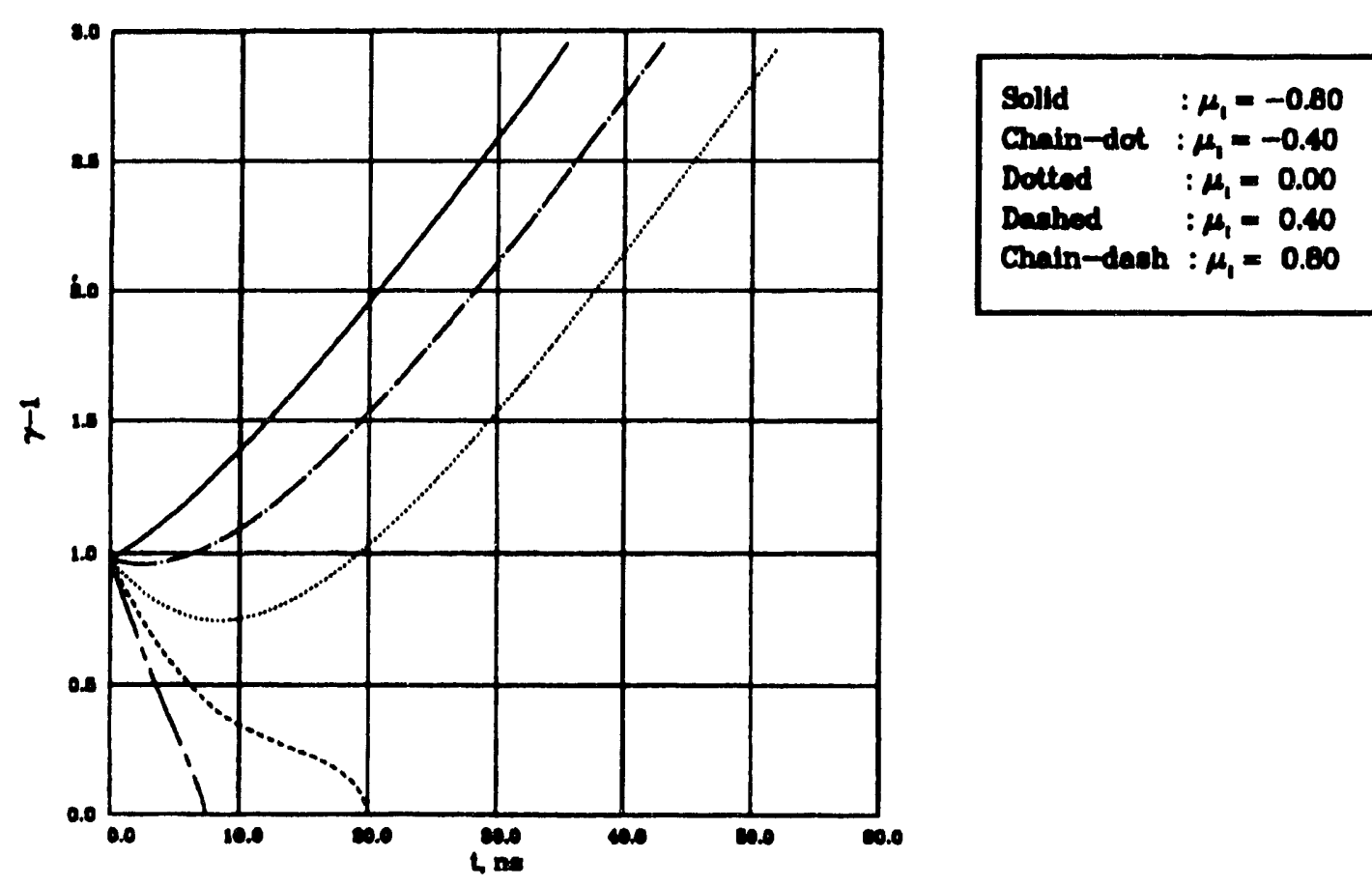

Figure $8 b . \varepsilon_{i}=500 \mathrm{keV}$.

Figure 8. Particle orbits in velocity space vs time. 


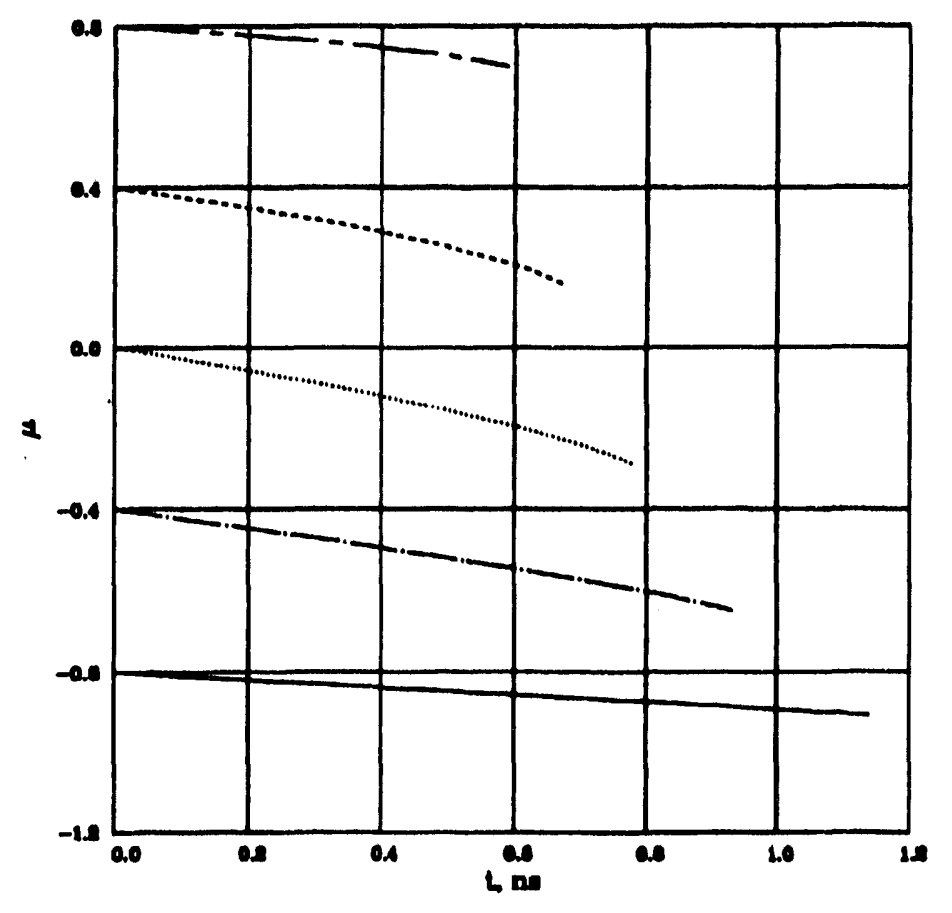

$\begin{array}{ll}\text { Solld } & : \mu_{1}=-0.80 \\ \text { Chaln-dot } & : \mu_{1}=-0.40 \\ \text { Dotted } & : \mu_{1}=0.00 \\ \text { Danbed } & : \mu_{1}=0.40 \\ \text { Chnin-danh }: \mu_{1}=0.80\end{array}$

Figure 9a. $\varepsilon_{\mathrm{i}}=50 \mathrm{keV}$.

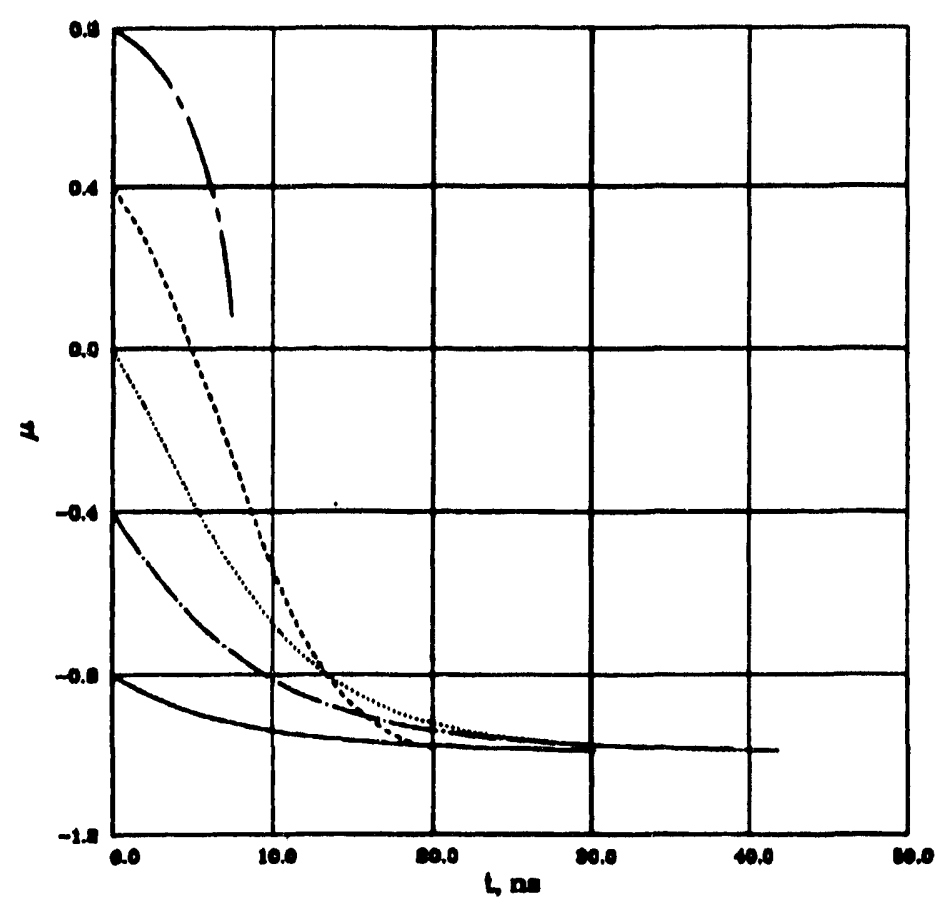

$\begin{array}{ll}\text { Solyd } & : \mu_{1}=-0.80 \\ \text { Chaln-dot } & : \mu_{1}=-0.40 \\ \text { Dotted } & : \mu_{1}=0.00 \\ \text { Danhad } & : \mu_{1}=0.40 \\ \text { Chnin-danh }: \mu_{1}=0.80\end{array}$

Figure $9 \mathrm{~b} . \varepsilon_{\mathrm{i}}=500 \mathrm{keV}$.

Figure 9. Particle orbits in velocity space vs time. 


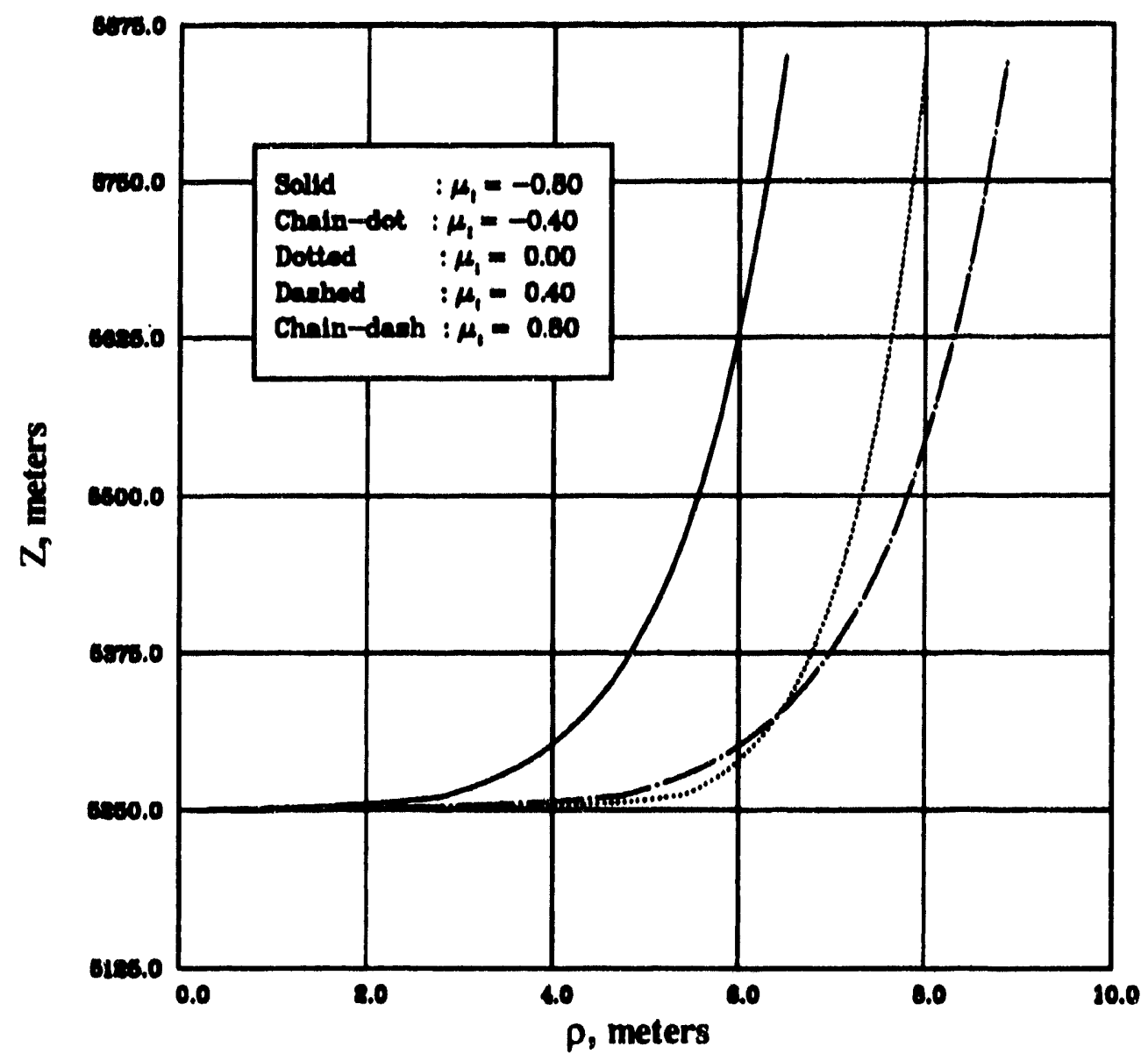

Figure 10. Particle trajectory in configuration space.

(altitude vs range tracjectory $\varepsilon_{\mathrm{i}}=500 \mathrm{keV}$ and $\delta_{0}=2$ ). 


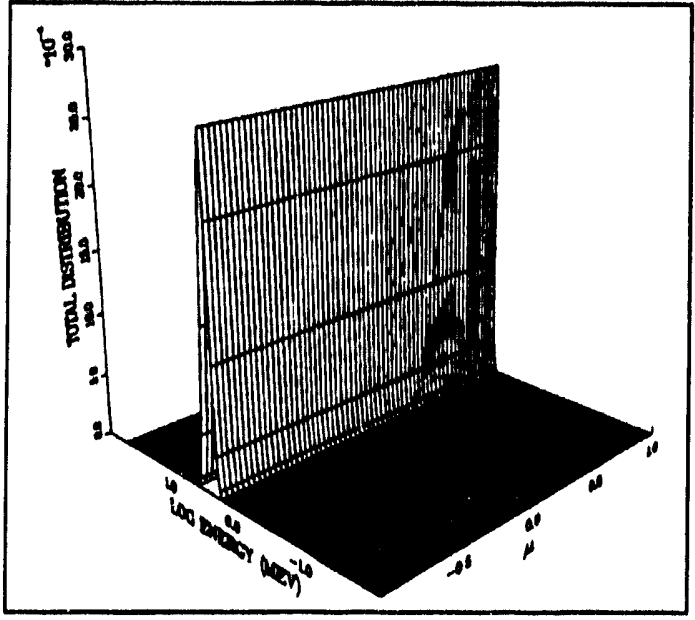

Figure 11a. $\mathrm{t}=0.00 \mathrm{~ns}$

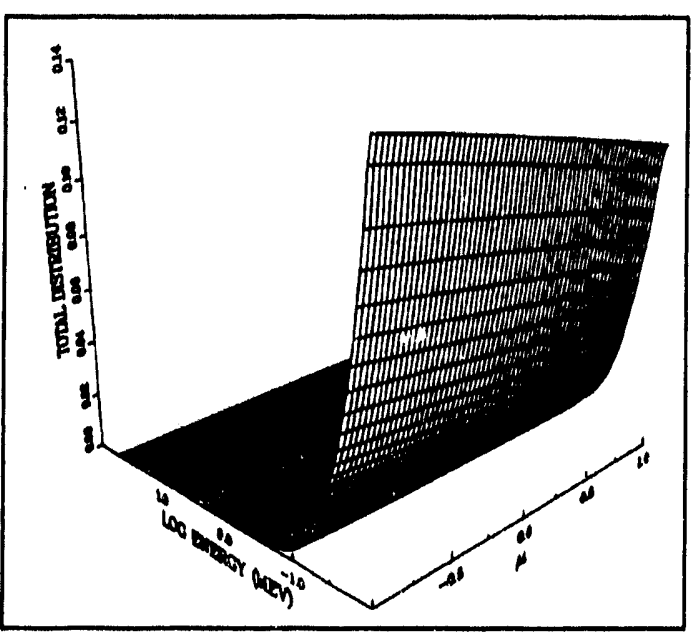

Figure 11b. $\mathrm{t}=1.00 \mathrm{~ns}$

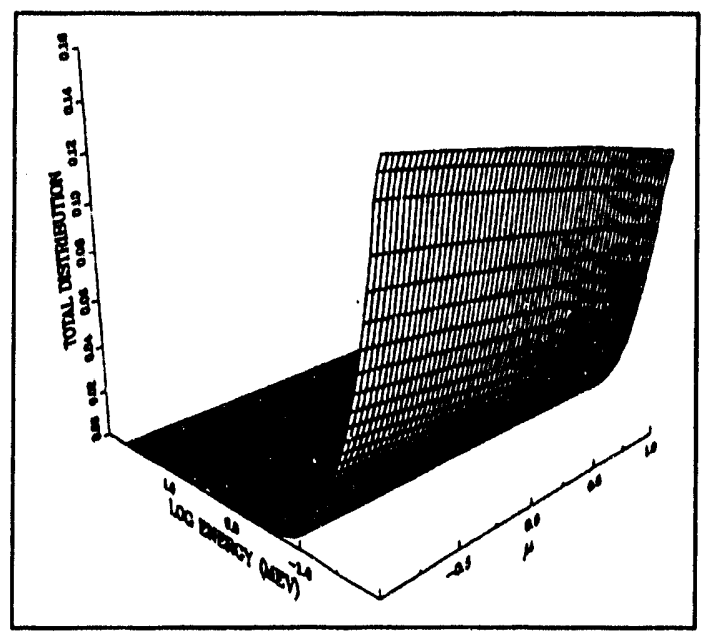

Figure 11c. $\mathrm{t}=1.50 \mathrm{~ns}$

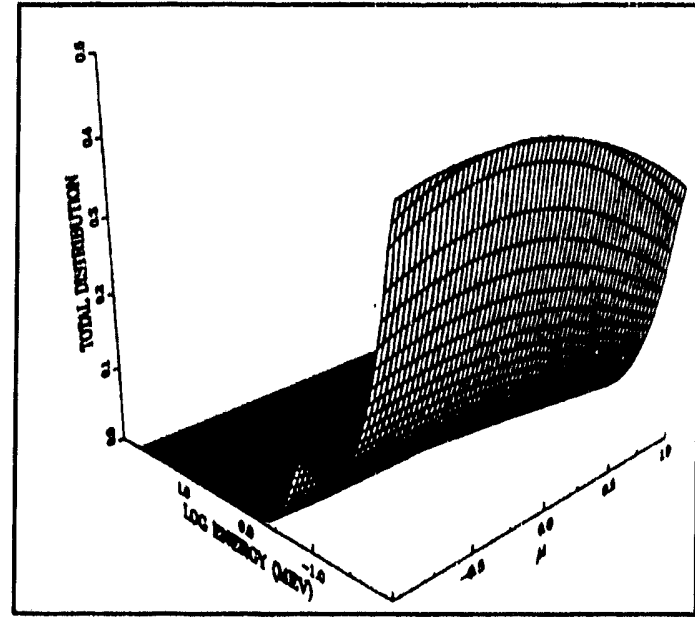

Figure 11d. $t=20.0 \mathrm{~ns}$

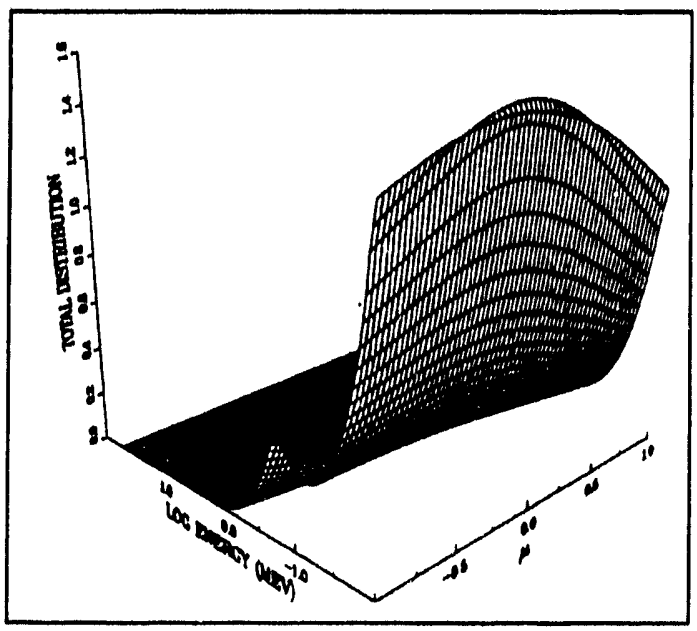

Figure 11e. $t=50.0 \mathrm{~ns}$

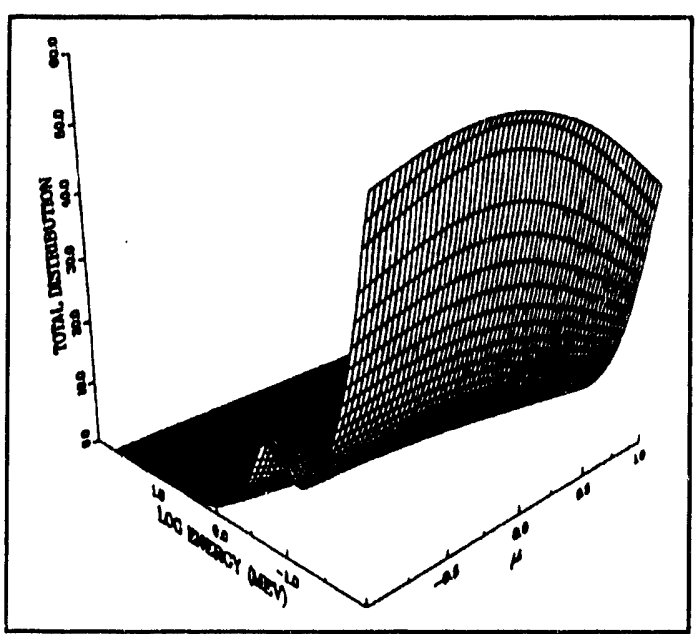

Figure 11f. $t=150.0 \mathrm{~ns}$

Figure 11. Time history of the overall electron distribution function. 


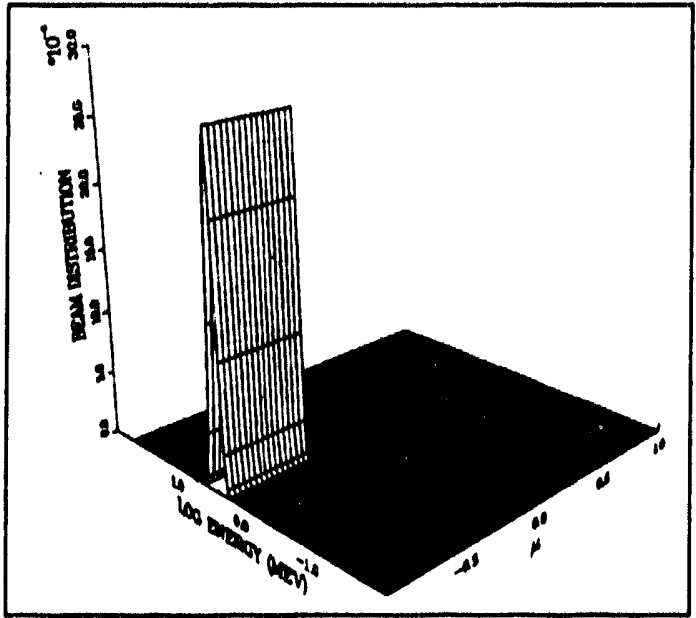

Figure 12a. $t=0.00 \mathrm{~ns}$.

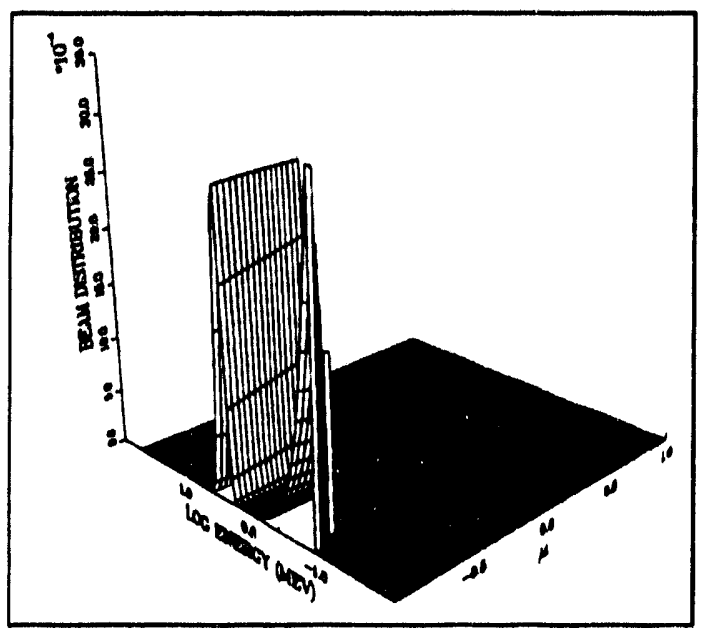

Figure 12b. $t=1.00 \mathrm{~ns}$.

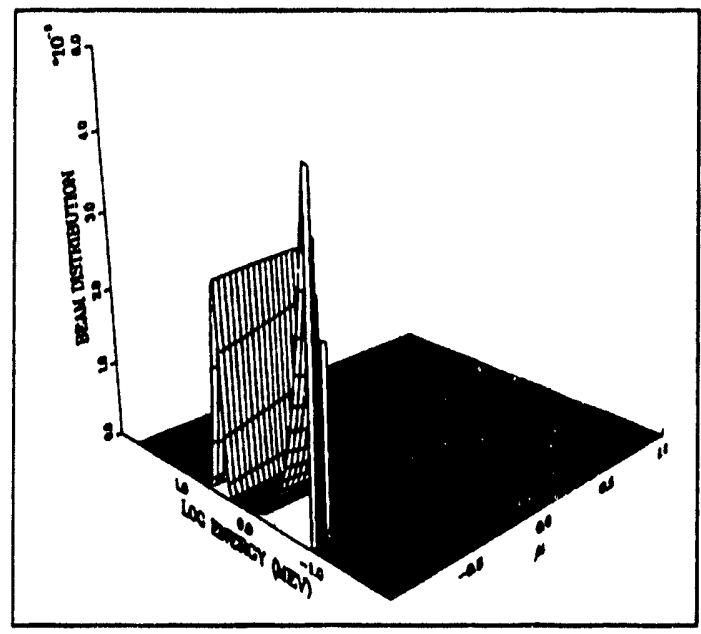

Figure 12c. $t=1.50$ ns.

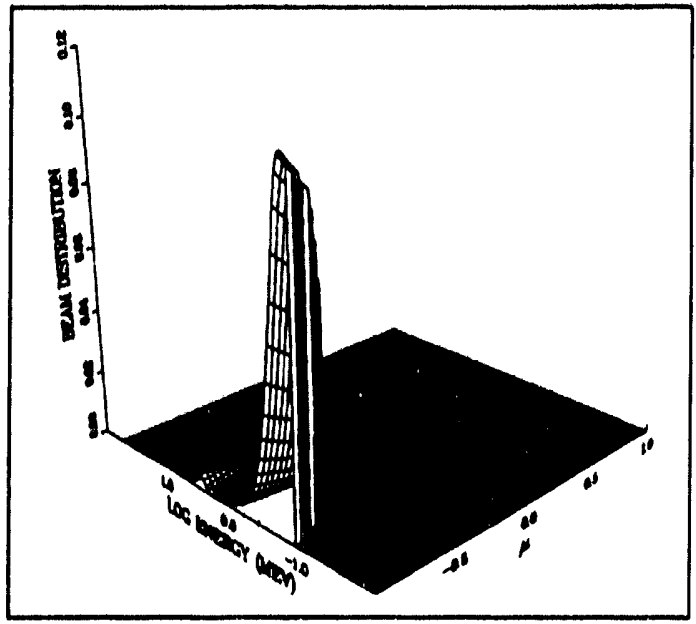

Figure $12 d . t=20.0$ ns.

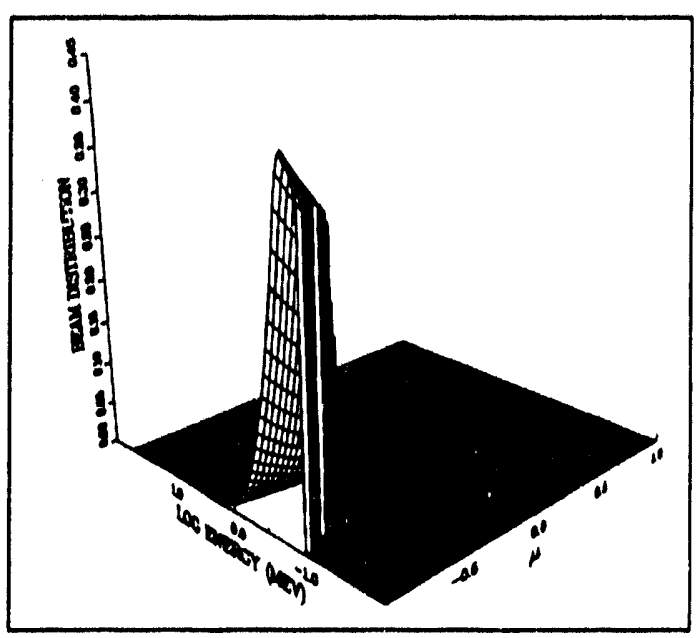

Figure 12e. $\mathrm{t}=50.0 \mathrm{~ns}$.

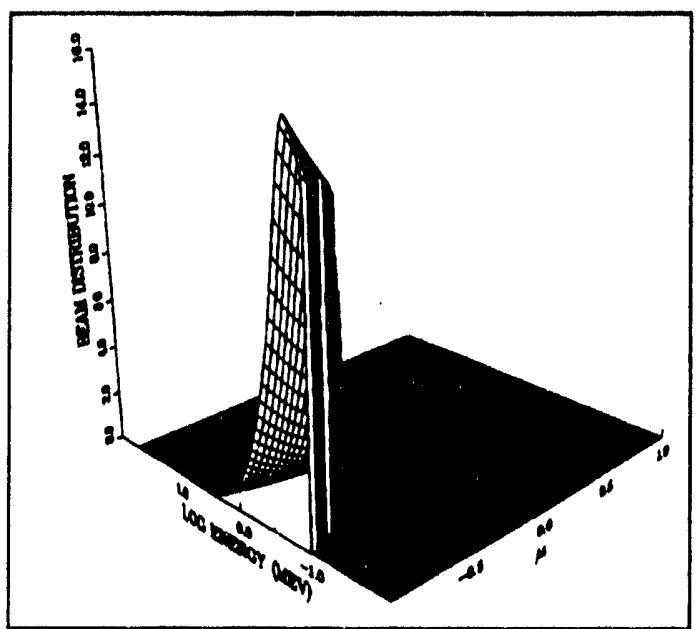

Figure 12f. $t=150.0$ ns.

Figure 12. Time history of the beam distribution function. 


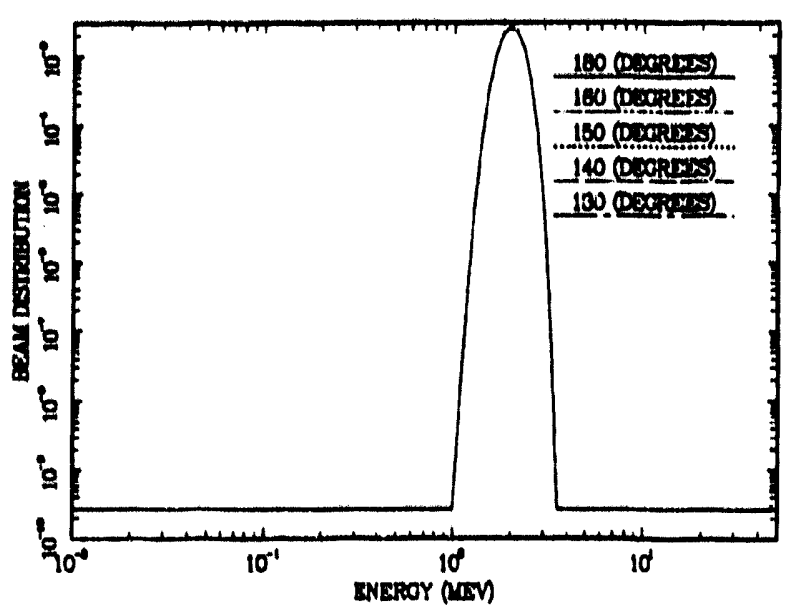

Figure 13a. $t=0.00$ ns.

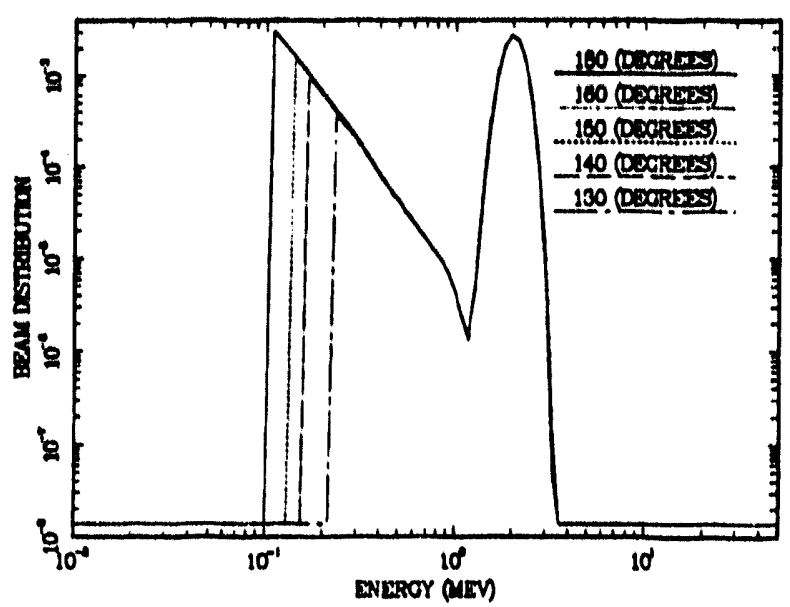

Figure 13b. $t=1.00 \mathrm{~ns}$.

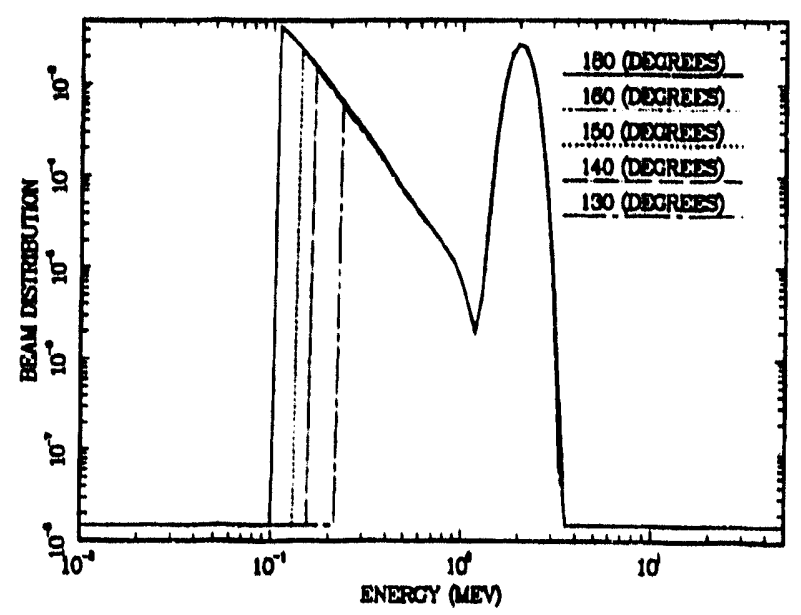

Figure 13c. $t=1.50$ ns.

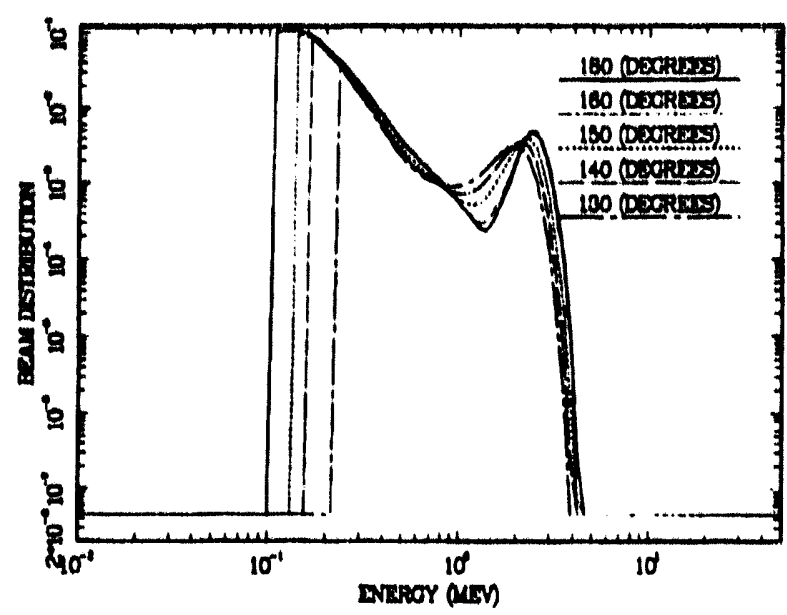

Figure 13d. $\mathrm{t}=20.0 \mathrm{~ns}$.

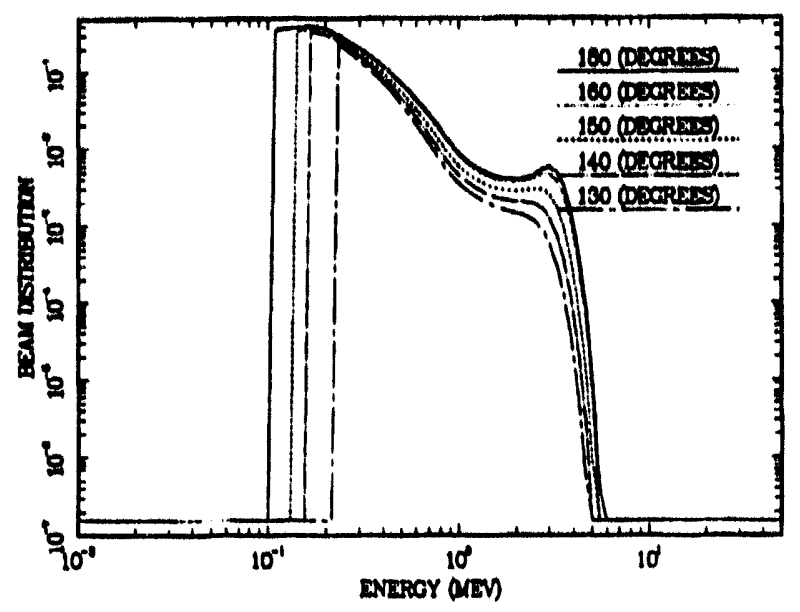

Figure 13e. $\mathrm{t}=50.0 \mathrm{~ns}$.

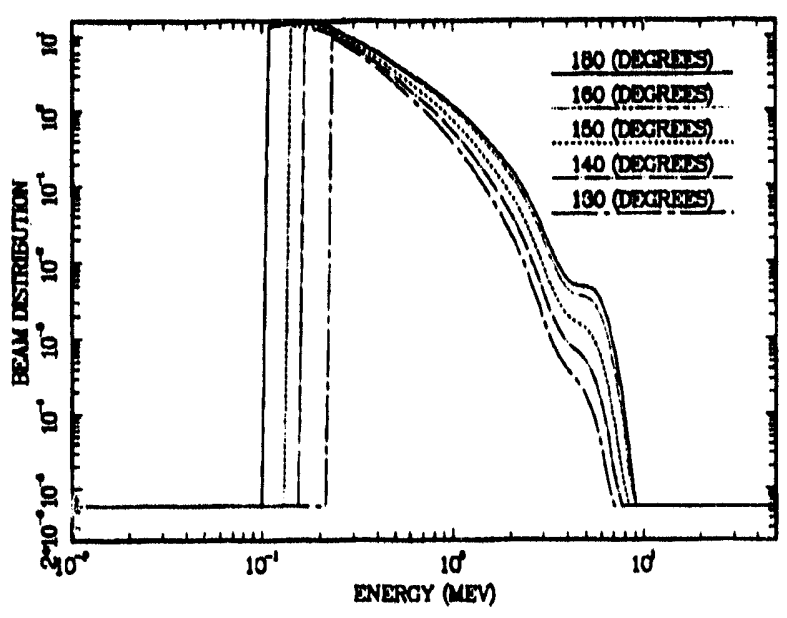

Figure 13f. $t=150.0 \mathrm{~ns}$.

Figure 13. Time history of beam distribution function. 


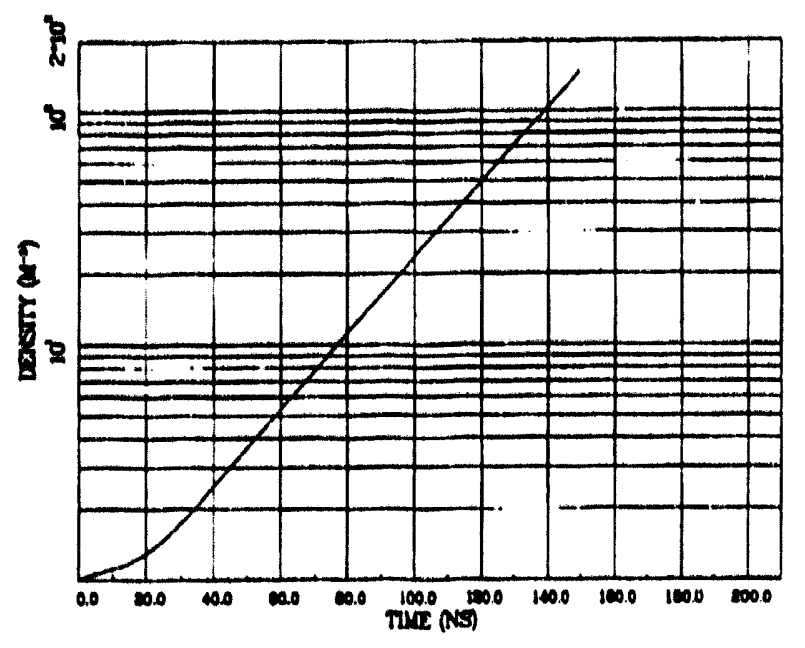

Figure 14a. Electron density.

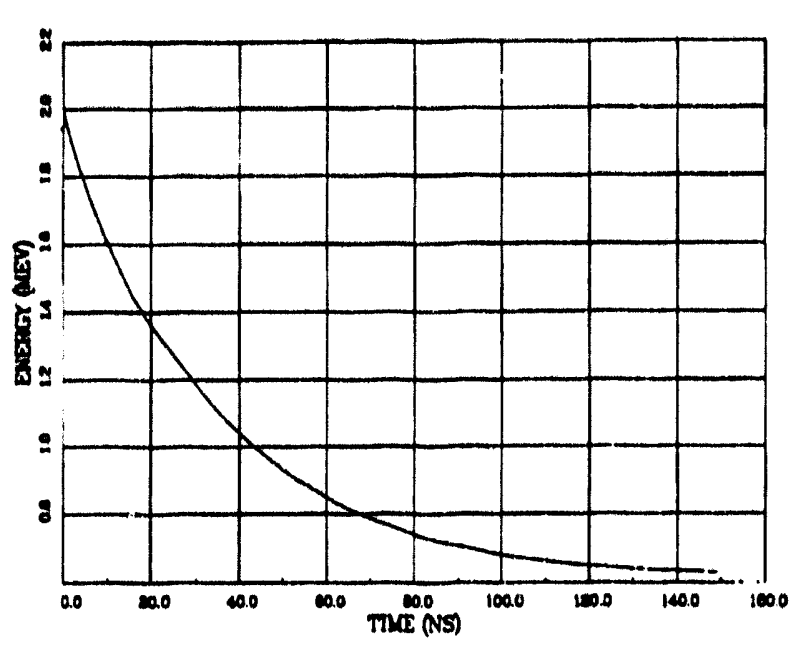

Figure 14b. Average kinetic energy.

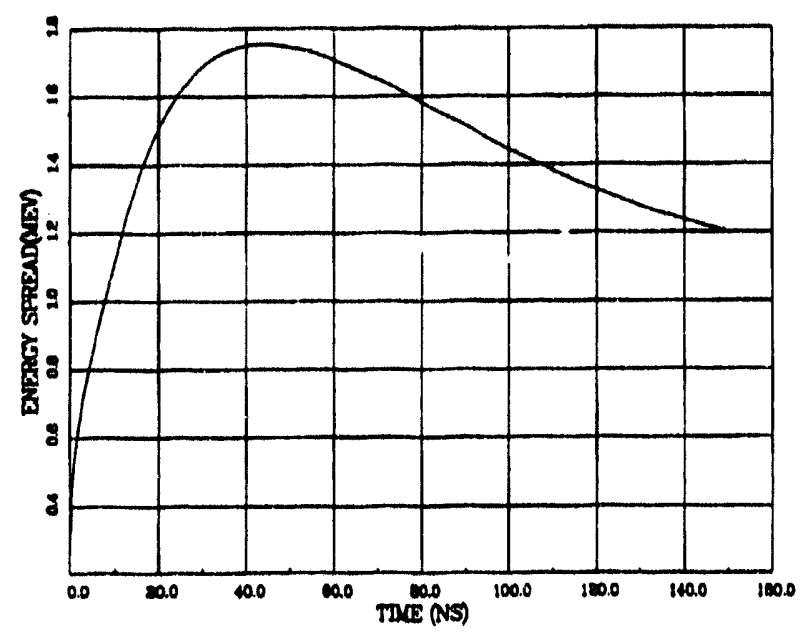

Figure 14c. Kinetic energy spread.

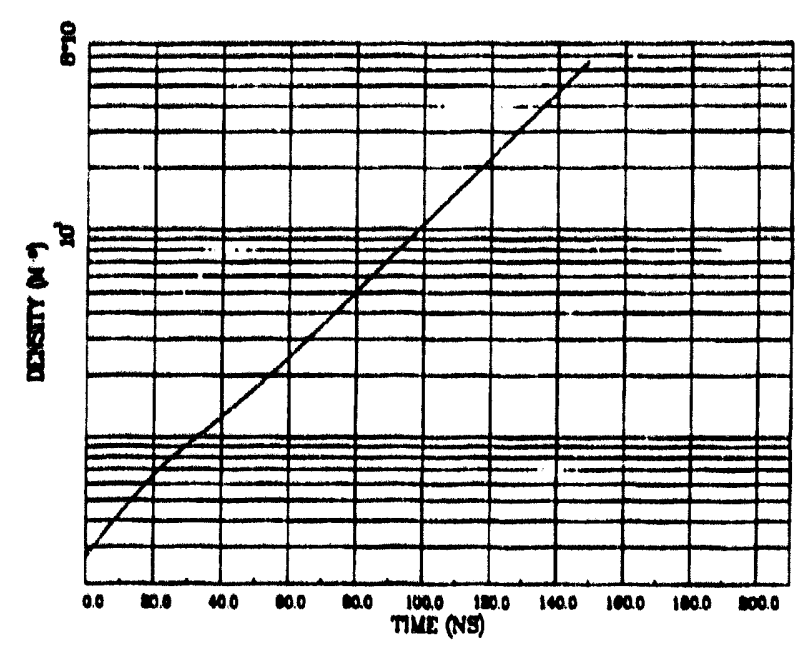

Figure 14d. Beam density.

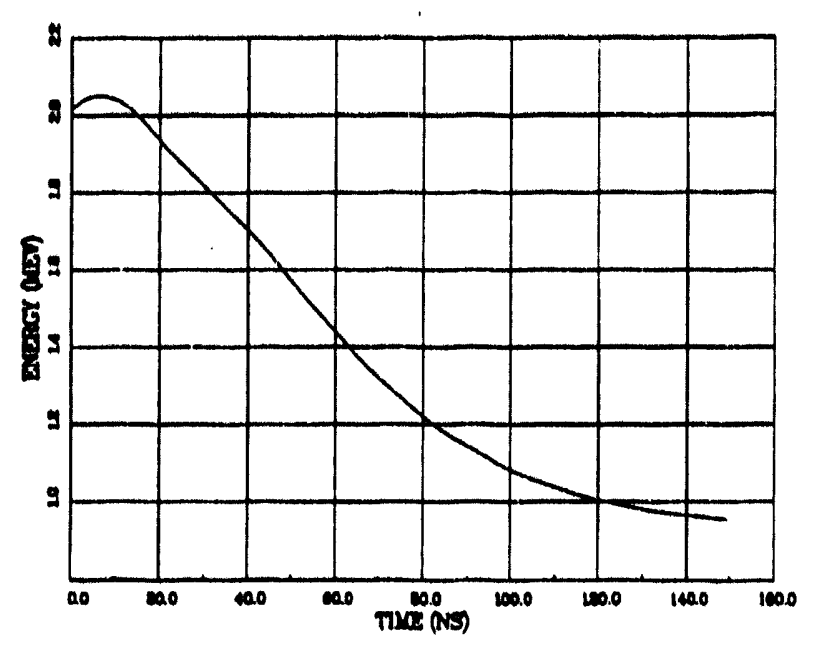

Figure 14e. Beam average kinetic energy.

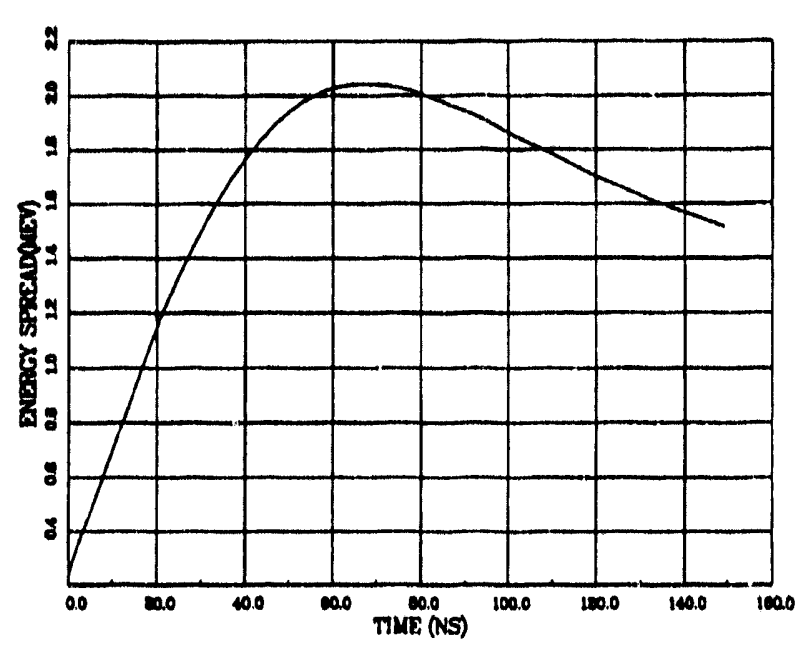

Figure 14f. Beam kinetic energy spread.

Figure 14. Moments of the overall and beam distribution function. 


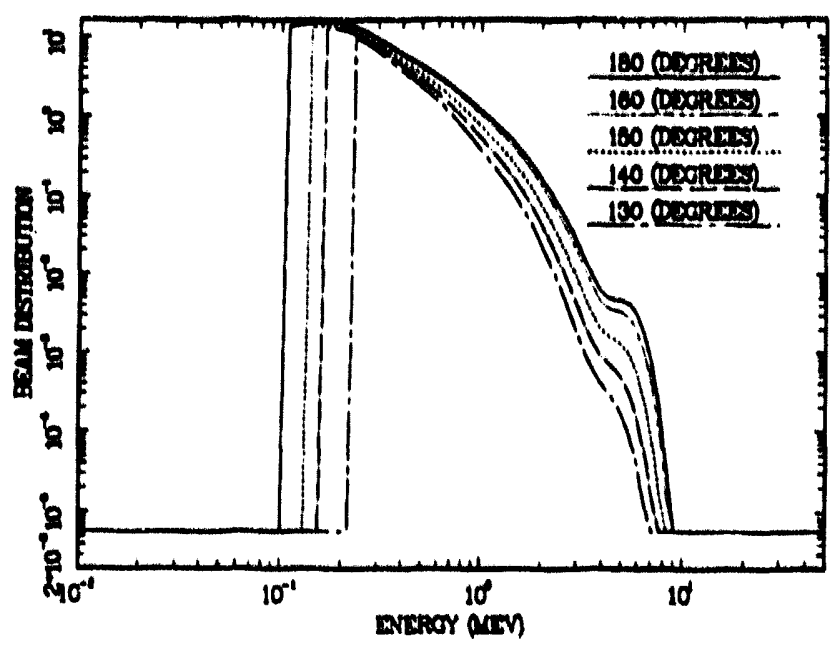

Figure 15a. $\delta_{0}=2.0$.

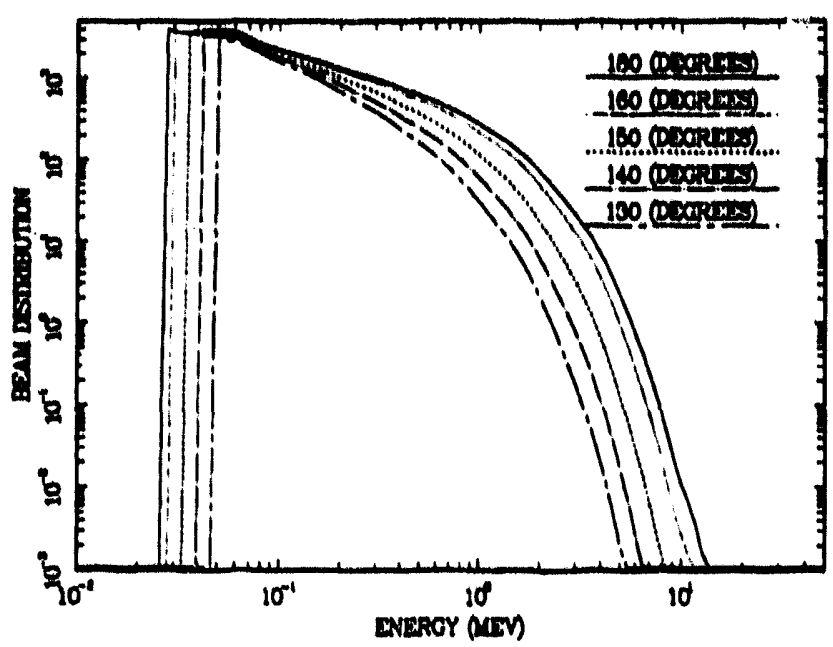

Figure $15 \mathrm{~b} . \delta_{0}=5.0$.

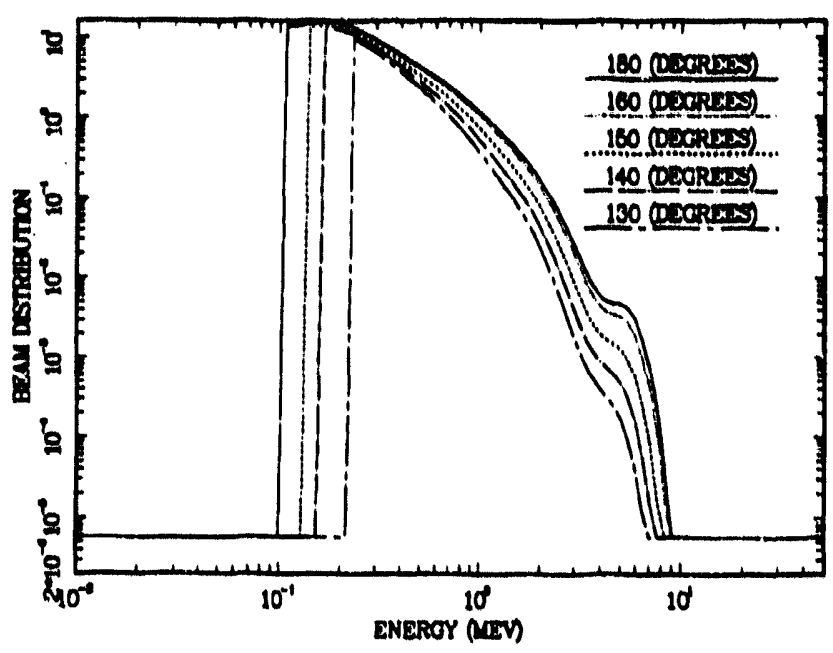

Figure $15 \mathrm{c} . \delta_{0}=8.0$.

Figure 15. Self-similar beam distribution function. 


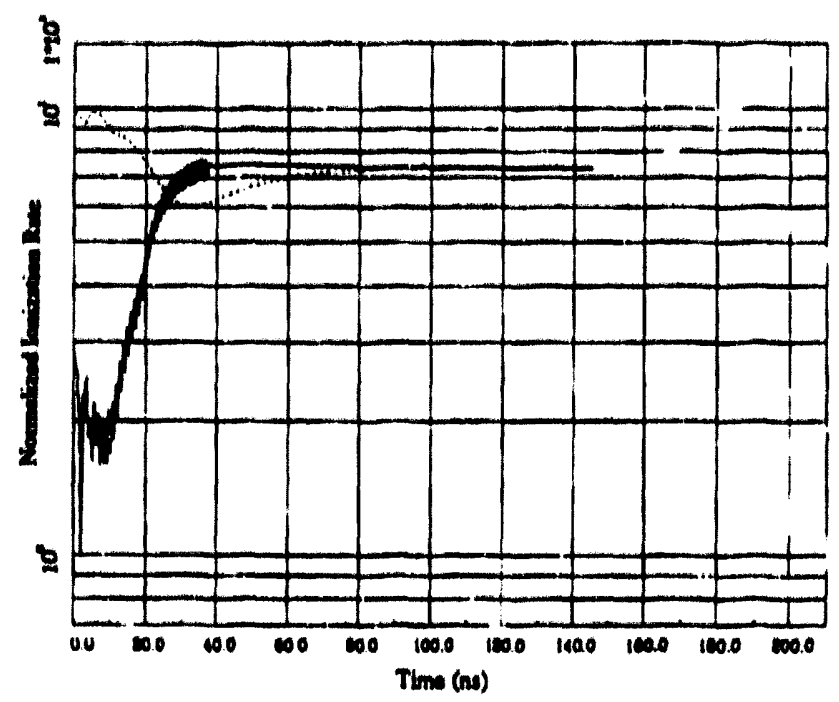

Figure 16a. Ratio of ionization rates for $\delta_{0}=2.0$.

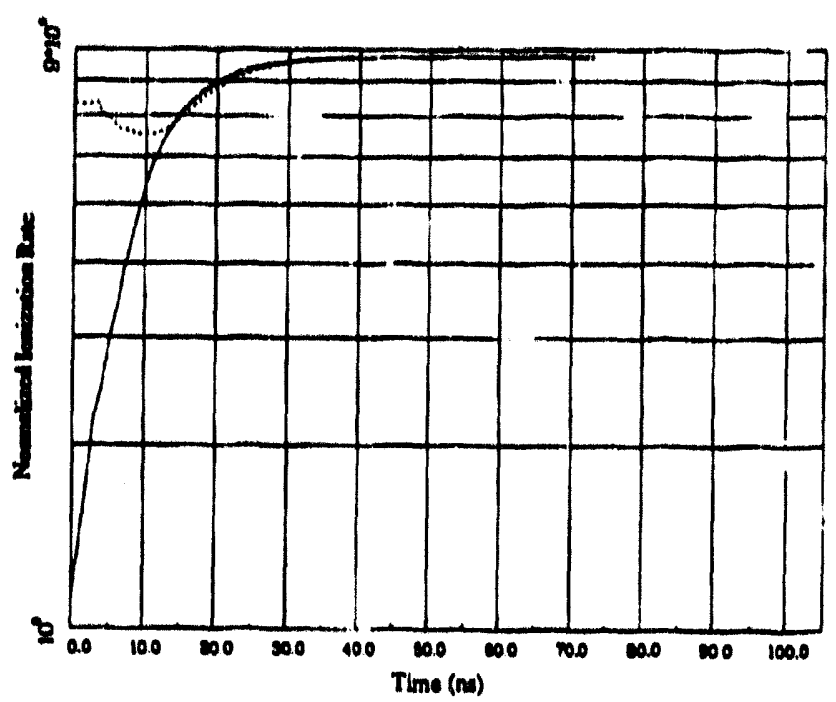

Figure 16b. Ratio of ionization rates for $\delta_{0}=5.0$.

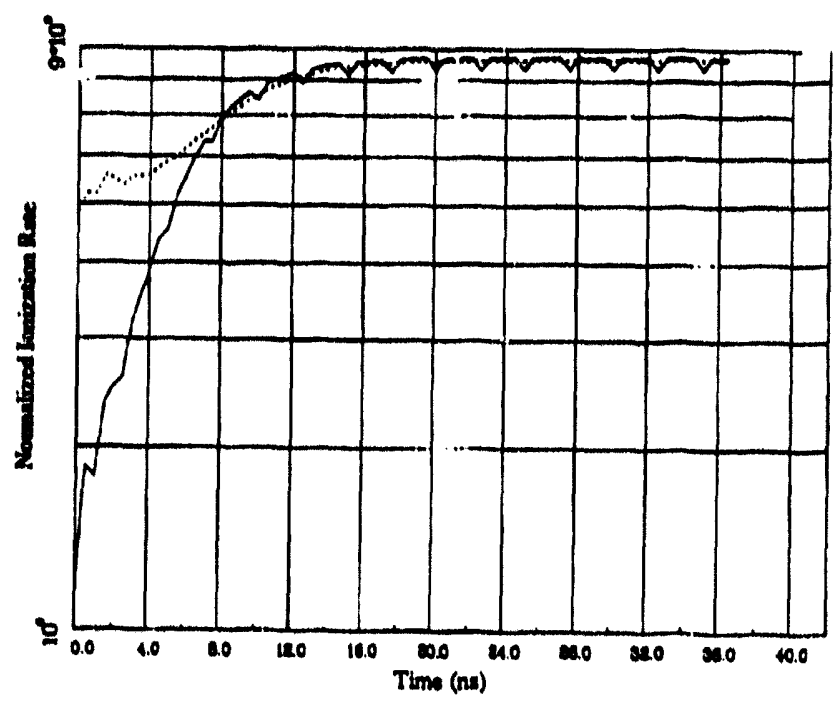

Figure 16c. Ratio of ionization rates for $\delta_{0}=8.0$.

Figure 16. Ratio of ionization rates. 


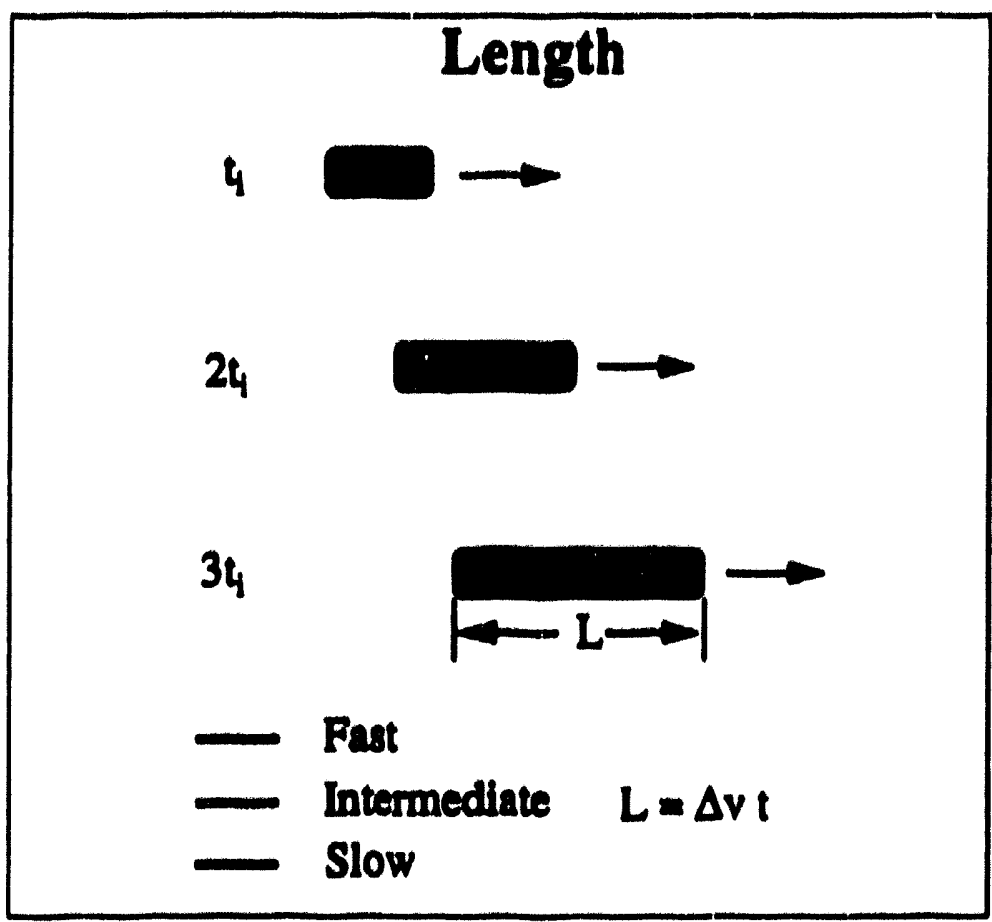

Figure 17a. Evolution of beam length.

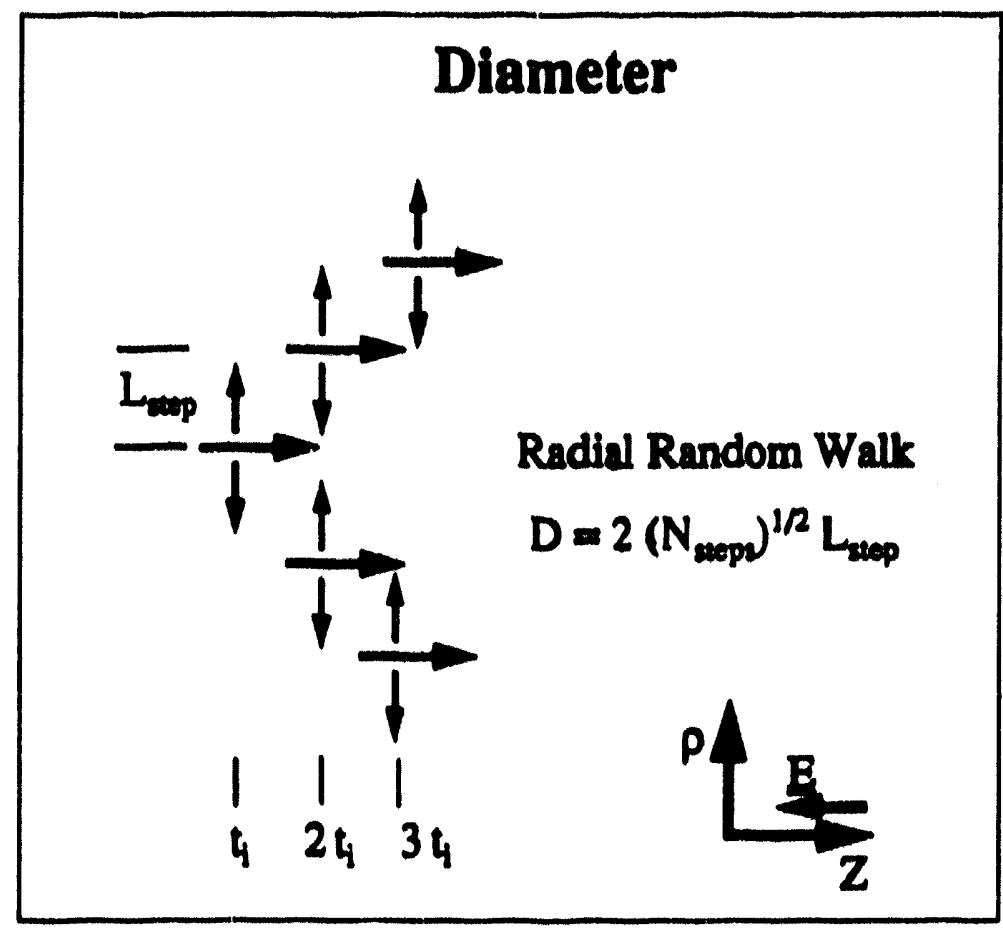

Figure 17b. Evolution of beam diameter.

Figure 17. Spatial dimension of electron beam. 


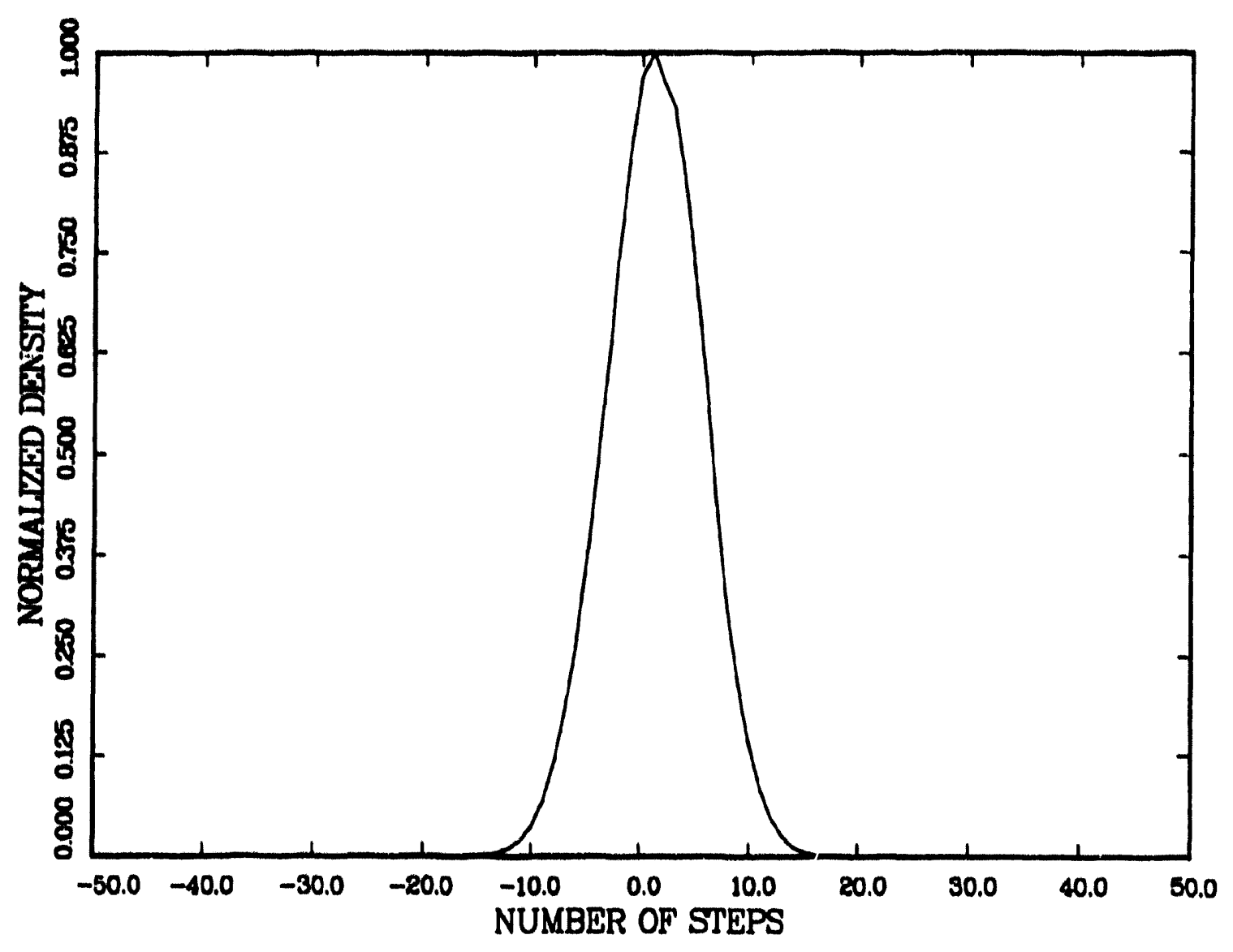

Figure 18. Beam profile after 40 e-foldings. 


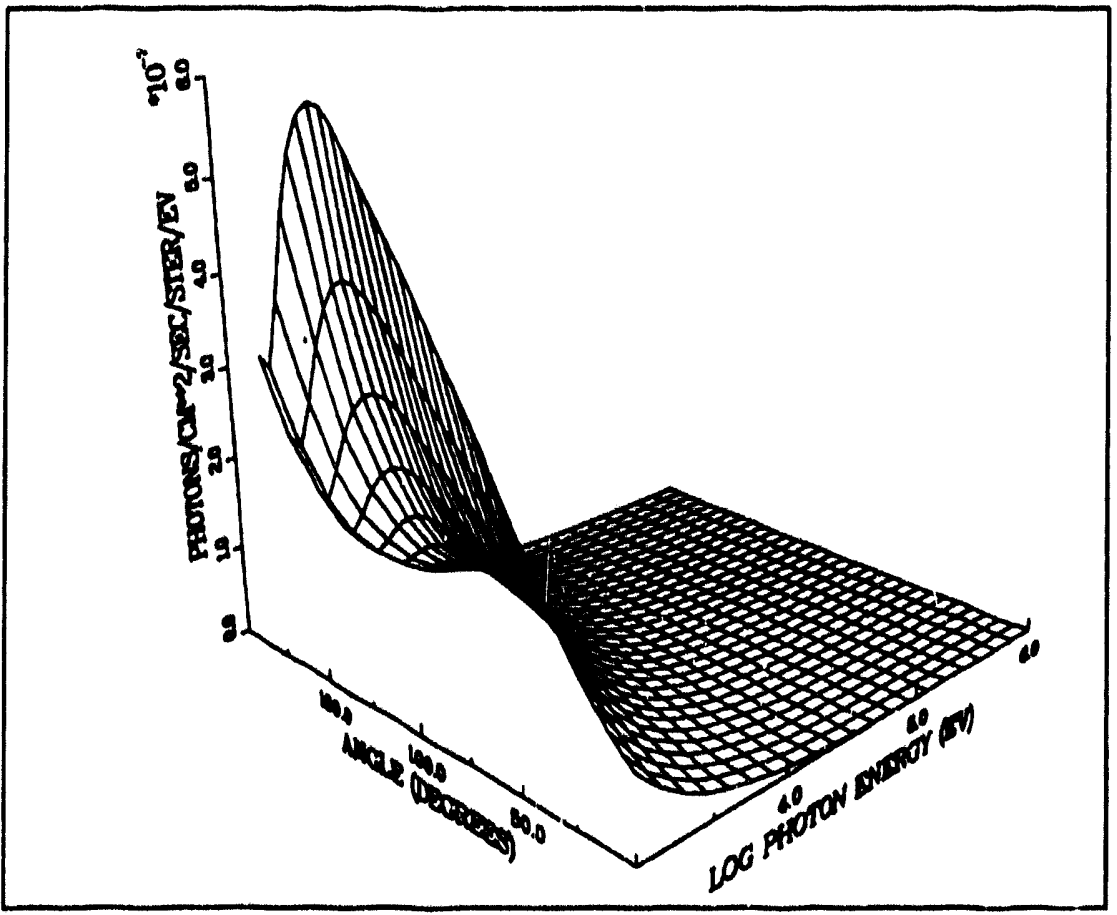

Figure 19a. Three-dimensional plot.

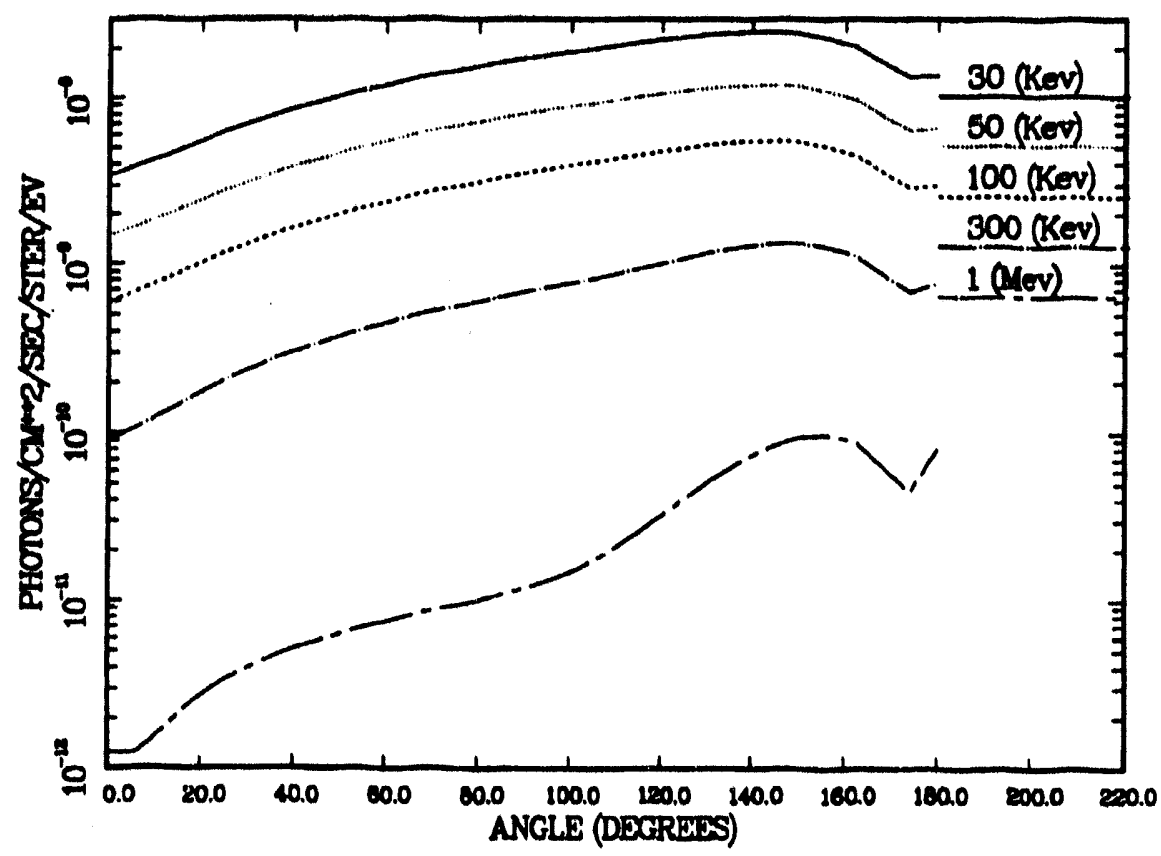

Figure 19b. Two-dimensional plot for various primary energies.

Figure 19. Bremsstrahlung emission. 


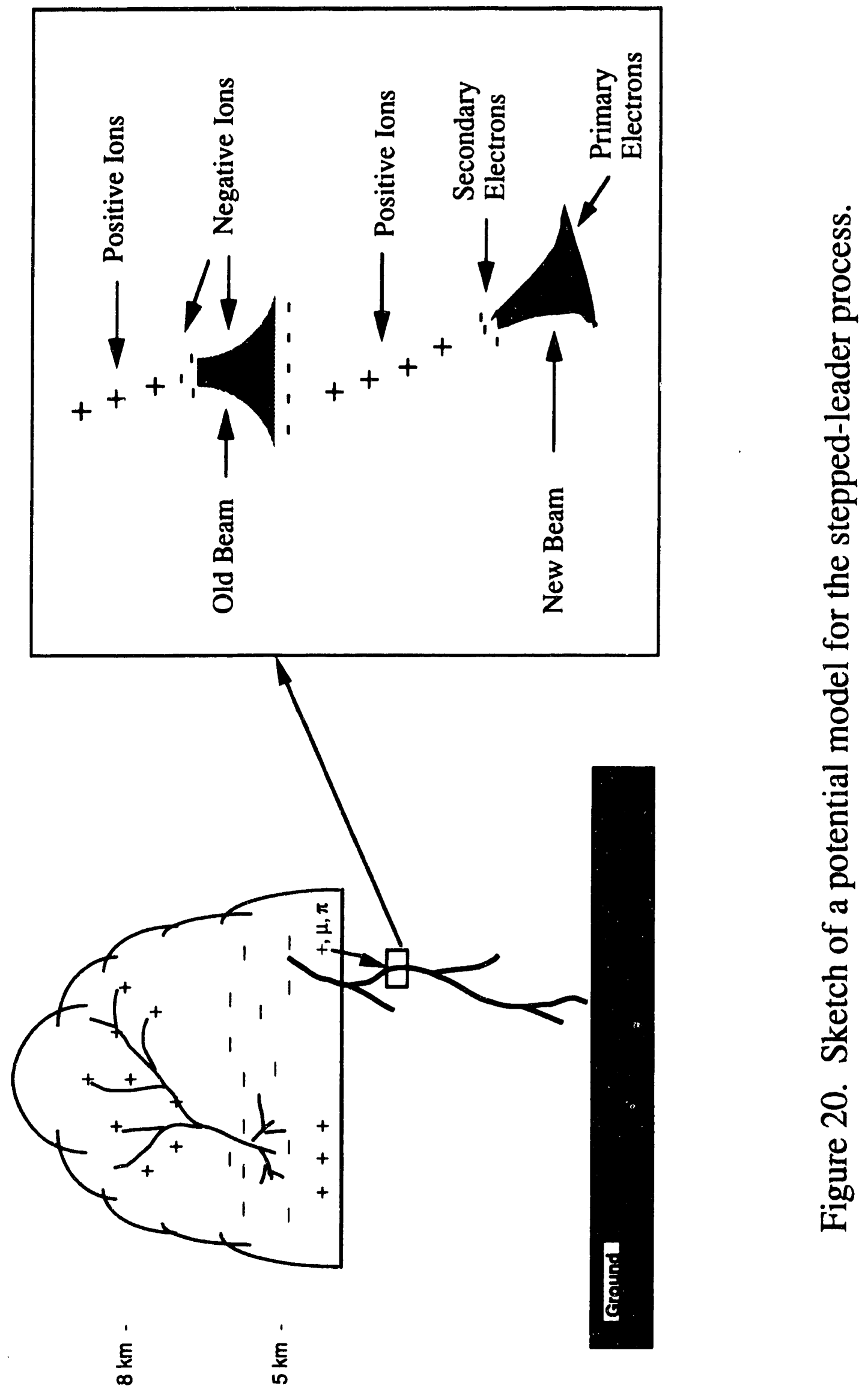



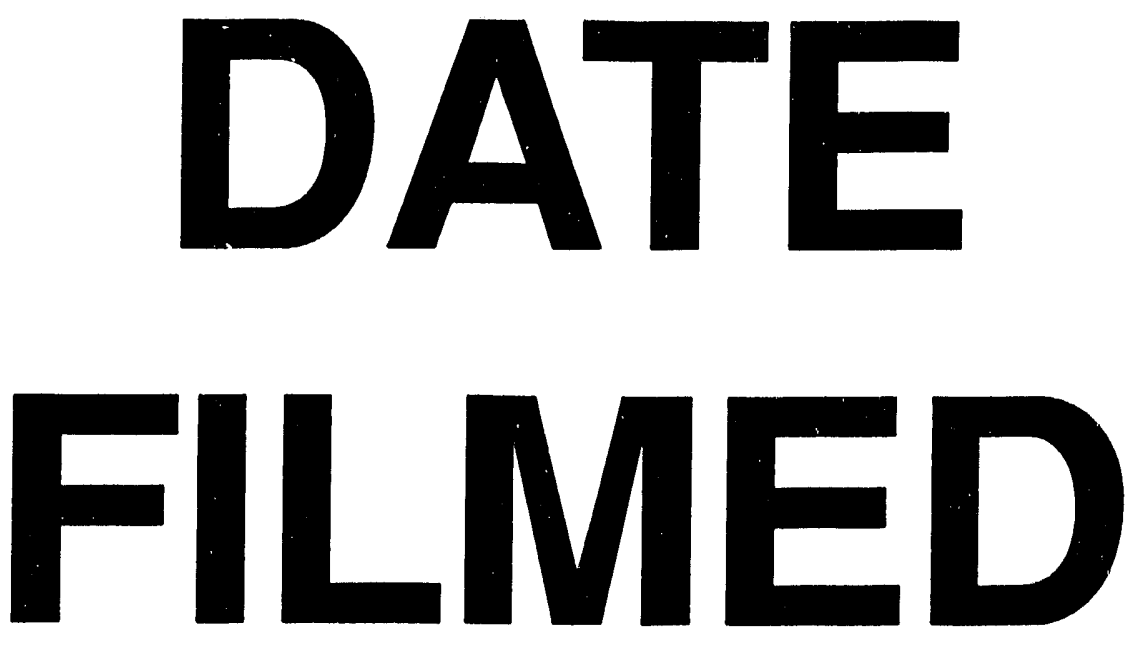

$6 / 9 / 94$
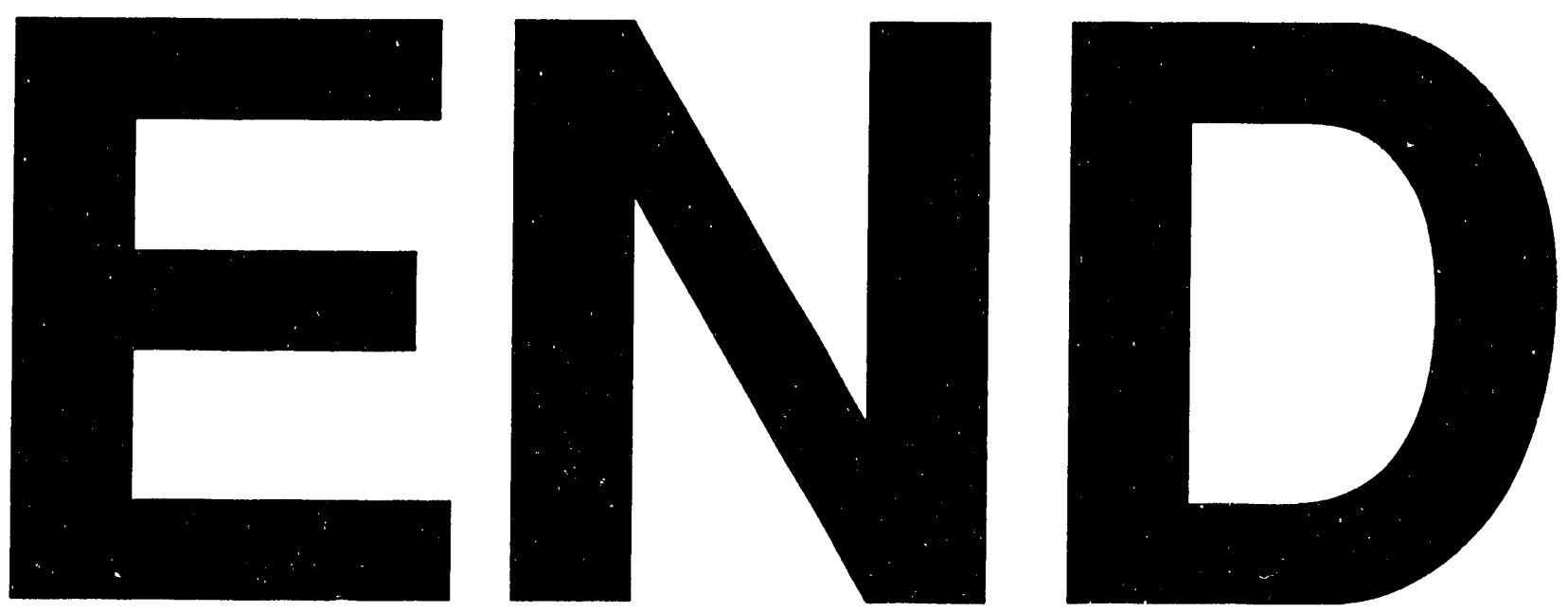